\title{
THEORY OF s-d EXCHANGE SCATTERING IN DILUTE MAGNETIC ALLOYS
}

Thesis By

Anupam Madhukar

In Partial Fulfillment of the Requirements for the Degree of Doctor of Philosophy

California Institute of Technology Pasadena, California 
To my Professor 


\section{ACKNOWLEDGMENT}

The author wishes to express his sincere appreciation for the continued helpful and encouraging support provided by Dr. C. C. Tsuei throughout this work. It is a pleasure to acknowledge the stimulating discussions with my friends Dr. U. Trivedi, Dr. N. Patel and Mr. A. Thyagaraja. Thanks are extended to Professor R. P. Feynman for two very encouraging discussions at the early stages of this work, and to Professor R. L. Mo'ssbauer for discussing the results and bringing certain experimental results to the authors knowledge. A very helpful correspondence with Professor R. D. Mattuck is gratefully acknowledged.

The computer work done by Mr. Yoshiaki Daimon is greatly appreciated. Special thanks are due to Mrs. Betty Wolar, not only for numerous typing jobs, but also for her friendship which has in many ways been responsible for this thesis.

Last, but by no means least, it is a privilege to acknowledge the guidance and infinite patience of my advisor Professor Pol Duwez, to whom this the sis is dedicated.

Financial aid provided by the Atomic Energy Commission and California Institute of Technology is very much appreciated. 


\section{ABSTRACT}

The problem of $s-d$ exchange scattering of conduction electrons off localized magnetic moments in dilute magnetic alloys is considered employing formal methods of quantum field theoretical scattering. It is shown that such a treatment not only allows for the first time, the inclusion of multiparticle intermediate states in single particle scattering equations but also results in extremely simple and straight forward mathematical analysis. These equations are proved to be exact in the thermodynamic limit. A self-consistent integral equation for electron self energy is derived and approximately solved. The ground state and physical parameters of dilute magnetic alloys are discussed in terms of the theoretical results. Within the approximation of single particle intermediate states our results reduce to earlier versions. The following additional features are found as a consequence of the inclusion of multiparticle intermediate states;

(i) A non analytic binding energy is present for both, antiferromagnetic $(\mathrm{J}<0)$ and ferromagnetic $(\mathrm{J}>0)$ coupling $s$ of the electron plus impurity system.

(ii) The correct behavior of the energy difference of the conduction electron plus impurity system and the free electron system is found which is free of unphysical singularities present in earlier versions of the theories. 
(iii) The ground state of the conduction electron plus impurity system is shown to be a many-body condensate state for $\mathrm{J}<\mathrm{O}$ and $\mathrm{J}>0$, both. However, a distinction is made between the usual terminology of "Singlet" and "Triplet" ground states and nature of our ground state.

(iv) It is shown that a long range ordering, leading to an ordering of the magnetic moments can result from a contact interaction such as the s-d exchange interaction.

(v) The explicit dependence of the excess specific heat of the Kondo systems is obtained and found to be linear in temperatures as $T \rightarrow 0$ and $T \ln T$ for $0.3 T_{K} \leq T \leq 0.6 T_{K}$. A rise in $(\Delta C / T)$ for temperatures in the region $0<\mathrm{T} \leq 0.1 \mathrm{~T}_{\mathrm{K}}$ is predicted. These results are found to be in excellent agreement with experiments.

(vi) The existence of a critical temperature for Ferromagnetic coupling $(\mathrm{J}>0$ ) is shown. On the basis of this the apparent contradiction of the simultaneous existence of giant moments and Kondo effect is resolved. 
vi

TABLE OF CONTENTS

PAR T

TITLE

PAGE

I. INTRODUCTION

II. FORMATION OF LOCALIZED MAGNETIC

MOMENT: ANDERSON MODEL

III. s-d EXCHANGE MODEL

IV. FORMULATION OF THE THEORETICAL PROBLEM

A. Field Theoretic Scattering Equations

B. Notation for Many Electrons Plus Impurity Wave Functions

C. The Exact Single Particle Scattering Equations

v. SOLUTION OF THE SCATTERING EQUATIONS

A. Solution in the Single Particle Intermediate State Approximation

B. Contribution of Multipa rticle Intermediate States

C. Solution of Exact Single Particle Scattering Equations

D. Self Consistent Equation for Self Energy

E. Some Mathematical Properties of the Functions Involved

VI. EVALUATION OF SELF ENERGY

A. Approximate Evaluation and Comparison with Previous Results

B. Numerical Analysis

VII. ON DENSITY OF STATES

VUI. INTERPRETATION OF RESULTS 
vii

Table of Contents (Cont'd)

PAR T

PAGE

B. The Ground State

C. Negative $\mathrm{J}$ vs Positive $\mathrm{J}$ : Resolution of an Apparent Contradiction

IX. ON PHYSICAL PARAMETERS

A. Resistivity and Thermoelectric Power

B. Specific Heat

APPENDIX

163

REFERENCES

171 


\section{INTRODUCTION}

The work reported in the following pages has been entitled "Theory of s-d Exchange Scattering in Dilute Magnetic Alloys." True to the traditions of theoretical work, it is an attempt to solve a somewhat idealized model, the so called s-d Exchange model, built to represent a very complex realistic system of a magnetic impurity placed in a non-magnetic host metal and their consequent interaction. The term "Magnetic Alloy" presupposes the existence of the magnetic moment of the impurity atom even under the influence of the non-magnetic environment of the host metal. Nowhere in the present work therefore, has the very important question of the existence of the magnetic moment of the impurity atom been considered. However, for completeness sake, a few words on this question are not only in order, but most necessary to bring about the actual physics of the situation. Let us therefore consider the following situation; supposing we were to take a single free atom which has a magnetic moment e.g. $\mathrm{Fe}, \mathrm{C}_{\mathbf{r}}$, Ni etc. and put it at one of the lattice sites of a non-magnetic host metal e.g. Cu. The resuiting disturbance can significantly affect the characteristics of the impurity atom and the host as well. The two basic questions with which the field of impurity magnetism is concerned are (1) Under what conditions would the impurity atom, which had the property of being magnetic in its free state, retain its magnetic moment even under the influence of the environment of the non-magnetic host metal? (2) Provided the impurity atom retains its magnetic moment, 
what are the properties of the resulting system of magnetic impurity and the host metal.

The investigation of the criteria for the impurity atom to retain its magnetic moment starts with the pioneering work of Friedel. (1) Anderson ${ }^{(2)}$ and Wolff ${ }^{(3)}$ proposed models for more quantitative and rigorous discussions of the criteria within the frame work of Hartree-Fock approximation. We shall not go into the details of these works here, as the present work is concerned with the second question raised earlier, namely the effect of the magnetic moment and the nature of the resulting magnetic alloy. These aspects we shall consider on the basis of the $s-d$ exchange model, which finds its justification as a reasonable theoretical model describing the interacting system of the localized magnetic moment and conduction electrons of the host by virtue of the fact that it has been shown to be the limit of the Anderson Model when a localized magnetic moment exists in the system. Because of the central role played by the Anderson Model ${ }^{(4)}$ in the theory of impurity magnetism, we shall present a brief discussion of its physical origin and its relationship with the s-d exchange model in Section II. This shall also serve to clarify the relationship of the s-d exchange model with the realistic system of magnetic moments in non-magnetic host metals and alloys.

Coming to the question of the effect of the magnetic moment on the properties of the host, we find that the introduction of a very small concentration of magnetic impurities can change the electronic 
properties of the metal in a very distinct manner. (See fig. 1) For example in systems like transition metal impurities such as $\mathrm{Cr}, \mathrm{Mn}, \mathrm{Fe}$ in noble metals $\mathrm{Cu}, \mathrm{Ag}$ and $\mathrm{Au}$, one observes a minimum in the electrical resistivity which depends on the impurity concentration, a peak in the specific heat, deviations from Curie and Curie-Weiss behavior in the low temperature susceptibility, a giant thermo-electric power and a strong dependence of the resistivity on a, longitudinal or transverse, magnetic field. Similarly, anomalies are observed in the line width of NMR and M 8 ssbauer spectra. The details of these experimental works can be found in the review articles by Vandenberg, (6) Daybell and Steyert, ${ }^{(7)}$ and Heeger. (8)

In an attempt to explain the se anomalous properties, Kondo (9) in 1964 calculated, to second Born approximation, the scattering amplitude of the conduction electrons off localized magnetic moments, based on the s-d exchange model, and was successful in explaining the minimum in resistivity as a function of temperature. However, his calculation posed a very interesting problem for the theorists, for it showed that the scattering amplitude of an electron at the Fermi energy diverges logarithmically at absolute zero of temperature, when the statistics of the conduction electrons is duly taken care of. Later perturbational calculations have shown that more generally, no matter how small the exchange interaction $\mathrm{J}$ is, perturbation theory breaks down due to the persistence of intrinsically divergent terms of the form $(J \rho)^{n}\left\{\ln k \frac{k}{6} / \epsilon_{F}\right\}^{m} m=0,1, \ldots(n-1)$. This has 


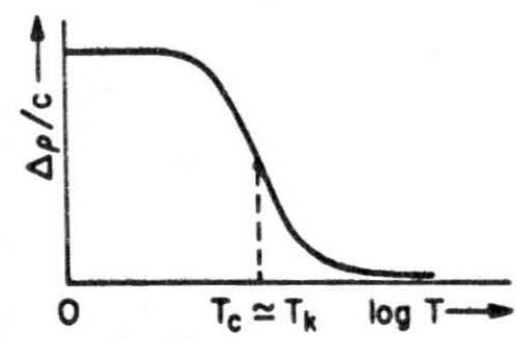

(a)

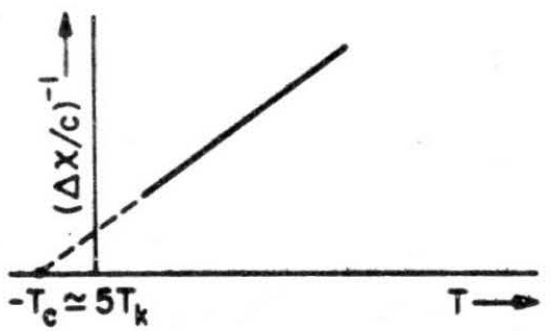

(c)

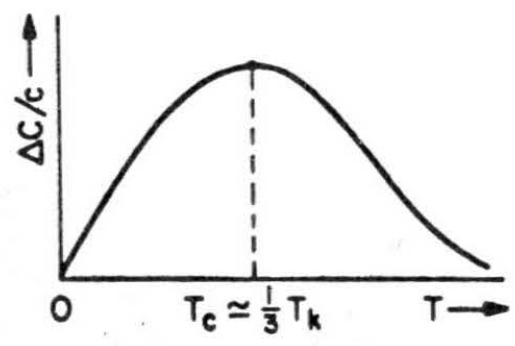

(b)

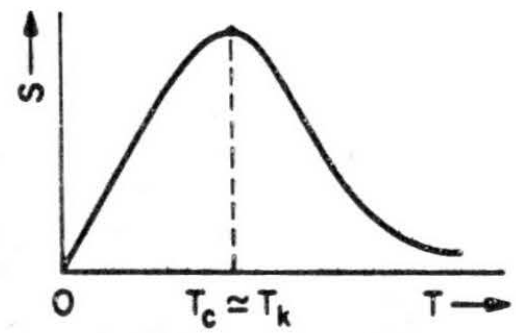

(d)

Fig. I Qualitative behavior of the anomalous contribution per at $\%$ to (a) Resistivity $(\Delta P / c)$ (b) Specific Heat $(\Delta C / c)$ (c) Magnetic Susceptibility $(\Delta X / c)$ (d) Thermoelectronic Power (S) 
come to be known as the "Kondo Problem."

The Kondo effect, or logarithmic divergence in the scattering amplitude arises from the fact that for spin dependent scattering the statistical factors in the intermediate states for the particle and hole scattering do not cancel each other. The impurity spin represents an internal degree of freedom and can change during the scattering process, making it dependent upon the order in which the system assumes different intermediate states. This order is different for electrons and holes. At $T=0$ a non-cancelled fermi factor restricts the intermediate state sums to energies smaller than the Fermi energy $E=0$. The sum over $E(q)$ contains the factor $\left(E-E_{q}\right)^{-1}$ and thus becomes proportional to lnE. Thus the scattering amplitude for an incoming electron with fermi energy diverges at $\mathrm{T}=0$. This divergence is no longer present at finite temperatures where the Fermi function is no longer a step function. The effect of the Fermi statistics on a single scattering process indicates that the anomalies observed in dilute magnetic alloys cannot be explained within the frame work of one electron theory as they are indeed true many body properties. Physically, the true nature of the many body phenomenon may be looked upon as follows; the scattering of an electron depends on the spin state in which the impurity atom is. The state of the impurity atom however, depends upon the scattering process of an earlier electron. In other words, the scattering of an electron depends upon the state in which the impurity was left by its predecessor. The scattering 
of this predecessor in turn depends upon earlier scattering process. The impurity thus correlates different scattering process and generates an indirect interaction between the conduction electrons. This is reminiscent of the situation in Superconductivity where electron-electron interactions are generated via virtual phonons.

Thus it should be clear that the apparent simplicity of the seemingly innocent looking $s-d$ exchange interaction is extremely deceptive. It's theoretical solution requires full powers of many body theory available to us today, and that too, in a non-perturbative way. The work reported in this thesis is an attempt in this direction. However, the approach being significantly different from any other employed to investigate this problem (and most probably different also from the approaches taken in general in many body the ory). we are forced by our obligation to history to say something on the other approaches to this problem.

Since Kondo's original paper, almost all the well known techniques of many body theory have been used by various people in the field with different measures of success. Nagaoka ${ }^{(10)}$ introduced Zubarev's ${ }^{(11)}$ double time Green's function technique of statistical physics to derive the equations of motion of the conduction electron. Since then many people have taken this approach, sometimes with minor modifications, but perhaps the solutions of Bloomfield and Hamman ${ }^{(12)}$ and Zittarz and Mueller-Hartmann (13) provide the most reasonable expression for resistivity and specific heat, of 
use to the experimentalist. On the other hand Suhl ${ }^{(14)}$ borrowed the Chew-Low formalism of high energy scattering from elementary particle physics and provided us with two coupled non-linear integral equations for spin flip and spin non-flip scattering amplitudes, in the approximation of single particle intermediate states. The most basic assumption in this approach is the one to one correspondence between the eigen functions of the unperturbed and total Hamiltonians. Suhl and Wong, ${ }^{(15)}$ Kondo ${ }^{(16)}$ and others have solved the se coupled integral equations using dispersion theoretic techniques. It is found that the Kondo singularity in the scattering amplitude is replaced by a resonance pole occurring at a temperature, known as the Kondo temperature, given by

$$
T_{K}=C \quad e^{\frac{1}{T T_{0}} \rho\left(E_{F}\right)}
$$

Thus it is seen once again that $T_{K}$ being non-analytic as a function of the exchange interaction strength $\mathrm{J}$, ordinary perturbation theory of quantum mechanics cannot provide us with meaningful solutions. It is the assertion of Suhl's theory that the true ground state at absolute zero is reached by a proper analytic continuation for $T<\mathrm{T}_{\mathrm{K}^{*}}$ The very powerful and perhaps the most popular of many body techniques, namely summation of infinite number of Feynman diagrams of a given order has also been used by many. Suhl's scattering equations have been rederived using this method and are shown to be correct only to logarithmic accuracy. (17) Brenig and Gbtze (18) have attempted to improve on this by considering next higher order dia- 
grams, which in the language of scattering theory amounts to the inclusion of some three particle i. e. a particle plus a particle hole pair states in the intermediate states. Besides the conventional Feynman diagram technique, Abrikosov ${ }^{(19)}$ and Doniach ${ }^{(20)}$ have used their own version of the diagram technique, but there is no improvement upon the results obtained by the previously mentioned techniques.

In addition to the above mentioned three most commonly employed techniques, the variational technique has also been used by Kondo and Applebaum ${ }^{(21)}$ and by Yosida, (22) the latter being essentially based on the idea of Cooper's Model in Supe rconductivity. (23) Cooper type of model for s-d exchange scattering was proposed by Kondo. (24) On the other hand, Takano and Ogawa ${ }^{(25)}$ proposed a decoupling scheme in Nagaoka's equation of motion method corresponding to Gorkov's ${ }^{(26)}$ in the case of superconductivity.

The upshot of these various attempts to solve the problem is some approximate results for physical parameters and thermodynamic quantities. However, all these results being correct only to the leading order singularity, namely logarithmic, they fail to provide any theoretical understanding of the situation near the Kondo temperature, which happens to be the most important region. For example, within the existing theoretical solutions, the energy difference between the ground state of the total Hamiltonian and the unperturbed Hamiltonian is given by the solid lines of Fig. (2). The dashed line is the conjectured result. The nature of the ground state of the system of impurity and host metal is still an open question. The 
opinion of the people may be divided into two groups. One group feels that the actual ground state of the system is foreign to any kind of perturbational treatment and as such, cannot be realized by such approaches. The other believes that the analytic continuation of the perturbational result for $T>T_{K}$ to regions of $T<T_{K}$ is sufficient to obtain the actual ground state. However, one notices that the actual ground state energy, within all such approaches, has a part which is non-analytic in the exchange interaction $\mathrm{J}$. This has been associated with the concept of som e kind of binding energy associated with the actual ground state. Thus, whether the actual ground state corresponds to the ground state of "normal" system or is something totally different, is still an open question.

It has become very well known now that spin life time effects play an important $r$ ole at very low temperatures. In the language of scattering theory, this means that below $T_{K}$, the multiparticle intermediate states become important. Since the existing results are correct at most within single particle intermediate states, it is not surprising that they fail to provide reasonable expressions for physical parameters at very low temperatures and that their prediction of the ground state is doubtful.

To conclude this section on existing theories we summarize the situation as follows: Although Suhl, Nagaoka or BloomfieldHamman theories ${ }^{(5)}$ correctly predict the leading logarithmic terms, it is definitely not clear how far they are correct with regard to the less divergent terms, as has been noted by Silverstein and Duke. (17) 
The leading terms alone do not uniquely determine the low temperature properties when continued across $T_{K^{*}}$ On the contrary, the less divergent terms are as important as the former and determine all the physics below $\mathrm{T}_{\mathrm{K}^{*}}$ 


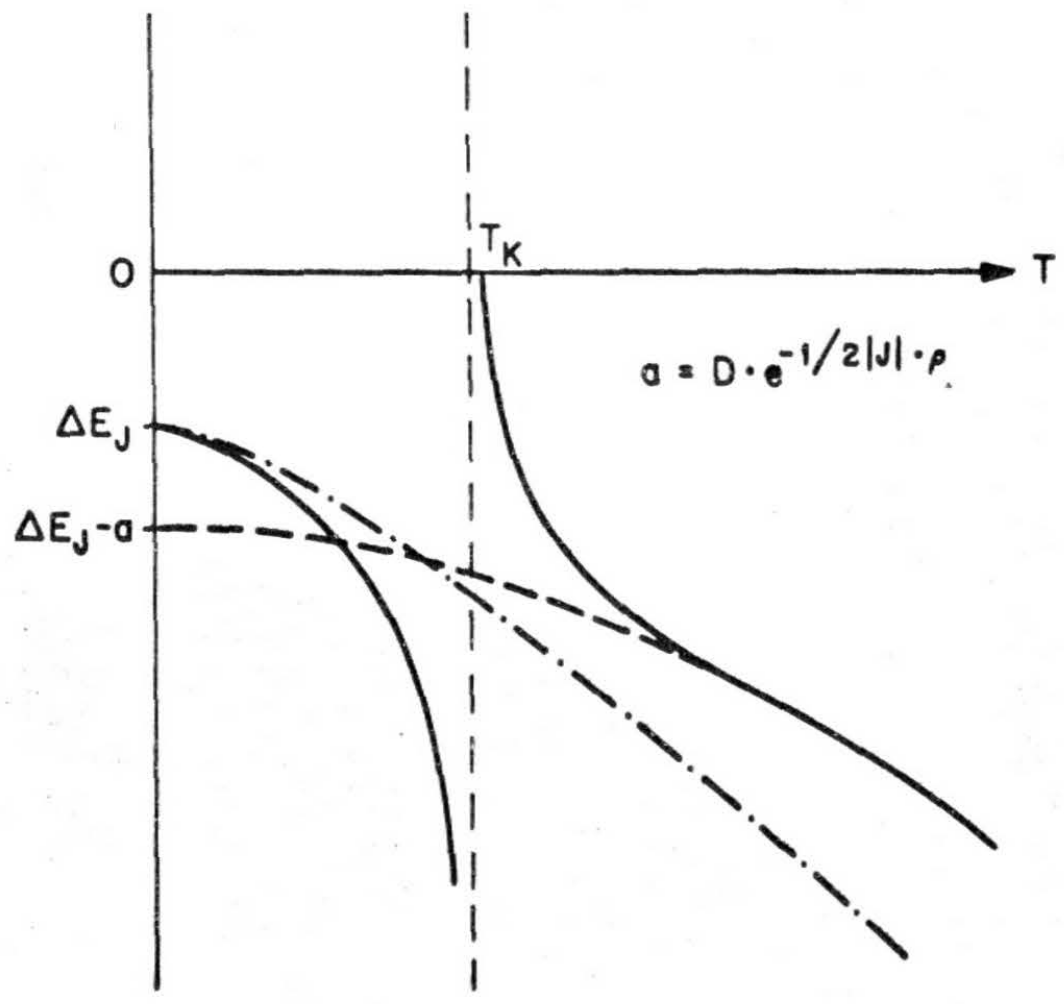

Fig. 2. Energy shift (or Free Energy shift) as a function of temperature. Solid line represents the case $\mathrm{J}<0$. Dashed curve represents the conjectured behaviour for $\mathrm{J}<0$, going to $\left(\Delta \mathrm{E}_{J^{-a}}\right)$. The dash-dot curve represents the case $J>0 . \quad \Delta E_{J}$ is the perturbation theory result for energy shift. 
II. FORMATION OF LOCALIZED MAGNETIC MOMENTS:

\section{ANDERSON MODEL}

The Anderson model ${ }^{(2)}$ is an attempt to provide a simplified picture of handling atomic physics in metallic environment. The model approximates this complex system by the following Hamiltonian,

$$
H=H_{s}+H_{d}+H_{s-\alpha}
$$

Here $\mathrm{H}_{\mathrm{s}}$ is the Hamiltonian giving the energy levels of the host metal;

$$
H_{s}=\sum_{k r} \epsilon_{k} a_{k r}^{+} a_{k r}
$$

where $\epsilon_{K}^{\prime}$ s are the single electron energies and $a_{k r}^{+}, a_{k \sigma}$ the usual creation and anhilation operators. This very simple one-electron Hamiltonian is surely a gross over simplification to a real metal, in which electron-electron, electron-phonon etc. interactions play a crucial role. The se many body terms are neglected for simplicity and because such a neariy free electron model finds formal justification in the Landau theory of Fermi liquids. 
The term $\mathrm{H}_{\mathrm{d}}$ stand $\mathrm{s}$ for the atomic physics of the impurity atom. It is clear from the outset that if one is interested in impurity magnetism on must keep the electron-electron interactions explicitly in the problem for it is such terms which cause atomic magnetism in the transition series. This is brought about in two ways. a). The direct Coulomb interaction $U$ between two electrons on outer shell of an atom is of order $\left(\mathrm{e}^{2} / \mathrm{a}_{0}\right)$ where $a_{0}$ is the Bohr radius. This large value ( $\sim 20 \mathrm{eV}$ ) serves to keep the neutral configuration of the atom stable i.e., the number of electrons equal to the protons. This is non-trivial for it allows the atom to have an odd number of electrons and hence causes atomic magnetism (without the Coulomb interaction all levels would be doubly occupied and hence no magnetism). b). The exchange part of the Coulomb inter action, $J$ reduces the energy for two electrons with opposite spin.

Combining (a) and (b), we find that given a particular number of d-electrons as fixed by $U$ in some transition atom, the exchange $\mathrm{J}$ favors the magnetic spin aligned state; nothing but Hund's rule of atomic physics.

Even though the true atomic physics is far more complicated by effects like spin-or bit coupling etc., our interest being in impurity magnetism, we approximate the atomic Hamiltonian by,

$$
\begin{aligned}
\mathcal{H}_{\alpha}=\sum_{i \sigma} E_{\alpha} N_{i \sigma} & +U \sum_{i j} N_{i r} N_{j,-r} \\
& +\frac{1}{2}(U-J) \sum_{i \neq j} N_{i \sigma} N_{j \sigma}
\end{aligned}
$$


where $E_{d}$ is the one electron energy level and $N_{i \sigma}=a_{L r}^{+} Q_{i r}$ is the number operator for the electrons in the ith $d$ orbital. Using a single value of $E_{d}$ neglects any possible crystal field splitting. Finally, $\mathrm{H}_{\mathrm{s}-\mathrm{d}}$ characterises the metal-impurity interaction and is taken to be of the form

$$
H_{s-\alpha}=v_{\Delta-\alpha} \sum_{k i r}\left(a_{k r}^{+} a_{i r}+a_{i r}^{+} a_{k r}\right)
$$

The admixture coefficient $\mathrm{V}_{\mathrm{s}-\mathrm{d}}$ which strictly speaking is $\mathrm{k}$-dependent, represents the process in which a d-electron hops off the impurity, reducing the number of d-electrons by one, or a band electron hops on the impurity with a corresponding increase in the number of delectrons. Thus an admixture clearly tends to reduce the impurity magnetism. Physically the admixture arises from the d (or 4f) electron of the impurity experiencing the attractive potential due to the nuclei of the neighboring atoms in the metal and hence transferring over. Thus one expects $\mathrm{V}_{s-d}$ to be a sensitive function of the spatial extent of the core wave function, being much larger for 3d electrons than for $4 f$. The three terms described above thus seem to secify the physics of the problem. The next question then obviously is what is the nature of the solution? 
less understood, although only one of them has been mathematically solved. The limiting case in which the problem has been solved is when the impurity-metal interaction is the strongest so that one can treat the complicated Coulomb term in a self-consistent field approximation. Very briefly, one replaces

$$
N_{i \sigma}=\left\langle N_{i \sigma}\right\rangle+\left[N_{i \sigma}-\left\langle N_{(\sigma}\right\rangle\right]
$$

and neglects terms of the form $\left[N_{i r}-\left\langle N_{i r}\right\rangle\right] \cdot\left[N_{j \sigma}-\left\langle N_{j \sigma}\right\rangle\right]$. In this limit the d-states take the simple one electron form,

$$
E_{i \sigma}=E_{d}+(U-J) \sum_{i \neq j}\left\langle N_{j, \sigma}\right\rangle+U \sum_{j}\left\langle N_{j,-r}\right\rangle
$$

in which the averages $\left\langle N_{j} r\right\rangle$ must be determined self-consistently. Physically, such an approximation is tantamount to saying that a given electron remains in the localized state for a time sufficiently short so that it does not obtain detailed information on the occupation of the other spin state orbitals. Since the addition of an electron raises the energy by an amount of the order of the Coulomb energy $U$, the uncertainty principle requires a one-electron life time of the order of $(\hbar / U)$ in order that the self-consistent field approach be valid. Such a life time is indeed provided by the mixing interaction, which through Golden Rule yields the transition rate $W$, 
$\hbar \omega=\Delta=\pi V^{2} \rho_{\Delta}\left(E_{d}\right)$

where $\Delta$ is the width of the virtual level, $P_{s}\left(E_{d}\right)$ is the density of states of the host metal evaluated at the $d$ state energy and $v^{2}$ is the average of the square modulus of the admixture matrix element 8. It then follows that the region of validity of the Hartree-Fock self consistent field approach is limited to a range of parameters such that $U / \Delta<1$.

For smaller values of the level width the electron-electron correlations play an important role and must be taken into account. However, it is very important to realize that it is in the opposite limit i.e., $U / \Delta>1$, that a given electron remains localized on the site of the impurity atom for a relatively long time while tending to keep other electrons away via the Coulomb interaction and thereby is able to form a localized magnetic moment. The actual analysis of the mathematical problem and its detailed discussion is easily found in the literature, ${ }^{(4)}$ and any further discussion here is hardly appropriate. However, we quote here the important result that the most favorable case for magnetism occurs when the virtual level falls self-consistently at the Fermi-energy in which case the condition for forming a magnetic moment is, 


$$
\frac{1}{\pi} \cdot \frac{U+4 J}{\Delta}=1
$$

Thus, the self-consistent field approximation is valid only in the non-magnetic limit, since for larger values of $\Delta$, the impurity is non-magnetic, whereas for smaller values a magnetic moment develops.

The opposite limit $U / \Delta \gg 1$, i.e., where the local Coulombs interactions dominate is also in a qualitative sense understood, although the problem in this limit is very far from solved. The work to be described in the next few sections deals with the solution of the problem in this limit i. e., in the magnetic limit. However, instead of dealing with the actual Anderson's Hamiltonian, we shall be dealing with the so called s-d exchange model, which was originally proposed in the context of ferromagnetism of $3 \mathrm{~d}$ metals by Zener. (28) Kasuya ${ }^{(29)}$ has derived this Hamiltonian of the exchange interaction between localized and conduction electrons for dilute magnetic alloys. However, Schrieffer and Wolff $(30)$ have shown that by means of a canonical transformation it is possible to eliminate the mixing interaction to first order from the Anderson Hamiltonian, in the limit $U / \Delta>1$. The result is the $s-d$ Hamiltonian of the form 


$$
\mathcal{H}=\overline{\mathcal{H}}_{0}+\overline{\mathcal{H}}_{b-d}
$$

where

$$
\bar{H}_{0}=\sum_{k \sigma} \epsilon_{k \sigma} \bar{N}_{k \sigma}+E_{d} \sum_{\tau} \bar{N}_{d \sigma}+U \bar{N}_{d \uparrow} \bar{N}_{d \downarrow}
$$

These are simplified expressions for the case of a nondegenerate $d$-orbital. The degenerate case is considered by Coglin and Schrieffer. (31) A close look at $\overline{\mathcal{H}}_{0}$ reveals that $a_{K \sigma^{\text {states }}}$ represent a complete set of non-interacting fermions with a dispersion relation identical to that of the pure host. The se states thus form a free particle Fermi sea and as such shall contribute to the physical properties (magnetic susceptibility, specific heat etc.) by an amount numerically equal to the pure host (modified perhaps by a trivial potential scattering factor). $\overline{\mathrm{H}}_{\mathrm{s}-\mathrm{d}}$ is given by,

$$
\begin{gathered}
\bar{H}_{d-d}=\frac{1}{2 N} \sum_{k g} J_{k g}\left\{\left(\bar{a}_{k q}^{+} \bar{a}_{g q}-\bar{a}_{k \downarrow}^{+} \bar{a}_{g \downarrow}\right)\left(\bar{a}_{d q}^{+} \bar{a}_{d q}-\bar{a}_{d \downarrow}^{+} \bar{a}_{d \downarrow}\right)\right. \\
+2 \bar{a}_{k \uparrow}^{+} \bar{a}_{g \downarrow} \bar{a}_{d \downarrow}^{+} \bar{a}_{d \uparrow} \\
\left.+2 \bar{a}_{k \downarrow}^{+} \bar{a}_{g q} \bar{a}_{d q}^{+} \bar{a}_{d \downarrow}\right\}
\end{gathered}
$$


In the above,

$$
\begin{aligned}
& \bar{a}_{d \sigma}^{+}=e^{-s} a_{d \sigma}^{+} e^{s} \\
& \bar{a}_{k \sigma}^{+}=e^{-s} a_{k \sigma}^{+} e^{s}
\end{aligned}
$$

where $S$ is the generating function for the transformation. The conditions for the validity of the Schrieffer-Wolff transformation are,

$$
\frac{\left|E_{d}\right|}{\Delta} \gg 1 \quad \frac{E_{d}+U}{\Delta} \gg 1
$$

where the convention $E_{F}=0$ is being used. Thus in the limit where these conditions are fulfilled, the s-d exchange model of a well defined impurity spin interacting with the conduction electron sea is valid. One must however, be careful to note that the "Spin" is not that of the bare impurity, for the canonical transformation dresses the spin with a long conduction electron "skirt" i. e., $\bar{a}_{d} \neq a_{d r}$ The transformed operators $\bar{a}_{d \sigma}$ and $\bar{a}_{x \sigma}$ are 
quite different from the initial operator $s$ and represent states having an infinite life time and thus an infinitesimal level width as long as the above condition for validity of the transformation holds. 
III. THE s-d EXCHANGE MODEL

We have referred to the $s-d$ exchange model in the historical context of the problem of impurity magnetism in the previous section. In this section we shall present a concise, yet complete in its essential features, description of this model, and its correspondence with reality.

The s-d exchange model assumes a well defined (recall the magnetic limit of the Anderson model) localized, impurity spin $\mathrm{S}$ interacting with the conduction electron $\mathbf{s}$ pin $\mathbf{s}$ by an interaction of the form,

$$
\mathcal{H}_{s-d}=-J \Omega \vec{s} \cdot \vec{s} f(r, \theta, \phi)
$$

where $J$ is the strength of the exchange coupling, $f(r, \theta, \phi)$ represent $s$ the spatial extent of the localized moment, and $\Omega$ is the atomic volume. In the second quantized form the interaction can be written as (see Ref. 5).

$$
\begin{aligned}
H_{s-d}= & \left(\frac{-1}{N}\right) \sum_{k_{1} k_{2}} J_{k_{1} k_{2}}\left\{\left(a_{k_{1} \uparrow}^{+} a_{k_{2} \uparrow}-a_{k_{1} \downarrow}^{+} a_{k_{2} \downarrow}\right) S_{z}\right. \\
& \left.+a_{k_{1} \uparrow}^{+} a_{k_{2} \downarrow} S_{-}+a_{k_{1} \downarrow}^{+} a_{k_{2} \uparrow} S_{+}\right\}
\end{aligned}
$$

where, $a_{k \sigma}^{+}$and $a_{k \sigma}$ are the usual creation and anhilation 
operators for electrons in the state with momentum $k$ and $\mathrm{Z}$ component of the spin being $\sigma ; S_{Z}, S_{ \pm}=\left(S_{x} \pm i S_{y}\right), \quad$ are the impurity spin operator $\mathrm{s}, \mathrm{N}$ is the number of electrons, $\mathrm{J}_{\mathrm{K}_{1}} \mathrm{~K}_{2}$ is the Fourier transform of the product $J f(r, \theta, \phi)$, so that the dependence of $\mathrm{J}_{\mathrm{K}_{1} \mathrm{~K}_{2}}$ on $\mathrm{K}_{1}$ and $\mathrm{K}_{2}$ arises from the spatial dependence of $f(\gamma, \theta, \phi)$. It has been customary to assume an isotropic, zero range, $\delta$-function potential, $f(r)=\delta(r)$. In that case $J_{K_{1} K_{2}}=J$, a constant, and we have at hand the standard "contact s-d exchange interaction". However, it is most important to realize that such an as sumption restricts our consideration of phenomena well outside the impurity cell, for it is only in that region that the assumption can be expected to be justifiable. The finite $r$ ange of the $s-d$ exchange interaction can be incorporated schematically by assuming a momentum space cut-off for $\mathrm{J}_{\mathrm{K}_{1}} \mathrm{~K}_{2}$ of the form,

$$
\begin{aligned}
J_{k_{1} k_{2}} & =J:-D<\epsilon_{k_{1}}, \epsilon_{k_{2}}<D \\
& =0
\end{aligned}
$$

otherwise.

With this form of $\mathrm{J}_{\mathrm{K}_{1}} \mathrm{~K}_{2}$ we obtain the well known contact 8-d exchange interaction given by, 


$$
\begin{aligned}
H_{s-\alpha}=(-J / N) \sum_{k_{1} k_{2}} & \left\{\left(a_{k_{1} \uparrow}^{+} a_{k_{2} \uparrow}-a_{k_{1} \downarrow}^{+} a_{k_{2} \downarrow}\right) S_{z}\right. \\
+ & \left.a_{k_{1} \uparrow}^{+} a_{k_{2} \downarrow} S_{-}+a_{k_{1} \downarrow}^{+} a_{k_{2} \uparrow} S_{+}\right\}
\end{aligned}
$$

(III. 4)

which, for $J<0$ characterizes antiferromagnetic coupling and corresponds to the case of interest in the Kondo problem. The case $J>0$ is also possible and characterizes direct ferromagnetic exchange. Although, for the transition atom impurities of primary interest in dilute magnetic alloys the antiferromagnetic admixture exchange dominates, and as such has been of interest in this field, we should keep in mind the possibility of observing something inter esting for $\mathrm{J}>\mathrm{o}$ in our solutions. We shall later see that the case of ferromagnetic exchange (i.e., $J>0$ ) also has interesting formation, which has been earlier overlooked in the field simply because the approximate solutions available fail to show the se characteristics. It should be noted that the s-d exchange interaction given in (III. 4) char acterizes the interaction of conduction electrons with a single impurity atom of spin S. Thus it would be a reasonable description of the realistic system of many impurity atoms in the nonmagnetic host only if the total number of impurity atoms is very small as compared to the total number of host atoms, in which case we may safely assume that there is no "direct" interaction between the 
impurity atoms. Thus for very low impurity concentration $c$, we shall be justified in treating the quantities to be compared with experimental data as c times that obtained from the theoretical model. Experimentally the value of c differs from one system to another depending upon the particular properties of the system. It is therefore difficult to give any precise number for the upper limit on c, beyond which the "direct" impurity interactions would become important and a cimple linear extrapolation of the results obtained from this theoretical model shall no longer hold. However, from the accumulated wealth of experimental data, one might be safe in concluding that for crystalline systems $\mathrm{C}_{\max }$ is between 0.1 at. $\%$ and 0.5 at.$\%$ while for amorphous systems $\mathrm{C}_{\max }$ may go as high as 1. 5 at. \% . No theoretical attempt has been made to explain why $C_{\max }$ is higher in amorphous systems as opposed to crystalline system. Although we have not attempted to take up this que stion with any great attention in this work, we shall see in later sections that this phenomenon is readily understood as a natural consequence of the scattering approach taken here. (We shall therefore put forth, for the first time, very physical arguments to explain this phenomenon during the course of discussion of our analysis in later sections.) The interaction (III. 4) does not have a momentum conserving $\delta$-function in it. It is a consequence of the fact that in our problem one of the interacting particles is localized at the impurity site, and hence momentum is not conserved. The interaction does conserve the total spin and is rotationally invariant. 


\section{FORMULATION OF THE THEORETICAL PROBLEM}

\section{A. The Scattering Equations}

The approach we take is one of formal field theoretic scattering. Although the basic scattering equations which we are going to use are well known as the Lippmann-Schwinger equations, we shall derive them to bring out their nature in the case of field theoretic scattering explicitly. Consider the Hamiltonian $\mathrm{H}=\mathrm{H}_{\mathrm{o}}+\mathrm{V}$ where we shall refer to $\mathrm{H}_{0}$ as the unperturbed Hamiltonian and V as the interaction. In our case $\mathrm{H}_{\mathrm{o}}$ is the Hamiltonian describing the free system of $\mathrm{N}$ electrons. Let its ground state be denoted by $\mid F>$. As we have an impurity of spin $S$ in this gas of free conduction electrons, in the absence of any interaction between the conduction electrons and the impurity, we can specify the impurity atom state by $\left|S_{Z}\right\rangle$, the $Z$-component of its spin. We shall therefore denote the ground manifold of the non-interacting conduction electron and impurity system by $\left(\mathbf{F} \mathrm{S}_{\mathrm{Z}}\right)$. This ground state is obviously $(2 \mathrm{~S}+1)$ fold degenerate.

Consider now an additional electron with energy $\epsilon_{K}$ above the Fermi level incident on the target, which is in one of the $(2 S+1)$ ground states, $\quad \mid F S_{Z}>$. With our notation, we may write the incident state as,

$$
a_{k r}^{+}\left|F_{i} S_{k}\right\rangle
$$


with

$$
\mathcal{H}_{0} a_{k \sigma}^{+}\left|F_{j} s_{z}\right\rangle=\left(\varepsilon_{0}+\epsilon_{k}\right) a_{k \sigma}^{+}\left|F_{j} s_{z}\right\rangle
$$

where $\varepsilon_{0}$ is the ground state energy of the non-interacting sy stem of $\mathrm{N}$ electrons.

We now wish to construct exact outgoing and incoming scattering states. Let us denote such a state by $\left|\omega, k^{\prime} r^{\prime}, S_{z}^{\prime}\right\rangle$ with

$$
\mathcal{H}\left|\omega, k^{\prime} \sigma^{\prime}, s_{z}^{\prime}\right\rangle=E_{k^{\prime}}\left|\omega, k^{\prime} \sigma_{;}^{\prime} s_{z}^{\prime}\right\rangle
$$

Here, $\omega$ characterizes the state of the $\mathrm{N}$ electron system after the incident electron $K \sigma$ has scatter ed to the state $K^{\prime} r^{\prime}$, and $S_{Z}^{\prime}$ the state of the impurity. In the usual way, we write the exact outgoing and incoming states as

$$
\left|\omega, k^{\prime} \sigma^{\prime} ; s_{z}^{\prime}\right\rangle^{ \pm}=a_{k \sigma}^{+}\left|F_{j} s_{z}\right\rangle+|x\rangle^{ \pm}
$$

where + and - refer to outgoing and incoming states respectively. From (2) and (3), we then have,

$$
\mathcal{H}\left\{a_{k r}^{+}\left|F_{j} s_{k}\right\rangle+|x\rangle^{ \pm}\right\}=E_{k}\left\{a_{k r}^{+}\left|F_{j} s_{z}\right\rangle+|x\rangle^{ \pm}\right\}
$$


or

$$
\begin{aligned}
& \left\{H_{0}+V-E_{k}\right\} \cdot\left\{a_{k \sigma}^{+}\left|F_{j} S_{z}\right\rangle\right\}=\left(E_{k}-H\right)(x\rangle^{ \pm} \\
& \left\{E_{0}+\epsilon_{k}-E_{k}+V\right\} \cdot\left\{a_{k \sigma}^{+}\left|F_{j} S_{z}\right\rangle\right\}=\left(E_{k}-H\right)(x\rangle^{ \pm}
\end{aligned}
$$

or

$$
\therefore|x\rangle^{ \pm}=\frac{1}{E_{k}-\mathcal{H} \pm i \eta}\left(\varepsilon_{0}+\epsilon_{x}-E_{k}+v\right) \cdot\left\{a_{k \sigma}^{+}\left|F_{j} s_{z}\right\rangle\right\}
$$

Thus, we have from (3)

$$
\begin{aligned}
\left|\omega, k r_{j} s_{z}\right\rangle^{ \pm}=a_{k r}^{+}\left|F_{j} s_{z}\right\rangle & +\frac{1}{E_{k}-\mathcal{H} \pm i \eta} \\
& \left(\varepsilon_{0}+\epsilon_{k}-E_{k}+V\right) \cdot\left\{a_{k r}^{+}\left|F_{j} s_{z}\right\rangle\right\}
\end{aligned}
$$

These are the basic scattering equations. However, it is to be noted that in the event that the energy spectrum of Ho and $H$ happen to coincide, then and only then shall we have $\left(\varepsilon_{0}+\epsilon_{x}\right)=E_{k}$, in which case (4) shali reduce to,

$$
\left|\omega, k \sigma ; s_{z}\right\rangle^{ \pm}=a_{k \sigma}^{+}\left|F_{j} s_{z}\right\rangle+\frac{1}{E_{x}-H \pm i \eta} \vee\left\{a_{k r}^{+}\left|F_{j} s_{z}\right\rangle\right\}
$$

the usual form of Lippman-Schwinger scattering equations to be found in the texts. 
It is therefore important to realize that in the case of field theoretic scattering, when the energy corrections (selfenergy in the conventional terminology) need not be infinitesimal, (4) gives the correct form of the scattering equations. Physically, the content of this statement is the following; In the case of field theoretic scattering, the interaction extends to infinity, and hence the energy of the colliding systems, even at infinite separation is

not given by, e.g. $\left(\varepsilon_{0}+\epsilon_{K}\right)$ in our case, but by $E_{K}$. Thus in order to describe the scattering process correctly, one has to assign the correct energy to the incident train, by formally modifying Ho so as to coincide its energy spectrum with that of $\left(\mathrm{H}_{\mathrm{o}}+\mathrm{V}\right)$.

To bring this out more clearly, we put equation (4) in a different form. After a few simple manipulations we may write equation (4) in the form

$$
\begin{aligned}
\left|\omega, k \sigma, s_{z}\right\rangle^{ \pm}=\frac{i \eta}{E_{k}-H_{0} \pm i \eta} a_{k \sigma}^{+}\left|F_{j} s_{z}\right\rangle & \\
& +\frac{1}{E_{k}-H_{0} \pm i \eta} V\left|\omega, k r_{j} s_{z}\right\rangle^{ \pm}
\end{aligned}
$$

This is then the final form of the scattering equations we shall be using. On operating $\left(F_{k}-H_{0} \pm i \eta\right)$ on both sides of (IV.5) one immediately sees that $\left|\omega, k \sigma_{j} s_{\xi}\right\rangle^{ \pm}$are indeed eigen functions of $\mathrm{H}$. 
B. Notation For Many Electrons + Impurity Wave Functions

As usual we shall write the $\mathrm{N}$-electron wave function of the unperturbed Hamiltonian $\mathrm{H}_{0}$, as an antisymmetric product of $\mathrm{N}$ single electron wave functions. At any given temperature $T$, we shall take the occupied single electron states to be determined by the most probable distribution, the Fermi distribution at that temperature. Thus the $\mathrm{N}$-electron wave function which we shall denote by $\phi_{N}$ for brevity of notation, is to be taken as,

$$
\left|\phi_{N}\right\rangle=A \prod_{i=1}^{N}\left|\Phi_{k_{i} \sigma_{i}}\right\rangle
$$

where $A$ is the usual antisymmetrization operator and $\left|\varphi_{k_{i}} \sigma_{i}\right\rangle$ denote the single electron states. In the second quantized notation we may then write,

$$
\begin{aligned}
\left|\phi_{N}\right\rangle & =a_{k_{1} \sigma_{1}}^{+} a_{k_{2} \sigma_{2}}^{+} \cdots \cdot a_{k_{N} \sigma_{N}}^{+}|0\rangle \\
& \equiv\left|k_{1} \sigma_{1}, k_{2} \sigma_{2}, \cdots \cdot k_{N} \sigma_{N}\right\rangle
\end{aligned}
$$

where $|0\rangle$ is the vacuum state assumed to be normalized to unity. ${ }^{(33)}$ Also $a_{k r}|0\rangle \equiv 0$, and the a and $a^{+}$obey the usual anticommuntation rules. Thus, the unperturbed state of the system of $\mathrm{N}$-electron gas, an impurity atom in the state $\left|\mathrm{S}_{Z}\right\rangle$, with an 
additional electron incident on this system in the single particle state $|k r\rangle$, shall be denoted by

$$
\left|\phi_{N}, k \sigma_{j}, s_{Z}\right\rangle
$$

During the scattering process, the state of the $\mathrm{N}$-electron system can change due to the excitation of particle-hole pairs. Such changes in $\phi_{N}$ shall be denoted by

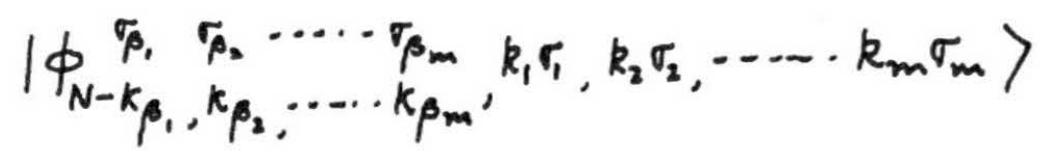

where the Greek suffixes, $\beta$, to $\beta_{m}$, on $k$ and $\sigma$ denote those states from which electrons are knocked out of their original configuration $\phi_{N^{\prime}}$ and $k_{1} \sigma_{1}$ to $k_{m} \sigma_{m}$ denote the states which are now being occupied by these m-electrons. Thus the occurrence of any Greek suffix indicates the absence of an electron or what is equivalent, the presence of a hole. Thus (7) denotes the mth excited state of the unperturbed Hamiltonian Ho and represents a state with $\mathrm{m}$ (particle + hole) pairs.

In the present problem we are interested only in considering the scattering of a particie from an initial single particle state to a final single particle state, since this scattering amplitude is sufficient to calculate the physical properties of the system. In the language of Green's functions this is equivalent to considering only 
single particle propagators. (27) But this does not mean that the $8-d$ exchange scattering can be treated within one-electron theory, for an exact knowledge of even the single particle to single particle scattering amplitudes requires a knowledge of all higher order scattering processes, a feature typical of any many body phenomenon. The exact scattering state corresponding to the initial state we shall denote by $\left|\psi_{k}\right\rangle$ where $\left|\psi_{k}\right\rangle$ now describes the interacting system of $\mathrm{N}$-electron gas, the impurity and the additional electron $|k \sigma\rangle$.

With this notation, the scattering equation for our problem reads,

$$
\begin{aligned}
\left|\Psi_{k}\right\rangle^{ \pm}=\frac{i \eta}{E_{k}-\mathcal{H}_{0} \pm i \eta} & \cdot\left|\phi_{N_{1}} k \sigma ; S_{z}\right\rangle \\
& +\frac{1}{E_{K}-\mathcal{H}_{0} \pm i \eta} \mathcal{H}_{\Delta-\alpha}\left|\psi_{k}\right\rangle^{t}
\end{aligned}
$$

and shall be the starting point of the solution of the problem in the next section.

C. The Exact Single Particle Scattering Equations

Our scattering equation, still in the operator form, reads

$$
\begin{aligned}
\left|\psi_{k}\right\rangle=\frac{i \eta}{E_{\alpha}-\mathcal{H}_{0}+i \eta} \mid & \left|\phi_{N}, k i, s_{z}\right\rangle \\
& +\frac{1}{E_{K}-\mathcal{H}_{0}+i \eta} H_{s-\alpha}\left|\psi_{K}\right\rangle
\end{aligned}
$$


From now on we shall drop the $(t)$ on $\left|\psi_{k}\right\rangle$.

Consider the electron $\mathrm{kr}$ to be incident in the state $\mathrm{K} \uparrow$ the N-electron system being described by $\phi_{N}$ and the impurity by $\mathrm{S}_{\mathrm{Z}}$. After the scattering process the electron $\mathrm{K} \uparrow$ can go into the state $\mathrm{K}^{\prime} \uparrow \quad$ or $\mathrm{K}^{\prime} \downarrow$, the former corresponding to spin nonflip scattering and the latter to spin-flip. Also appropriate modifications in the final state of the $\mathrm{N}$-electron system and impurity state would also result. Our aim is to determine the scattering amplitudes for the final states $\left|\phi_{N^{\prime}}, K^{\prime} \uparrow ; S_{Z}\right\rangle$, and $\left|\phi_{N^{\prime}} K^{\prime} \downarrow ;\left(S_{Z^{+1}}\right\rangle\right\rangle$. For spin flip scattering, conservation of the $\mathrm{Z}$-component of total spin requires $S_{Z}$ to change to $\left(S_{Z}+1\right)$. The occurrence of $\phi_{N}$ in final states denotes the fact that we are interested in the scattering process where the background medium of $\mathrm{N}$-electrons returns to its initial configuration after the scattering process is over. Let us now ask for the projection of the exact state $\left|\psi_{k}\right\rangle$ on the two final states of interest. We then obtain from(IV. 9) the following two scattering equations describing the above mentioned process;

$$
\begin{aligned}
\left\langle\phi_{N_{1}} k^{\prime} \uparrow_{j}, s_{z} \mid \psi_{k}\right\rangle= & \frac{i \eta}{E_{k}-\left(\varepsilon_{0}+\epsilon_{k^{\prime}}\right)+i \eta}\left\langle\phi_{N_{1}} k^{\prime} \uparrow, s_{z} \mid \phi_{N}, k \uparrow, s_{z}\right\rangle \\
& +\frac{1}{E_{k}-\left(\xi_{0}+\epsilon_{k^{\prime}}\right)+i \eta}\left\langle\phi_{N_{1}} k^{\prime} \varphi_{j} s_{z}\left|\mathcal{H}_{\Delta-d}\right| \psi_{k}\right\rangle
\end{aligned}
$$




$$
\begin{aligned}
\left\langle\phi_{N_{0}} k^{\prime} \downarrow,\left(S_{z}+1\right) \mid \psi_{k}\right\rangle & =\frac{i \eta}{E_{k}-\left(\varepsilon_{0}+\epsilon_{k}\right)+i \eta}\left\langle\phi_{N_{1}} k^{\prime} \downarrow,\left(S_{z}+1\right) \mid \phi_{N}, k \eta, S_{z}\right\rangle \\
& +\frac{1}{E_{k}-\left(\varepsilon_{0}+\epsilon_{k}\right)+i \eta}\left\langle\phi_{N}, k^{\prime} \downarrow ;\left(S_{z}+1\right)\left|H_{\Delta-d}\right| \psi_{k}\right)
\end{aligned}
$$

where we have used the fact that $\left|\phi_{\mathrm{N}}, \mathrm{K}^{\prime} \uparrow ; \mathrm{S}_{\mathrm{Z}}\right\rangle$ and $\mid \phi_{N^{\prime}} K^{\prime} \downarrow ;\left(S_{Z}+1\right)>\quad$ are eigen functions of Ho with energy $\left(\varepsilon_{0}+\epsilon_{x^{\prime}}\right)$. Further using the orthnormality of the eigen function of $\mathrm{H}_{\mathrm{O}}$, we have

$$
\begin{aligned}
\left\langle\phi_{N_{1}} k^{\prime} \uparrow_{i} s_{z} \mid \psi_{k}\right\rangle=\frac{i \eta \delta_{k K^{\prime}}}{E_{k}-\left(\varepsilon_{0}+\epsilon_{\alpha^{\prime}}\right)+i \eta}+ & \left\{\frac{1}{E_{k}-\left(\varepsilon_{0}+\epsilon_{k^{\prime}}\right)+i \eta}\right\} \\
& \left\langle\phi_{N_{1}} k^{\prime} \varphi_{j} s_{z}\left|H_{\Delta-\alpha}\right| \psi_{k}\right\rangle
\end{aligned}
$$

and

$$
\begin{aligned}
& \left\langle\phi_{N_{1}} k^{\prime} \downarrow ;\left(s_{z}+1\right) \mid \psi_{k}\right\rangle=\left\{\frac{1}{E_{k}-\left(\varepsilon_{0}+\epsilon_{k^{\prime}}\right)+i \eta}\right\} \\
& \cdot\left\langle\phi_{N_{1}} k^{\prime} \downarrow,\left(s_{z}+1\right)\left|H_{\Delta-\alpha}\right| \psi_{k}\right\rangle
\end{aligned}
$$

We call the quantities $\left\langle\phi_{N}, R^{\prime} \uparrow, S_{Z}\left|\mathcal{H}_{\Delta-\alpha}\right| \psi_{k}\right\rangle$ and $<\phi_{N}, k^{\prime} \downarrow,\left(s_{q}+1\right)\left|\mathcal{K}_{\Delta-\alpha}\right| \psi_{K}>$ the spin non-flip and spin-flip scattering amplitudes and shall denote them by $t_{\text {uf }}$ and $t_{f}$ respectively. The aim of the problem is to obtain solutions for these two scattering amplitudes. To this effect we introduce a complete set of eigen functions of the unperturbed hamiltonian $H_{0}$ between $\mathrm{H}_{\mathrm{s}-\mathrm{d}}$ and $\left|\psi_{\mathrm{K}}\right\rangle$ 
to obtain,

$$
\begin{gathered}
t_{n f}=\sum_{n} \sum_{k^{\prime \prime} r^{\prime \prime} s_{z}^{\prime \prime}}\left\langle\phi_{N}, k^{\prime} \varphi_{j} s_{z}\left|\mathcal{H}_{\beta-d}\right| n(p, h), k^{\prime \prime} \sigma^{\prime \prime}, s_{z}^{\prime \prime}\right\rangle \\
\left\langle n(p, h), k^{\prime \prime} \sigma^{\prime \prime} ; s_{z}^{\prime \prime} \mid \psi_{k}\right\rangle
\end{gathered}
$$

and

$$
\begin{array}{r}
t_{f}=\sum_{n} \sum_{k^{\prime \prime} \sigma^{\prime \prime} s_{z}{ }^{\prime \prime}}\left\langle\phi_{N_{1}} k^{\prime} \downarrow,\left(s_{z}+1\right)\left|H_{s-\alpha}\right| n(\rho, h), k^{\prime \prime} \sigma^{\prime \prime} s_{z}^{\prime \prime}\right\rangle \\
\cdot\left\langle n(\rho, h), k^{\prime \prime} \sigma_{j}^{\prime \prime}, s_{z}^{\prime \prime} \mid \psi_{k}\right\rangle
\end{array}
$$

where $\left.\quad K^{\prime \prime} \sigma^{\prime \prime} ; \mathrm{n}(\mathrm{p}, \mathrm{h}) ; \mathrm{S}_{\mathrm{z}}{ }^{\prime \prime}\right\rangle \quad$ refers to an eigen function of $\mathrm{H}_{\mathrm{O}}$ with one electron in the state $k$ " $\sigma$ " and $\mathrm{n}$ (particle + hole) pairs excited, with the impurity in the state $S_{Z}$ ". The sum over $n$ refers to a sum over all possible pair excitations. As an illustration (IV.7) gives explicitly the eigen function for a particle $+m$ (particle + hole) pairs and the sum over $\mathrm{n}$ for this particular eigen function means a sum over all $\mathrm{m} \mathrm{k}^{\prime} \mathrm{s}$ and $\sigma^{\prime} \mathrm{s}$.

At this point it is very important to note a simplifying feature of our interaction Hamiltonian $\mathrm{H}_{\mathrm{s}-\mathrm{d}}$, which restricts the set of intermediate states in (IV.14) and (IV.15) to single particle and single particle + one (particle + hole) pair states. From the form of $\mathrm{H}_{\mathrm{s}-\mathrm{d}}$, equation (III. 4), we note that it is bilinear in the electron creation and anhilation ope rator. As such, when sandwiched between any two eigen states of $\mathrm{H}_{0}$, it can give a non-vanishing contribution only if the states on either side differ by at most one electron + one (electron + hole) pair. If the states differ by more than 
one electron + one (electron + hole) pair, the matrix element shall vanish because of the orthogonality of the eigen functions of $\mathrm{H}_{\mathrm{O}}$. Thus in the case of equations (IV. 14) and (IV. 15), where we have a single particle state on the left, the only intermediate states that can give a non-vanishing contribution are one particle, and one particle + one (particle + hole) pair states.

To show this behaviour explicitly let us consider the following matrix element,

$$
\left\langle\phi_{N_{1}} k^{\prime} q_{j} s_{z}\left|\mathcal{H}_{\Delta-d}\right| \phi_{N}-k_{\beta_{1}}^{r_{\beta_{1}}},{ }_{k_{\beta_{2}}}^{\sigma_{\beta_{2}}}, k_{i} \sigma_{i}, k_{j} \sigma_{j}, k^{\prime \prime} \sigma^{\prime \prime} ; s_{z}^{\prime \prime}\right\rangle
$$

which would occur in (IV. 14) as the contribution of a one electron +2 (electron + hole) pair state. Let us now introduce the explicit form of $\mathrm{H}_{\mathrm{s}-\mathrm{d}}$, and for purposes of illustration consider one of the terms, $a_{k, \uparrow}^{+}$ $a_{\mathrm{k}_{2} \text { sof }} \mathrm{H}_{\mathrm{s}-\mathrm{d}^{\circ}}$. Then we have the following matrix element at hand.

$$
\left\langle\phi_{N_{1}} k^{\prime} \uparrow, S_{z}\left|\sum_{k_{1} K_{2}} a_{k_{1} \varphi}^{+} a_{\left.k_{2}\right\}} s_{z}\right| \phi_{N-k_{\beta_{1}}, k_{\beta_{2}}}^{r_{\beta_{1}}}, r_{\beta_{2}} k_{i} \sigma_{i}, k_{j} \sigma_{j}, k^{\prime \prime} \sigma^{\prime \prime} ; s_{z}^{\prime \prime}\right\rangle
$$

Mere observation of this matrix elements shows that the interaction term can at most convert the R. H. S. wave function to a single electron + one (electron + hole) pair state, thus giving the following inner product, 
$-36-$

$$
\left\langle\phi_{N}, k^{\prime} \uparrow, s_{z} \mid \phi_{N-k_{\beta_{1}}}^{\sigma_{\beta_{1}}}, k_{i} \sigma_{i}, k^{\prime} \uparrow, s_{z}\right\rangle
$$

which is identically zero because of the orthogonality of eigen functions of $\mathrm{H}_{\mathrm{O}}$. It is therefore clear that the contribution of all electron $+n$ (electron + hole) pair states for $n \geqslant 2$ is identically zero in equation (IV. 14) and (IV. 15).

Introducing single electron and single electron + one (electron + hole) pair states explicitly in (IV. 14) and (IV. 15), we have, $t_{n f}=\left[\sum_{k^{\prime \prime} r^{\prime \prime} s_{z}^{\prime \prime}}\left\langle\phi_{N}, k^{\prime} \uparrow, s_{z}\left|H_{s-d}\right| \phi_{N}, k^{\prime \prime} \sigma_{j}^{\prime \prime} s_{z}^{\prime \prime}\right\rangle\right.$
$\cdot\left\langle\phi_{N}, k^{\prime \prime} r^{\prime \prime}, s_{z}^{\prime \prime} \mid \psi_{k}\right\rangle$

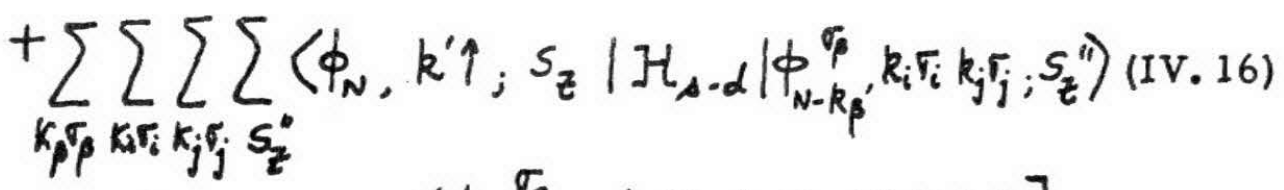

$$
\left.\cdot\left\langle\phi_{N-k_{\beta}}^{\sigma_{p}}, k_{i} \sigma_{i}, k_{j} \sigma_{j}, S_{z}^{\prime \prime} \mid \psi_{k}\right\rangle\right]
$$

and,

$$
\begin{aligned}
& t_{f}=\left[\sum_{k^{\prime \prime} \sigma^{\prime \prime} s_{z}^{\prime \prime}}\left\langle\phi_{N}, k^{\prime} l ; s_{z}+1\right)\left|H_{\Delta-d}\right| \phi_{N}, k^{\prime \prime} \sigma^{\prime \prime} ; s_{z}^{\prime \prime}\right\rangle \\
& \left\langle\phi_{N}, k^{\prime \prime} \sigma^{\prime \prime} ; S_{z}^{\prime \prime} \mid \psi_{k}\right\rangle \\
& +\sum \sum \sum \sum\left\langle\phi_{N} k^{\prime} \psi_{j}\left(\delta_{z}+1\right)\left|H_{B-\alpha}\right| \phi_{N-k_{\beta}}^{\sigma_{\beta}}, k_{i} \sigma_{i}, k_{j} \sigma_{j} ; S_{z}^{\prime \prime}\right\rangle \\
& k_{\beta} \sigma_{\beta} k_{i} r_{i} k_{j} \sigma_{j} s_{z}^{\prime \prime} \\
& \left.\cdot\left\langle\phi_{N} \cdot k_{\beta}, k_{i} F_{i}, k_{j} \sigma_{j} ; s_{z}^{\prime \prime} \mid \psi_{k}\right\rangle\right]
\end{aligned}
$$


In these expressions, it is to be noted that neither $k_{i} \sigma_{i}$ nor $k_{j} \sigma_{j}$ can be equal to $k_{\beta} \sigma_{\beta}$ in the contribution of electron + (electron + hole) pair states, for in that case they shall reduce the one electron states. Further, $k_{i} \neq k_{j}$ for $\sigma_{i}=\sigma_{j}$, for in that case the wave function is identically zero because of its antisymmetry property.

We are now in a position to evaluate the expressions (IV. 16) and (IV. 17) by explicitly introducing $\mathrm{H}_{\mathrm{s}-\mathrm{d}^{*}}$ Let us first consider the contribution of single electron states in (IV.16). We have then,

$$
\begin{array}{r}
t_{n f}^{(1)}\left(k k^{\prime}\right)=\sum_{k^{\prime \prime} \sigma^{\prime \prime} s_{z}^{\prime \prime}}\left\langle\phi_{N}, k^{\prime} \varphi_{j} s_{z}\right)\left(-\frac{J}{N}\right) \sum_{k_{1} k_{2}}\left\{\left(a_{k_{1} \uparrow}^{+} a_{k_{2} \uparrow}-a_{k_{1},}^{+} a_{k_{2},}\right) s_{z}\right. \\
\left.+a_{k_{1} \uparrow}^{+} a_{k_{2} \downarrow} s_{-}+a_{k_{1} \downarrow}^{+} a_{k_{2} \uparrow} s_{+}\right\}\left|\phi_{N} k^{\prime \prime} \sigma_{j}^{\prime \prime} s_{z}^{\prime \prime}\right\rangle \\
\cdot\left\langle\phi_{N_{1}} k^{\prime \prime} \sigma^{\prime \prime}, s_{z}^{\prime \prime} \mid \psi_{k}\right\rangle
\end{array}
$$

where the suffix (1) on $t_{n f}^{(1)}\left(K K^{\prime}\right)$ denotes the fact that we are presently considering the contribution of single electron states only. Looking at the matrix element,

$$
\left\langle\phi_{N}, k^{\prime} \uparrow, s_{z}\left|\mathcal{H}_{\Delta-d}\right| \phi_{N}, k^{\prime \prime} \sigma^{\prime \prime}, s_{z}^{\prime \prime}\right\rangle
$$

one notices that since the final state is spin non flip, namely $\left|\phi_{N}, k^{\prime} \uparrow ; S_{z}\right\rangle$ the only terms in the interaction that can give a non-vanishing contribution are those that change the intermediate state 
$\left|\phi_{N^{\prime}} k^{\prime \prime} \sigma^{\prime \prime}, S_{Z} "\right\rangle$ to the final state $\left|\phi_{N^{\prime}} k^{\prime} \uparrow ; S_{Z}\right\rangle$. We have two such terms, $\left(a_{\mathrm{K}_{1} \uparrow}^{+} a_{\mathrm{K}_{2 \uparrow}}-a_{\mathrm{K}_{1} \downarrow}^{+} a_{\mathrm{K}_{2}{ }^{\dagger}}\right) \mathrm{s}_{\mathrm{Z}}$ and $a_{\mathrm{K}_{1} \uparrow}^{+} a_{\mathrm{K}_{2} \downarrow} \mathrm{s}_{-} \quad$ for which the intermediatr states $\mid \phi_{\mathrm{N}} \mathrm{k}^{\prime \prime \uparrow ; \mathrm{S}_{\mathrm{Z}}}>$ and $\left|\phi_{N} k^{\prime \prime} \downarrow ;\left(S_{Z}+1\right)\right\rangle$ shall give non-vanishing contribution. Thus we have,

$$
\begin{aligned}
& t_{u_{f}}^{(i)}\left(k, x^{\prime}\right)=\left[\sum _ { k ^ { \prime \prime } } \left(\phi_{N_{1}} k^{\prime} \uparrow, s_{z} \mid\left(\frac{-J}{\pi}\right)\left\{\sum_{k_{1}, k_{2}}\left(a_{k_{1} \uparrow}^{+} a_{k_{2} \uparrow}-a_{k_{1},}^{+} a_{k_{2},}\right) s_{z}\right\}\right.\right. \\
& \left|\phi_{N}, k^{\prime \prime} \varphi_{j} s_{Z}\right\rangle\left\langle\phi_{N}, k^{n} \uparrow_{j} s_{Z} \mid \psi_{k}\right\rangle \\
& +\sum_{\kappa^{\prime \prime}}\left\langle\phi_{N_{1}} k^{\prime} \eta_{j} s_{z}\left|\left(-\frac{J}{N}\right)\left\{\sum_{k_{1}, k_{2}} a_{k_{1} \uparrow}^{+} a_{k_{2} \downarrow} s_{-}\right\}\right| \phi_{N_{1}} k^{\prime \prime} \downarrow ;\left(S_{z}+1\right)\right\rangle \\
& \left.\cdot\left\langle\phi_{N}, k^{\prime \prime} \downarrow ;\left(S_{z}+1\right) \mid \psi_{k}\right\rangle\right]
\end{aligned}
$$

Noting that the only non-vanishing contribution from $a_{k, 6}^{+} a_{k_{2}} b_{z}$ can come when this term destroys and creates, in the same states, one of the particles occupying one of the states in $\phi_{N}$ we may consider the action of the term $\sum_{k_{1} k_{2}}\left(a_{k_{1} \uparrow}^{+} a_{k_{2} \uparrow}-a_{k_{1} \downarrow}^{+} a_{k_{2} \downarrow}\right) s_{z}$, which we shall henceforth refer to as the static part of the interaction, in two parts. First when $\mathrm{K}_{1}$ and $\mathrm{K}_{2}$ both act on $\phi_{\mathbb{N}}$ and second when $\mathrm{K}_{1}$ and $\mathrm{K}_{2}$ both act on $k^{\prime \prime}$. The other possibility namely one of $k_{1}, k_{2}$ acting on $\phi_{N}$ and other creating a new particle in a state different from those already in $\phi_{N}$, shall give a zero contribution because of the orthogonality of the single particle and other 
excited states.

With these realizations, working out the matrix elements in the usual way, we obtain

$$
\begin{aligned}
= & {\left[( - J / N ) M \left\{\sum_{k^{\prime \prime}}\left\langle\phi_{N}, k^{\prime \prime}\right\rangle_{j} s_{z}\left|\psi_{k}\right\rangle\right.\right.} \\
& \left.\left.+\left(\sum_{k_{\beta}} n_{k_{\beta} \uparrow}-\sum_{k_{\beta}} n_{k_{\beta} \downarrow}\right) \cdot\left\langle\phi_{N}, k^{\prime} \uparrow, S_{z} \mid \psi_{k}\right\rangle\right\}\right]
\end{aligned}
$$

Where $M$ is the eigen value of $S_{Z}$ and $\sum_{k_{\beta}} n_{k_{\beta}}, \sum_{k_{\beta}} n_{k_{\beta}}$ respectively denote the number of total spin-up and spin-down electrons.

The second term, $a_{k, \uparrow}^{+} a_{k_{2} \downarrow} s_{-}$cannot give any non-vanishing contribution for $\kappa_{1}$ and $k_{2}$ acting on $\phi_{N}$ because of reasons of orthogonality of wave functions. This term contributes when $k_{2}$ becomes $\mathbf{k}^{\prime \prime}$ and $\mathbf{k}_{1}$ then creates in the state $K^{\prime}$ giving

$$
\begin{aligned}
=(-J / N) & \sqrt{(S-M)(S+M+1)} \\
& \cdot \sum_{k=1}\left\langle\phi_{N}, k^{\prime \prime} \downarrow ;\left(S_{z}+1\right) \mid \psi_{k}\right\rangle
\end{aligned}
$$

where $\sqrt{(S-M)(S+M+1)}$ is the eigen value of $S$ - acting over $\mid\left(S_{Z}+1\right)>$ which changes the $Z$ - component of impurity spin to $S_{Z}$, the final 
state Z-component.

Thus we have the spin non-flip $t$ matrix in single particle intermediate states as,

$$
\begin{aligned}
t_{n_{f}}^{(1)}\left(k, k^{\prime}\right)=(-J / N)\left[M \sum_{k^{\prime \prime}}\left\langle\phi_{N}, k^{\prime \prime} \uparrow, s_{z} \mid \psi_{k}\right\rangle\right. \\
+\sqrt{(s-M)(s+M+1)} \sum_{k^{\prime \prime}}\left\langle\phi_{N_{1}} k^{\prime \prime} \downarrow ;\left(s_{z}+1\right) \mid \psi_{k}\right\rangle \\
\left.+M\left(n_{\uparrow}-n_{f}\right) \cdot\left\langle\phi_{N^{\prime}}, k^{\prime} \uparrow ; s_{z} \mid \psi_{k}\right\rangle\right]
\end{aligned}
$$

where

$$
\begin{aligned}
& n_{\uparrow}=\sum_{k_{\beta}} n_{k_{\beta} \uparrow} \\
& n_{\downarrow}=\sum_{k_{\beta}} n_{k_{\rho} \downarrow}
\end{aligned}
$$

Next, we shall evaluate the contributions of the only other intermediate states in equation (IV.16), namely the single particle + one (electron + hole) pair states. We shall refer to these states as three particle states, treating electrons and holds on the same footing. The 3 particle contribution to $t_{n f}\left(k, k^{\prime}\right)$ is the second term of (IV.16).

There are four kinds of possible 3 particle intermediate state 8 , consistent with the conservation requirement on the $Z$ components of the spins. These are, 


$$
\begin{aligned}
& \left|\phi_{N-k_{\beta}}^{(\uparrow)}, k_{i} \uparrow, k_{j} \uparrow, s_{z}\right\rangle \\
& \left|\phi_{N-k_{\beta}}^{(\uparrow)}, k_{i} \uparrow, k_{j} \downarrow ;\left(S_{z}+1\right)\right\rangle \\
& \left|\phi_{N-k_{\beta}}^{(\omega)}, k_{i} \downarrow ; k_{j} \uparrow, S_{z}\right\rangle \\
& \left|\phi_{N-k_{\beta}}^{(t)}, k_{i} \uparrow, k_{j} \uparrow ;\left(S_{z}-1\right)\right\rangle
\end{aligned}
$$

We note again that in these intermediate states $\mathrm{Ki} \neq \mathrm{kj}$ for the first and fourth kind where $\sigma_{i}=\sigma_{j}$ because of the antisymmetry of the wave functions.

A non vanishing contribution from these intermediate states can arise only if the 3 particle state is changed to the final one particle state $\mid \phi_{\mathrm{N}_{1}} \mathrm{k}^{\prime} \uparrow ; \mathrm{S}_{\mathrm{Z}}>$ by the action of the interaction $\mathrm{H}_{\mathbf{s}-\mathrm{d}}$. In other words, $\mathrm{H}_{\mathrm{s}-\mathrm{d}}$ must destroy the (electron + hole) pair state in the intermediate 3 particle states. As an illustration, let us consider the intermediate state $\left|\phi_{\mathrm{N}-\mathrm{k}_{\beta}}^{(\uparrow)} \mathrm{k}_{\mathrm{i}} \uparrow, \mathrm{k}_{\mathrm{j}} \uparrow ; \mathrm{S}_{\mathrm{Z}}\right\rangle$. This gives the contribution to $\mathrm{t}_{\mathrm{nf}}^{(3)}\left(\mathrm{K}, \mathrm{K}^{\prime}\right)$ as,

$$
\begin{aligned}
\sum_{k_{\beta}} \sum_{k_{i}} \sum_{k_{j}}\left\langle\phi_{N}, k^{\prime} \uparrow_{j} s_{z}\left|H_{\mu-d}\right| \phi_{N-k_{\beta}}^{(\uparrow)}, k_{i} \uparrow, k_{j} \uparrow ; s_{z}\right\rangle \\
\cdot\left\langle\phi_{N-k_{\beta}}^{(\uparrow)}, k_{i} \uparrow, k_{j} \uparrow ; s_{z} \mid \psi_{k}\right\rangle
\end{aligned}
$$

Since no spin changes occur for these states, the only contribution comes from the term $\sum_{k_{1} k_{2}} a_{k_{1} \uparrow}^{+} a_{k_{2} \uparrow} S_{z}$ of $\mathrm{H}_{8-\mathrm{d}}$ and is given by, 


$$
\begin{aligned}
(-J / N) M & {\left[\sum_{k_{\beta}} \sum_{k_{i}}\left\langle\phi_{N-k_{\beta}}^{(\uparrow)}, k_{i} \uparrow, k^{\prime} \uparrow, s_{z} \mid \psi_{k}\right\rangle\right.} \\
& \left.+\sum_{k_{\beta}} \sum_{k_{j}}\left\langle\phi_{N-k_{\beta}}^{(\uparrow)}, k_{j} \uparrow, k^{\prime} \uparrow, s_{z} \mid \psi_{k}\right\rangle\right]
\end{aligned}
$$

We have two terms arising from the fact that there are direct and exchange terms for the two electrons $k_{i} \uparrow, k_{j} \uparrow$. Further, since

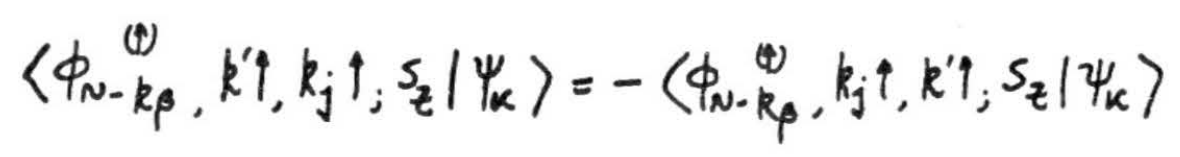

because of antisymmetry, we may write the contribution of the intermediate states $\left|\phi_{N-k_{p}}^{(p)}, R_{i} \boldsymbol{T}_{,} k_{j} \phi_{j} s_{z}\right\rangle \quad$ as,

$$
2(-J / N) M \sum_{k_{\beta}} \sum_{k_{i}}\left\langle\phi_{N}^{(\uparrow)} k_{\beta}, k_{i} \uparrow, k^{\prime} \eta_{j} S_{z} \mid \psi_{k}\right\rangle
$$

Similar considerations give the contributions of the 2 nd, $3 r d$ and 4 th type of three particle states as (in the same order)

$$
2(-J / N) \sqrt{(S-M)(S+M+1)} \cdot \sum_{k_{\beta} k_{i}}\left\langle\phi_{N-k_{\beta}}^{(\tau)} k_{i} \downarrow, k^{\prime} \eta_{j}\left(S_{z}+1\right) \mid \psi_{u}\right\rangle
$$


$-2(-J / N) M \sum_{k_{\beta} k_{i}}\left\langle\phi_{N-k_{\beta}}^{(\downarrow)}, k_{i} \downarrow, k^{\prime} \uparrow ; s_{z} \mid \psi_{k}\right\rangle$

and

$$
2(-J / N) \sqrt{(5+M)(s-M+1)} \sum_{k_{\beta} k_{i}}\left\langle\phi_{N-k_{\beta}}^{(b)}, k_{i} \uparrow, k^{\prime} \uparrow,\left(S_{z}-1\right) \mid \psi_{k}\right\rangle
$$

Hence, the total contribution of the 3 particle intermediate states is,

$$
\begin{aligned}
t_{n f}^{(3)}\left(k, k^{\prime}\right)= & 2(-J / N)\left[M \sum_{k_{\beta} k_{i}}\left\langle\phi_{N-k_{\beta}}^{(\uparrow)}, k_{i} \uparrow, k^{\prime} \uparrow ; s_{z} \mid \psi_{k}\right\rangle\right. \\
& -M \sum_{k_{\beta} k_{i}}\left\langle\phi_{N-k_{\beta}}^{(1)}, k_{i} \downarrow, k^{\prime} \uparrow ; s_{z} \mid \psi_{k}\right\rangle \\
& +\sqrt{(s-M)(s+M+1)} \sum_{k_{\beta} k_{i}}\left\langle\phi_{N-k_{\beta}}^{(\uparrow)}, k_{i} \downarrow, k^{\prime} \uparrow ;\left(s_{z}+1\right) \mid \psi_{k}\right\rangle \\
& \left.+\sqrt{(s+M)(s-M+1)} \sum_{k_{\beta} k_{i}}\left\langle\phi_{N-k_{\beta}}^{(t)}, k_{i} \uparrow, k^{\prime} \uparrow, s_{z}-1\right)\left|\psi_{k}\right\rangle\right]
\end{aligned}
$$

Combining $t_{n f}^{(1)}\left(K K^{\prime}\right)$ and $t_{n f}^{(3)}\left(K, K^{\prime}\right)$ we have the exact scattering equation for spin non-flip scattering as, 


$$
\begin{aligned}
& \left\langle\phi_{N}, k^{\prime} \uparrow, S_{z} \mid \psi_{k}\right\rangle=\frac{i \eta \delta_{k k^{\prime}}}{E_{k}-\left(\varepsilon_{0}+\epsilon_{k^{\prime}}\right)+i \eta}+\frac{(-J / N)}{E_{k}-\left(\varepsilon_{0}+\epsilon_{k^{\prime}}\right)+i \eta} \\
& \int\left\{M \sum_{k^{\prime \prime}}\left\langle\phi_{N}, k^{n} \uparrow, s_{z} \mid \psi_{k}\right\rangle+\sqrt{(s-M)(s+M+1)}\right. \\
& \cdot \sum_{k=1}\left\langle\phi_{N}, k^{\prime \prime} \downarrow_{j}\left(S_{z}+1\right) \mid \psi_{k}\right\rangle \\
& +M\left(u_{q}-n_{t}\right)\left(\phi_{N}, k^{\prime} \uparrow, s_{z}\left|\psi_{k}\right\rangle\right\}
\end{aligned}
$$

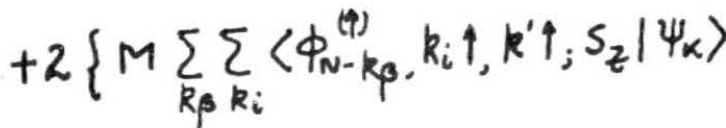

$$
\begin{aligned}
& -M \sum_{k_{\beta}} \sum_{k_{i}}\left\langle\phi_{N-k_{\beta}}^{(\downarrow)}, k_{i} \downarrow, k^{\prime} \varphi_{j} s_{z} \mid \psi_{k}\right\rangle \\
& +\sqrt{(s-M)(s+M+1)} \sum_{k_{\beta} k_{i}}\left\langle\phi_{N-k_{\beta}}^{(\nu)} k_{i} \downarrow, k^{\prime} \uparrow_{j}\left(S_{z}+1\right) \mid \psi_{k}\right\rangle \\
& \left.\left.+\sqrt{(s+M)(s-M+U} \sum_{k_{\beta}} \sum_{k_{i}}\left\langle\phi_{N-k_{p}}^{(\alpha)} \cdot k_{i} \uparrow, k^{\prime} \psi_{j}\left(s_{z}-1\right) \mid \psi_{k}\right\rangle\right\}\right]
\end{aligned}
$$

For spin-flip t-matrix given in (IV. 17), we again have the two contributions arising from single particle and three particle states. Same considerations as for spin non-flip case lead us to the following expressions for these contributions, 


$$
\begin{aligned}
t_{f}^{(U)}\left(k, k^{\prime}\right)=(-J / N)\left\{-(M+1) \sum_{k^{\prime \prime}}\left\langle\phi_{N}, k^{\prime \prime} \downarrow,\left(S_{z}+1\right) \mid \psi_{k}\right\rangle\right. \\
+\sqrt{(s-M)(s+M+1)} \sum_{k^{\prime \prime}}\left\langle\phi_{N}, k^{\prime \prime} \uparrow, s_{z} \mid \psi_{k}\right\rangle \\
\left.+(M+1)\left(n_{\uparrow}-n_{\downarrow}\right)\left\langle\phi_{N}, k^{\prime} \downarrow,\left(s_{z}+1\right) \mid \psi_{k}\right\rangle\right\}
\end{aligned}
$$

Notice that for spin-flip scattering, the single particle contribution comes from the terms $\left(a_{k, \uparrow}^{+} a_{k_{2} \uparrow}-a_{k_{1} \downarrow}^{+} a_{k_{2} \downarrow}\right) s_{z}, a_{k_{1} \downarrow}^{+} a_{k_{2} \uparrow} s_{+}$ of the interaction.

$$
\begin{aligned}
& t_{f}^{(3)}\left(k, k^{\prime}\right)=2(-J / N)\left\{(M+1) \sum_{k_{\beta} k_{i}} \sum_{N-k_{\beta}}\left\langle k_{i}^{(\varphi)} \uparrow, k^{\prime} \psi_{j}\left(S_{z}+1\right) \mid \psi_{k}\right\rangle\right. \\
& -(M+1) \sum_{k_{\beta}} \sum_{k_{i}}\left\langle\phi_{N-k_{\beta}}^{(b)}, k_{i} \downarrow, k^{\prime} \downarrow_{j}\left(S_{e}+1\right) \mid \psi_{k}\right\rangle \\
& +\sqrt{(s+M+2)(s-M+1)} \sum_{k_{\beta} k_{i}} \sum_{\left\langle\phi_{N}(\uparrow)\right.} k_{\beta} \downarrow k_{i}, k^{\prime} \downarrow ;\left(s_{z}+2\right)\left|\psi_{k}\right\rangle \\
& \left.+\sqrt{(s-M)(s+M+1)} \sum_{k_{\beta}} \sum_{k_{i}}\left\langle\phi_{N-k_{\beta}}^{(b)}, k_{i} \uparrow, k^{\prime} \downarrow ; s_{z} \mid \psi_{k}\right\rangle\right\}
\end{aligned}
$$

The first two terms here are the contribution of 3 particle intermediate states for the static part of $\mathrm{H}_{s-\mathrm{d}^{\bullet}}$ The third is the contribution of $a_{k_{1}+}^{+} a_{k_{3} \downarrow} s_{-}$and the last term from $a_{k_{1} \phi}^{+} a_{k_{2} \uparrow} S_{+}$. 
$-46-$

Thus, combining $t_{f}^{(\prime)}\left(k, k^{\prime}\right)$ and $t_{f}^{(k)}\left(k, k^{\prime}\right)$ we have the exact scattering equation for single particle to single particle, spin-flip scattering as,

$$
\begin{aligned}
& \left\langle\phi_{N}, k^{\prime} \downarrow j\left(S_{z}+1\right) \mid \psi_{k}\right\rangle=\frac{(-J / N)}{E_{K}-\left(\varepsilon_{0}+\epsilon_{k^{\prime}}\right)+i \eta} \\
& \cdot\left[\left\{-(M+1) \sum_{k \prime \prime}\left\langle\phi_{N}, k^{\prime \prime} \downarrow ;\left(S_{z}+1\right) \mid \psi_{k}\right\rangle\right.\right. \\
& +\sqrt{(s-M)(s+M+1)} \sum_{k \prime \prime}\left\langle\phi_{N}, k^{\prime \prime} \varphi_{j} S_{z} \mid \psi_{k}\right\rangle \\
& \left.+(m+1)\left(n_{\uparrow}-n_{\downarrow}\right)\left\langle\phi_{n}, k^{\prime} \downarrow ;\left(s_{z}+1\right) \mid \psi_{k}\right\rangle\right\} \\
& +2\left\{( M + 1 ) \sum _ { k _ { \beta } k _ { i } } \sum _ { N - k _ { \beta } } \left(\phi_{i}^{(\uparrow)} \uparrow, k^{\prime} \downarrow ;\left(s_{z}+1\right)\left|\psi_{k}\right\rangle\right.\right. \\
& -(M+1) \sum_{k_{\beta}} \sum_{k_{i}}\left\langle\phi_{N-k_{\beta}}^{(\downarrow)}, k_{i} \downarrow, k^{\prime} \downarrow ;\left(S_{z}+1\right) \mid \psi_{k}\right\rangle \\
& +\sqrt{(s+M+2)(s-M-1)} \sum_{k_{\beta}} \sum_{k_{i}}\left\langle\phi_{N-k_{\beta}}^{(\uparrow)} k_{i} \downarrow, k^{\prime} \downarrow_{j}\left(S_{z}+2\right) \mid \psi_{k}\right\rangle \\
& \left.\left.+\sqrt{(s-M)(s+M+1)} \sum_{k_{\beta}} \sum_{k_{i}}\left\langle\phi_{N-k_{\rho}}^{(\downarrow)} k_{i} \uparrow, k^{\prime} \downarrow ; s_{z} \mid \psi_{k}\right\rangle\right\}\right]
\end{aligned}
$$

(IV. 19) 
Equations (IV. 18) and (IV. 19) form the basic set of coupled integral equations to be solved for single particle initial state to single particle final state, spin non-flip and spin-flip t-matrices. It is also seen explicitly that even single particle t-matrix depends on the higher intermediate states. This clearly shows the many body nature of the present problem, the motion of a single particle depending on the motion of others. 


\section{SOLUTION OF THE SCATTERING EQUATIONS}

One cannot hope to solve the coupled pair of equations (IV. 18) and (IV. 19) for to know the amplitudes in single particle space, we are forced to determine the amplitudes in the three particle space. As an attempt in this direction, we may consider single particle to three particle scattering processes. But such a process would involve, as we shall see in detail later on, $t$-matrices of the form,

$$
\left\langle\phi_{N-k_{\beta}}^{\left(\sigma_{\beta}\right)}, k_{i} \sigma_{i}, k_{j} \sigma_{j} ; S_{z}\left|H_{A-d}\right| \psi_{k}\right\rangle
$$

The introduction of a complete set of intermediate states between $\mathrm{H}_{\mathrm{s}-\mathrm{d}}$ and $\left|\psi_{k}\right\rangle$, shall now bring about contributions from single particle, single particle + one (electron + hole) pair, and single particle + two (electron + hole) pair states. (In our shorter terminology these are single particle, three particle and five particle states, respectively. From now on we shall always use this terminology for brevity). As mentioned earlier, this is because $\mathrm{H}_{\mathrm{s}-\mathrm{d}}$, being bilinear in $\mathrm{a}^{+}$and $\mathrm{a}$, can create and destroy one particle. Thus we see that an attempt to consider amplitudes in three particle space forces us into five particle space. In general any consideration of amplitudes in $(2 m+1)$ particle space shall require knowledge of amplitudes in $(2 m-1)$ and $(2 m+3)$ particle space. 
This dependence of amplitudes in any given number of particle space, forces us to write and solve an infinite system of coupled integral equations, if we desire exact solutions for any amplitude. Clearly, this is impossible and we are forced to make a cutoff in our set of integral equations at some point, with the hope that the contribution of next higher particle space is much smaller, and for even higher number of particle spaces, the contributions converge fast. Such cut-off procedures are to be found everywhere in many body non-trivial problems. In particular, for the present problem of the s-d exchange interaction, Nagaoka's Greens functions technique relies on cutting off at two electron Green's function and Suhl's S-matrix, dispersion theoretic approach relies on considering only the "single"* particle intermediate states. ${ }^{(5)}$

It is our aim, and hope, to be able to consider the higher order contributions and yet be able to provide meaning ful solutions. Up to this point we have derived the two basic, exact, coupled integral equations, (IV. 18) and (IV. 19), describing the single particle to single particle scattering process. Before we get ourselves involved with the basic difficulty of being able to consider higher order contributions in single particle to single particle scattering process, it would be extremely helpful to be able to see the motivation behind our choosing the particular approach we would take in this direction in later sections. This motivation is best brought to light by considering the approximate equations obtained from the exact equations * The correspondence between Suhl's (14) "Single" particle states and our usage of the term "single" is not very clear. 
(IV. 18) and (IV. 19), when the three particle contributions are neglected, and the method of solving the se resulting approximate equations. We devote the next section to this.

A. The Single Particle Intermediate State Approximation

Neglecting the contribution of the three particle intermediate states in equations (IV. 18) and (IV.19), we obtain the following two coupled integral equations describing the scattering from a given single particle state to another single particle state, within the single particle intermediate state approximation.

$$
\begin{aligned}
&\left\langle\phi_{N}, k^{\prime} \uparrow, s_{z} \mid \psi_{k}\right\rangle= \frac{i \eta \delta_{k K^{\prime}}}{E_{k}-\left(\varepsilon_{0}+\epsilon_{k^{\prime}}\right)+i \eta}+\frac{(-J / N)}{E_{k}-\left(\varepsilon_{0}+\epsilon_{k^{\prime}}+i \eta\right)} \\
& \cdot\left[M \sum_{k^{\prime \prime}}\left\langle\phi_{N^{\prime}}, k^{\prime \prime} \uparrow, S_{z} \mid \psi_{k}\right\rangle+\sqrt{(s-M)(s+M+1)}\right. \\
& \sum_{k^{\prime \prime}}\left\langle\phi_{N^{\prime}} k^{\prime \prime} \downarrow_{j}\left(s_{z}+1\right) \mid \psi_{k}\right\rangle+M\left(n_{q}-n_{\downarrow}\right) \\
&\left.\cdot\left\langle\phi_{N}, k^{\prime} \uparrow, S_{z} \mid \psi_{k}\right\rangle\right]
\end{aligned}
$$

and

$$
\begin{aligned}
& \left\langle\phi_{N_{1}} k^{\prime} \downarrow,\left(S_{z}+1\right) \mid \psi_{k}\right\rangle=\frac{(-J / N)}{E_{K}-\left(\varepsilon_{0}+\epsilon_{k^{\prime}}\right)+i \eta}\left[-(M+1) \sum_{K^{\prime \prime}}\left\langle\phi_{N_{1}} k^{\prime \prime} \downarrow ;\left(S_{z}+1\right) \mid \psi_{K}\right\rangle\right. \\
& +\sqrt{(S-M)(s+M+1)} \sum_{k^{\prime \prime}}\left\langle\phi_{N_{1}} k^{m} \varphi_{j} ; S_{z} \mid \psi_{k}\right\rangle \\
& \left.+(M+1)\left(n_{p}-n_{b}\right)\left(\phi_{N}, k^{\prime} \downarrow,\left(\psi_{2}+1\right) \mid \psi_{k}\right)\right]
\end{aligned}
$$


Transferring the terms $M\left(n_{\uparrow}-n_{b}\right)\left\langle\phi_{N}, k^{\prime} \uparrow_{j}, S_{Z} \mid \psi_{k}\right\rangle$ and $(M+1)\left(n_{q}-n_{\downarrow}\right)\left\langle\phi_{N_{1}} k^{\prime},\left(s_{q}+1\right) \mid \psi_{k}\right\rangle$ to the L.H.S. in (V.1) and (V.2), and dividing by the resulting coefficients of $\left.\left|\phi_{N^{\prime}} k^{\prime} \uparrow ; S_{Z}\right| \psi_{k}\right\rangle$ and $\left|\phi_{N^{\prime}} k^{\prime} \downarrow ;\left(S_{Z}+1\right)\right| \psi_{K}>$, we obtain,

$$
\begin{aligned}
\left\langle\phi_{N, k^{\prime} \uparrow, s_{z}\left|\psi_{k}\right\rangle}=\right. & \frac{i \eta \delta_{k k^{\prime}}}{E_{k}-\varepsilon_{0}-\epsilon_{k^{\prime}}-(-J / N) M \cdot \Delta n+i \eta} \\
& +\frac{(-J / N)[M \cdot A+\sqrt{(S-M)(S+M+1)} \cdot B]}{E_{k}-\varepsilon_{0}-\epsilon_{k^{\prime}}-(-J / N) M \cdot \Delta \eta+i \eta}
\end{aligned}
$$

and

$$
\left\langle\phi_{N_{1}} k^{\prime} \downarrow ;\left(S_{z}+1\right) \mid \psi_{K}\right\rangle=\frac{(-J / N)[-(M+1) \cdot B+\sqrt{(S-M)(S+M+1)} \cdot A]}{E_{K}-\varepsilon_{0}-\epsilon_{K^{\prime}}-(-J / N)(M+1) \Delta U+i \eta}
$$

where

$$
\begin{gathered}
\Delta n=\left(n_{q}-n_{l}\right) \\
A=\sum_{x^{\prime \prime}}\left\langle\phi_{N}, k^{\prime \prime} \uparrow, s_{z} \mid \psi_{k}\right\rangle
\end{gathered}
$$


and

$$
\left.B=\sum_{k^{\prime \prime}}\left(\phi_{N}, k^{\prime \prime} \downarrow, \beta_{z}+1\right) \mid \psi_{k}\right)
$$

$\Delta \mathrm{n}$ represents the balance of the spin-up and spin-down electrons. A look at the terms like $(-\mathrm{J} / \mathrm{N}) \mathrm{S}_{\mathrm{Z}} \cdot \Delta \mathrm{n}$ therefore tells us that it represents the molecular field renormalization of the chemical potential and the magnetic moment of the conduction electrons. Energy corrections of this form were encountered long before, by Frohlic and Nabarro ${ }^{(34)}$ in the case of the nucleus in a metal and by Zener ${ }^{(28)}$ in the case of the s-d model. Second order perturbation calculations of the energy were made by Ruderman and Kittel ${ }^{(35)}$, Kasuya ${ }^{(29)}$ and Yosida ${ }^{(36)}$ This leads to the well known $\mathrm{R}$ K K Y result for spin polarization of the conduction electrons. Equations (V.3) and (V.4) from the set of two coupled integral equations to be solved for the spin non-flip and spin-flip $t$-matrices within the approximation of single particle intermediate states. However, the solution of these coupled integral equations in our formulation is trivial if we note that the right hand sides of both the equations have $K^{\prime}$ dependence only in the energy denominators through $\epsilon_{K^{\prime}}$. Hence, summing both the equations over $K^{\prime}$ we shall have on the left hand sides of (V.3) and (V.4) $\sum_{K^{\prime}}\left\langle\phi_{N^{\prime}} k^{\prime} f^{\prime} S_{Z} \mid \Psi_{K}\right\rangle$ and $\sum_{K^{\prime}}\left\langle\phi_{N} k^{\prime} \downarrow ;\left(S_{Z}+1\right) \mid \psi_{K}\right\rangle$ respectively, which are nothing but $A$ and $B$. Thus we obtain the following two simultaneous algebraic equations, 


$$
\begin{aligned}
A= & \sum_{k^{\prime}}\left\{\frac{i \eta \delta_{K k^{\prime}}}{\left\{E_{k}-\varepsilon_{0}-\epsilon_{k^{\prime}}-(-J / N) M \cdot \Delta \eta+i \eta\right\}}\right. \\
+ & (-J / N)\left\{\sum_{k^{\prime}} \frac{1}{\left\{E_{k}-\varepsilon_{0}-\epsilon_{k^{\prime}}-(-J / N) M \Delta \eta+i \eta\right\}}\right\} \\
& {[M \cdot A+\sqrt{(S-M)(S+M+1)} \cdot B] }
\end{aligned}
$$

and,

$$
\begin{aligned}
B=(-J / N) & \left\{\sum_{k^{\prime}} \frac{1}{\left\{E_{k}-\varepsilon_{0}-\epsilon_{k^{\prime}}-(-J / N)(M+1) \Delta n+i \eta\right\}}\right\} \\
& {[-(M+1) B+\sqrt{(S-M)(S+M+1)} \cdot A] }
\end{aligned}
$$

Defining,

$$
X_{1}\left(E_{k}-\varepsilon_{0}\right)=\sum_{k^{\prime}}\left\{E_{k}-\varepsilon_{0}-\epsilon_{k^{\prime}}-(-J / N) M \cdot \Delta n+i \eta\right\}^{-1}
$$

and

$$
X_{2}\left(E_{k}-\varepsilon_{0}\right)=\sum_{k^{\prime}}\left\{E_{k}-\varepsilon_{0}-\epsilon_{k^{\prime}}-(-J / N)(M+1) \cdot \Delta n+i \eta\right\}^{-1}
$$

we have on solving (V.5) and (V.6) 


$$
\begin{gathered}
B=(-J / N) \sqrt{(S-M)(S+M+1)} X_{2}\left(E_{K}-\varepsilon_{0}\right) \\
{\left[1+(-J / N)(M+1) X_{2}\left(E_{K}-\varepsilon_{0}\right)\right]^{-1} \cdot A} \\
A=\left[1-(-J / N) M X_{1}\left(E_{K}-\varepsilon_{0}\right)-(-J / N)^{2}(S-M)(S+M+1) X_{1}\left(E_{K}-\varepsilon_{0}\right)\right. \\
\left.\cdot X_{2}\left(E_{K}-\varepsilon_{0}\right) \cdot\left\{1+(-J / N)(M+1) X_{2}\left(E_{K}-\varepsilon_{0}\right)\right\}^{-1}\right]^{-1}
\end{gathered}
$$

Knowing these closed form solutions for $A$ and $B$ we have from (V. 3) and (V. 4),

$$
\begin{aligned}
t_{h f}^{(1)}= & (-J / N)\left\{M+(-J / N) s(s+1) X_{2}\left(E_{k}-\varepsilon_{0}\right)\right\} \\
& \cdot\left[1-(-J / N)\left\{M X_{1}\left(E_{k}-\varepsilon_{0}\right)-(M+1) X_{2}\left(E_{k}-\varepsilon_{0}\right)\right\}\right. \\
& \left.-(-J / N)^{2} s(s+1) X_{1}\left(E_{k}-\varepsilon_{0}\right) \cdot X_{2}\left(E_{k}-\varepsilon_{0}\right)\right]^{-1}
\end{aligned}
$$

and, 


$$
\begin{gathered}
t_{f}^{(1)}=(-J / N) \sqrt{(S-M)(S+M+1)} \cdot\left[1-(-J / N)\left\{M X_{1}\left(E_{k}-\varepsilon_{0}\right)\right.\right. \\
\left.-(M+1) X_{2}\left(E_{k}-\varepsilon_{0}\right)\right\}-(-J / N)^{2} s(S+1) \\
\left.\cdot X_{1}\left(E_{k}-\varepsilon_{0}\right) \cdot X_{2}\left(E_{k}-\varepsilon_{0}\right)\right]^{-1}
\end{gathered}
$$

(V. 9 and (V.10) provide us with the $\mathrm{t}$-matrices in the single particle intermediate states approximation. We could discuss the nature of these solutions, but it would be better to discuss the se after we have the solutions for which the higher intermediate states have also been considered. We can then make a direct comparison of the two and see how the inclusion of spin life time effects alter the nature of the solutions. However, it is perhaps important to point out that the Kondo type anomaly already shows up in our solutions (V.9) and (V.10), because of energy sums like $X$, which for continuous spectrum (or in other words in the thermodynamic limit of volume and the number of particles $\mathrm{N}$, tending to infinity) become

$$
\begin{aligned}
(-J) \cdot \sum_{k^{\prime}}\left\{E_{k}-\varepsilon_{0}-\epsilon_{k^{\prime}}\right. & -(-J / N) M \cdot \Delta n+i \eta\}^{-1} \\
& \longrightarrow(-J) \int \frac{\rho\left(\epsilon_{k^{\prime}}\right)\left\{1-2 f\left(\epsilon_{k^{\prime}}\right)\right\} d \epsilon_{k^{\prime}}}{\left\{E_{k^{\prime}}-\varepsilon_{0}-\epsilon_{k^{\prime}}-(-J) M \Delta n+i \eta\right\}}
\end{aligned}
$$


where, $\rho\left(\epsilon_{k^{\prime}}\right)$ is the single particle density of states per electron, $f\left(\epsilon_{k^{\prime}}\right)=\left\{\exp \left(\epsilon_{k^{\prime}} / k_{B} T\right)+1\right\}^{-1} \quad$, is the Fermi distribution function and $\Delta x \quad$ refers to difference in number of spinup and spin-down electrons, per electron.

Such integrals show the well known logarithmic singularities and consequently, a perturbation expansion in powers of $\mathrm{J}$ of our results (V.9) and (V.10) show these singularities as $\mathrm{J}^{\mathrm{m}} \ln ^{\mathrm{n}} \mathrm{T}$, $m=1--(n-1)$, and agree with the results obtained by perturbation techniques. But our results (V.9) and (V.10) already provide the correct behavior at $T=0$ and Fermi energy, since we have a higher power $\mathcal{X}$ sum in the denominator. As a consequence of this we shall see that our t-matrices are well behaved at $\mathrm{T}=0$ and Fermienergy. We shall also find that the blowing up behavior of perturbation theory (at $\mathrm{T}=0$ and Fermi energy) is replaced by the occurrence of resonances, signifying the onset of some interesting phenomenon, which we shall discuss later on.

To summarize, we have seen in this section on solution of the scattering equations in single particle intermediate state approximation, that a simple summation over the single particle energies $\epsilon_{k}$, reduced the problem of solving two coupled integral equations into triviai problem of solving two simultaneous algebraic equations. Thus, the equations obtained by Abrikosov and Doniach by their diagram summation procedures and their subsequent solutions, are obtained here almost trivially and the results are already correct to logarithmic accuracy. Our aim from now on therefore, is to 
exploit this summation technique of reducing integral equations into algebraic equations when we consider scattering to higher intermediate state $\mathrm{s}$ in the next section.

It is perhaps worthwhile to mention at this stage that our summation technique is valid for a more general class of interactions than the contact interaction being considered here. In general, for a momentum dependent exchange coupling $\mathrm{J}\left(\mathrm{K}, \mathrm{K}^{\prime}\right)$ which can be approximated in the mean by a finite sum over factorisable interactions as,

$$
J\left(k, k^{\prime}\right)=\sum_{i=1}^{m} R_{i}(k) S_{i}\left(k^{\prime}\right)
$$

our technique shall still work. This is so because in our formalism the interaction coupling, $J\left(K, K^{\prime}\right)$ becomes the kernel of the integral equations and the form (V.11) of $J\left(K, K^{\prime}\right)$ reduces the kernel to a degenerate kernel. The exchange coupling $\mathrm{J}\left(\mathrm{K}, \mathrm{K}^{\prime}\right)$ can be approximated in the "mean" if given $\delta>0$, these exists a set of $2 \mathrm{~m}$ functions

$$
\begin{aligned}
& \left\{R_{i}(K), S_{i}(K)\right\}_{i=1}^{m} \text {, such that, } \\
& \quad \int \frac{d k d k^{\prime}}{(2 \pi)^{3}}\left|J\left(k, k^{\prime}\right)-\sum_{i=1}^{m} R_{i}(k) S_{i}\left(k^{\prime}\right)\right|<\delta
\end{aligned}
$$


B. Contribution of Multiparticle Intermediate States

Returning to the exact scattering equations (IV. 18) and (IV. 19), we realize that to obtain a solution for them, we have to somehow evaluate three particle contributions. We therefore consider the one to three particle scattering processes. We recall that our initial single particle state was $\left|\phi_{\mathrm{N}}, \mathrm{k}^{\uparrow} ; \mathrm{S}_{\mathrm{Z}}\right\rangle$. In the final three particle states, we have eight possible states corresponding to the four possible ways in which the electron-hole pair can be excited each for spin non-flip and spin flip scattering of the single particle $K^{\prime}$. Explicitly, these four possibilities are e.g., for spin non-flip scattering of $\mathrm{K}^{\prime \uparrow}$, $\left|\phi_{N-k_{\beta}}^{(\uparrow)}, k_{m} \uparrow, k^{\prime} \uparrow ; s_{z}\right\rangle,\left|\phi_{N-k_{\beta}}^{(\uparrow)}, k_{m} \downarrow, k^{\prime} \uparrow,\left(s_{z}+1\right)\right\rangle,\left|\phi_{N-k_{\beta}}^{(b)}, k_{m} \uparrow, k^{\prime} \uparrow_{j}\left(s_{z}-1\right)\right\rangle$ and $\left|\phi_{N-k_{p}}^{(t)} k_{m} \downarrow, k^{\prime} \uparrow ; s_{z}\right\rangle$. Similar four possibilities

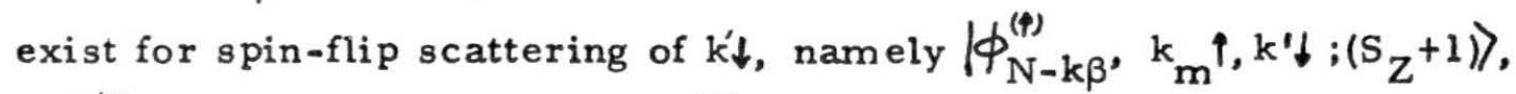

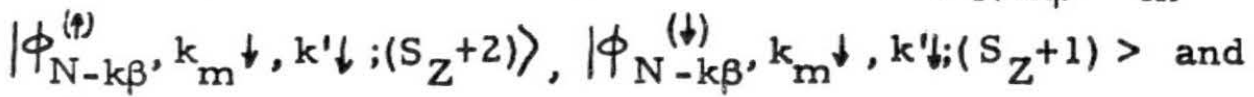
$\left|\phi_{\mathrm{N}-\mathrm{k} \beta}^{(\downarrow)}, \mathrm{k}_{\mathrm{m}}^{\uparrow, \mathrm{k}^{\prime} \downarrow ; \mathrm{S}} \mathrm{Z}\right\rangle$.

Let us consider the case where the three particle state is $\mid \phi_{N-k \beta}^{(\uparrow)}, k_{m}^{\uparrow}, k^{\prime} \downarrow,\left(S_{Z}+1\right)>$. Going back to our basic scattering equation (IV.8), we have upon taking the three particle projection of $\left|\Psi_{k}\right\rangle$, the following equation,

$$
\left\langle\phi_{N-k_{\beta}}^{(\uparrow)}, k_{m} \uparrow, k^{\prime} \uparrow ; s_{z} \mid \psi_{k}\right\rangle=\frac{i \eta\left\langle\phi_{N-k_{\beta}}^{(\uparrow)}, k_{m} \uparrow, k^{\prime} \uparrow, s_{z} \mid \phi_{N}, k \uparrow ; s_{z}\right\rangle}{E_{k}-\left(\varepsilon_{0}+\epsilon_{k^{\prime}}+\epsilon_{k_{m}}-\epsilon_{k_{\beta}}\right)+i \eta}
$$




$$
\frac{\left\langle\phi_{N-k_{\beta}}^{(\uparrow)}, k_{m} \uparrow, k^{\prime} \uparrow ; s_{z}\left|H_{s-\alpha}\right| \phi_{k}\right\rangle}{E_{k}-\left(\varepsilon_{0}+\epsilon_{k^{\prime}}+\epsilon_{k_{m}}-\epsilon_{k_{\beta}}\right)+i \eta}
$$

Because of the orthogonality of the single and three particle wave functions, the first term on the R. H.S. is zero. This is, of course, true of any scattering process where the number of particles is not the same in the initial and final states. Thus all single particle to higher number of particle state scattering equations shall be homogeneous.

$$
\text { Now, consider the quantity }\left\langle\phi_{N-k_{\beta}}^{(\mathscr{e}}, k_{m} \uparrow, k^{\prime} \uparrow ; s_{z}\left|\mathcal{H}_{\beta-\alpha}\right| \psi_{\kappa}\right\rangle
$$

which is the t-matrix element in three particle space. If, as in the case of t-matrix in single particle space, we introduce a complete set of eigenstates between $\mathrm{H}_{s-d}$ and $\left|\psi_{k}\right\rangle$, we find that the bilinear character of the interaction $\mathrm{H}_{\mathrm{s}-\mathrm{d}}$, explained in detail earlier, shows up by giving non-vanishing contributions for single, three and five particle intermediate states only. Considering the general three particle $t$-matrix element

we have,

$$
\left\langle\phi_{N-k_{\beta}}^{\left(\sigma_{\beta}\right)}, k_{m} \sigma_{m}, k_{m} \sigma_{m}, s_{z}\left|\mathcal{H}_{A-\alpha}\right| \psi_{K}\right\rangle
$$


$-60-$

$$
\begin{aligned}
& \left\langle\phi_{N-k_{\beta}}^{\left(\sigma_{\beta}\right)} k_{m} \sigma_{m}, k_{n} \sigma_{n}, s_{z}|\mathcal{H}|_{\alpha-\alpha} \mid \psi_{k}\right\rangle \\
& =\left[\sum_{k^{\prime \prime} r^{\prime \prime} S_{z}^{\prime \prime}}\left\langle\phi_{N-k \rho}^{\left(\sigma_{\rho}\right)}, k_{m} \sigma_{m}, k_{n} \sigma_{n}, S_{z}\left|H_{\Delta-\alpha}\right| \phi_{N}, k^{\prime \prime} \sigma_{j}^{\prime \prime} S_{z}^{\prime \prime}\right\rangle\right. \\
& \left\langle\phi_{N}, k^{\prime \prime} \sigma^{\prime \prime}, S_{z} " \mid \psi_{k}\right\rangle \\
& +\sum \sum \sum \sum\left\langle\phi_{N-k_{\beta}}^{\left(\sigma_{\beta}\right)}, k_{m} \sigma_{m}, k_{n} \sigma_{n}, S_{z}\left|H_{\Delta-d}\right| \phi_{N-k_{\alpha}}^{\left(\sigma_{\alpha}\right)}, k_{i} \sigma_{i}, k_{j} \sigma_{j} ; S_{z}^{\prime \prime}\right\rangle \\
& k_{\alpha} \sigma_{\alpha} k_{i} \sigma_{i} k_{j} \sigma_{j} s_{z}^{\prime \prime} \\
& \left\langle\phi_{N-k_{\alpha}}^{\left(\sigma_{\alpha}\right)}, k_{i} \nabla_{i}, k_{j} \sigma_{j} ; s_{z}^{n} \mid \psi_{k}\right\rangle \\
& +2 \sum \sum \sum \sum \sum\left\langle\phi_{N-k_{\beta}}^{\left(\sigma_{\beta}\right)} k_{m} k_{m} \sigma_{m}, k_{n} \sigma_{n} ; s_{z}\left|H_{\Delta-d}\right|\right. \\
& k_{\alpha,}, \sigma_{\alpha}, k_{\alpha_{2}} \sigma_{\alpha} k_{i} r_{i} k_{j} \sigma_{j} \cdot k_{p} \sigma_{p} S_{z} " \\
& \left|\phi_{N-k_{\alpha_{1}} k_{\alpha_{2}}}^{\left(\sigma_{\alpha_{1}}\right)\left(\sigma_{\alpha_{2}}\right)}, k_{i} \sigma_{i}, k_{j} \sigma_{j}, k_{p} \sigma_{p}, S_{z}^{\prime \prime}\right\rangle
\end{aligned}
$$

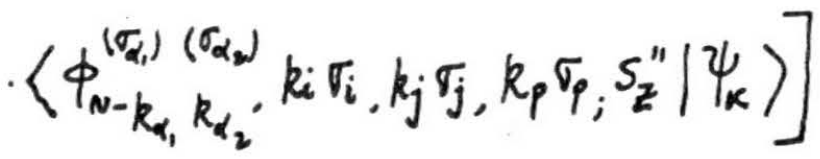

Thus we see that the consideration of three particle space contribution has forced us into the five particle space. We therefore at this stage again make a cut-off at the three particle contribution. This shall make the problem consistent in the three particle space. We are neglecting the five particle contributions in one to three particle scattering processes only for the time being, and because this enables us to use our summation technique to again obtain a closed set of algebraic equations from which we can evaluate the three par- 
ticle projections. Substitution of these three particle contributions in terms of the single particle matrix elements in our exact scattering equations (IV. 18) and (IV. 19) shall provide us with a closed pair of equations in single particle space, which can again be solved by our summation technique.

In short then, we shall show in this section that the neglect of five particle space contributions in one to three particle scattering process, enables us to evaluate the three particle projection sums of the type occurring in our exact single particle to single particle scattering equations (IV. 18) and (IV.19) in terms of the single particle projections. Then later on we generalize this mathematical structure of the contribution of higher particle space to all higher order intermediate states.

Returning to equation (V.13), we have on evaluating $\left.<\phi_{\mathrm{N}-\mathrm{k} \beta}^{(\uparrow)}, \mathrm{k}_{\mathrm{m}} \uparrow, \mathrm{k}^{\prime} \uparrow ; \mathrm{S}_{\mathrm{Z}}\left|\mathrm{H}_{\mathrm{s}-\mathrm{d}}\right| \psi_{\mathrm{K}}\right)$ in the standard way as done before for single particle t-matrix elements, the following equation,

$$
\begin{gathered}
\left\langle\phi_{N-k_{p}}^{(\uparrow)}, k_{m} \uparrow, k^{\prime} \uparrow, s_{z} \mid \psi_{k}\right\rangle=\frac{(-J / N)}{E_{k}-\left(\varepsilon_{0}+\epsilon_{k^{\prime}}+\epsilon_{k_{m}}-\epsilon_{k_{\beta}}\right)+i \eta} \\
{\left[\left\{M\left\langle\phi_{N}, k^{\prime} \uparrow, s_{z} \mid \psi_{k}\right\rangle-M\left\langle\phi_{N}, k_{m} \uparrow ; s_{z} \mid \psi_{k}\right\rangle\right\}\right.} \\
+M \Delta n\left\langle\phi_{N-k_{\beta}}^{(\uparrow)}, k_{m} \uparrow, k^{\prime} \uparrow ; s_{z} \mid \psi_{k}\right\rangle
\end{gathered}
$$




$$
\begin{aligned}
+M\left\{\sum_{k_{j}}\left\langle\phi_{N-k_{\beta}}^{(\uparrow)}, k_{j} \uparrow, k^{\prime} \uparrow, s_{z} \mid \psi_{k}\right\rangle\right. \\
\left.\quad-\sum_{k_{j}}\left\langle\phi_{N-k_{\rho}}^{(\uparrow)}, k_{j} \uparrow, k_{m} \uparrow, s_{z} \mid \psi_{k}\right\rangle\right\} \\
+\sqrt{(5-M)(s+M+1)}\left\{\sum_{k_{j}}\left\langle\phi_{N-k_{\beta}}^{(\uparrow)}, k_{j} \downarrow, k^{\prime} \uparrow_{j}\left(S_{z}+1\right)\right| \psi_{k}\right) \\
\left.\left.-\sum_{k_{j}}\left\langle\phi_{N-k_{p}}^{(\uparrow)}, k_{j} \downarrow, k_{m} \uparrow_{j}\left(S_{z}+1\right) \mid \psi_{k}\right\rangle\right\}\right]
\end{aligned}
$$

We have two sums in each curly bracket on the R.H.S. corresponding to the direct and exchange terms. It is also seen immediately that for $k_{m}=k^{\prime}$ the R.H.S. becomes inentically zero, as is required of the L. H.S. because of the anti-symmetry of the wave function.

Similar considerations provide the following equations for the other possible three particle projections

$$
\begin{aligned}
& \left\langle\phi_{N-k_{\beta}}^{(\uparrow)}, k_{m} \downarrow, k^{\prime} \uparrow ;\left(s_{z}+1\right) \mid \psi_{k}\right\rangle=\frac{(-J / N)}{E_{k}-\left(\varepsilon_{0}+\epsilon_{k}+\epsilon_{k_{m}}-\epsilon_{k_{\beta}}\right)+i \eta} \\
& {\left[\left\{-(M+1)\left\langle\phi_{N}, k_{m} \downarrow ;\left(s_{z}+1\right)\right| \psi_{k}\right)+\sqrt{(s-M)(S+M+1)}\left(\phi_{N_{1}} k^{\prime} \uparrow, s_{z} \mid \psi_{k}\right)\right\}}
\end{aligned}
$$


$-63-$

$$
\begin{aligned}
& +(M+1) \Delta n\left\langle\phi_{N-k_{p}}^{(\uparrow)}, k_{m} \downarrow, k^{\prime} \uparrow ;\left(s_{z}+1\right) \mid \psi_{k}\right\rangle \\
& +(M+1)\left\{\sum_{k_{j}}\left\langle\phi_{N-k_{\beta}}^{(\uparrow)}, k_{m} \downarrow, k_{j}\right\}_{j},\left(\zeta_{z}+1\right)\left|\psi_{k}\right\rangle\right. \\
& \left.-\sum_{k_{j}}\left\langle\phi_{N-k_{\beta}}^{(\uparrow)}, k_{j} \downarrow, k^{\prime} \uparrow,\left(S_{z}+1\right) \mid \psi_{k}\right\rangle\right\} \\
& \left.+\sqrt{(s+M+2)(s-M-1)}\left\{\sum_{k_{j}}\left\langle\phi_{N-k_{p}}^{(j)}, k_{j} \downarrow, k_{m} t_{j},\left(s_{z}+2\right)\right| \psi_{k}\right)\right\} \\
& \left.+\sqrt{(s-M)(s+M+1)}\left\{\sum_{k_{j}}\left\langle\phi_{N-k_{p}}^{(q)}, k_{j} \uparrow, k^{\prime} \uparrow ; S_{z} \mid \psi_{k}\right\rangle\right\}\right]
\end{aligned}
$$

$$
\begin{aligned}
& \left\langle\phi_{N-k_{\beta}}^{(b)}, k_{m} \uparrow, k^{\prime} \uparrow_{j}\left(s_{z}-1\right) \psi_{k}\right\rangle=\frac{(-J / N)}{E_{k}-\left(\varepsilon_{0}+\epsilon_{K^{\prime}}+\epsilon_{k_{m}}-\epsilon_{k_{\beta}}\right)+i \eta} \\
& {\left[\left\{\sqrt{(s+M)(s-M+1)}\left\{\left\langle\phi_{N}, k^{\prime} \uparrow ; s_{z} \mid \psi_{k}\right\rangle-\left\langle\phi_{N}, k_{m} \uparrow ; s_{z} \mid \psi_{k}\right\rangle\right\}\right.\right.} \\
& +(m-1) \Delta n\left\langle\phi_{N-k_{\beta}}^{(k)}, k_{m} \uparrow, k^{\prime} \eta,\left(s_{z}-1\right) \mid \psi_{k}\right\rangle \\
& +(M-1)\left\{\sum_{k_{j}}\left\langle\phi_{N-k_{p}}^{(1)}, k_{j} \uparrow, k^{\prime} \uparrow_{j},\left(S_{z}-1\right) \mid \psi_{k}\right\rangle-\sum\left\langle\phi_{k_{j}}^{(())}, k_{p}, k_{j} \eta_{j} k_{m} \uparrow_{j}\left(S_{z}-1\right) \mid \psi_{k}\right\rangle\right\}
\end{aligned}
$$


$-64-$

$$
\begin{gathered}
+\sqrt{(S+M)(s-M+1)}\left\{\sum_{k_{j}}\left\langle\phi_{N-k_{\beta}}^{(\downarrow)}, k_{j} \downarrow, k^{\prime} \uparrow ; S_{z} \mid \psi_{k}\right\rangle\right. \\
\left.\left.-\sum_{k_{j}}\left\langle\phi_{N-k_{\beta}}^{(\downarrow)}, k_{j} \downarrow, k_{m} \uparrow ; S_{z} \mid \psi_{k}\right\rangle\right\}\right]
\end{gathered}
$$

$$
\begin{aligned}
& \left\langle\phi_{N-k \beta}^{(b)}, k_{m} \phi_{1}, k^{\prime} \psi, s_{z} \mid \psi_{k}\right\rangle=\frac{(-J / N)}{E_{k}-\left(\varepsilon_{0}+\epsilon_{k^{\prime}}+\epsilon_{k_{m}}-\epsilon_{k_{\beta}}\right)+i \eta} \\
& {\left[\left\{-M\left(\phi_{,}, k^{\prime} \hat{f}_{j} s_{z} \mid \psi_{K}\right)-\sqrt{(s-M)(s+M+1)}\left\langle\phi_{N}, k_{m} \psi_{j}\left(s_{z}+1\right) \mid \psi_{K}\right\rangle\right\}\right.} \\
& +M \cdot \Delta n\left\langle\phi_{N, k_{\beta}}^{(b)}, k_{m} \downarrow \cdot k^{\prime} \uparrow_{;}, s_{z} \mid \psi_{k}\right\rangle
\end{aligned}
$$

$$
\begin{aligned}
& -M\left\{\sum_{k_{j}}\left\langle\phi_{M-k_{\beta}}^{(t)}, k_{j \downarrow}, k^{\prime} t ; s_{z} \mid \psi_{k}\right\rangle-\sum_{k_{j}}\left\langle\phi_{M-k_{\beta}}^{(t)}, k_{j} \downarrow, k_{m} t_{j}, s_{z} / \psi_{k}\right\rangle\right\} \\
& \left.+\sqrt{(s+M)(s-M+1)} \sum_{k_{j}}\left\langle\phi_{N-k_{\beta}}^{(b)}, k_{j} \uparrow, k^{\prime} t_{j}\left(s_{z}-1\right)\right| \psi_{k}\right) \\
& \left.-\sqrt{(s-M)(s+M+1)} \sum_{k_{j}}\left\langle\phi_{N-k_{p}}^{(s)} k_{j} \downarrow, k_{m} \psi_{j}\left(S_{z}+1\right) \mid \psi_{k}\right\rangle\right]
\end{aligned}
$$


$-65-$

$$
\begin{aligned}
& \left\langle\phi_{N-k_{\beta}}^{(\uparrow)}, k_{m} \uparrow, k^{\prime} \downarrow_{j}\left(S_{z}+1\right) \mid \psi_{k}\right\rangle=\frac{(-J / N)}{E_{k}-\left(\varepsilon_{0}+\epsilon_{k^{\prime}}+\epsilon_{k_{m}}-\epsilon_{k_{\beta}}\right)+i \eta} \\
& {\left[\left\{M+U\left\langle\phi_{N} k^{\prime} \downarrow ;\left(s_{z}+1\right) \mid \psi_{k}\right\rangle-\sqrt{(s-M)(s+M+1)}\left\langle\phi_{N}, k_{m} i_{;} s_{z} / \psi_{k}\right\rangle\right\}\right.} \\
& +(M+1) \Delta n\left\langle\phi_{N-k_{\beta}}^{(\uparrow)}, k_{m} \uparrow, k^{\prime} \psi ;\left(S_{z}+1\right) / \psi_{k}\right\rangle \\
& +(M+1)\left\{\sum_{k_{j}}\left\langle\phi_{N-k_{\beta}}^{(\uparrow)}, k_{j} \uparrow, k^{\prime} \psi_{j}\left(S_{z}+1\right) \mid \psi_{k}\right\rangle\right. \\
& \left.\left.-\sum_{k_{j}}\left\langle\phi_{N-k_{\beta}}^{(\uparrow)}, k_{m} \uparrow, k_{j} \downarrow_{j}\left(S_{z}+1\right)\right| \psi_{k}\right)\right\} \\
& +\sqrt{(s+M+2)(s-M-1)} \sum_{k_{j}}\left\langle\phi_{N \cdot k_{\rho}}^{(\varphi)}, k_{j} \downarrow, k^{\prime} \downarrow ;\left(S_{z}+2\right) / \psi_{k}\right\rangle \\
& \left.+\sqrt{(s-M)(s+M+1)} \sum_{k_{j}}\left\langle\phi_{n \cdot k_{\beta}}^{(\uparrow)}, k_{m} \uparrow, k_{j} \psi_{j}, S_{z} \mid \psi_{k}\right\rangle\right]
\end{aligned}
$$

$$
\begin{aligned}
& \left\langle\phi_{N-k_{\beta}}^{(q)}, k_{m} t_{0} k^{\prime} \psi_{j}\left(s_{z}+2\right) / \psi_{k}\right\rangle=\frac{(-J / N)}{E_{k}-\left(\varepsilon_{0}+\epsilon_{K^{\prime}}+\epsilon_{k_{m}}-\epsilon_{k_{\beta}}\right)+i \eta} \\
& {\left[\sqrt{(s+M+2)(s-M-1)}\left\{\left\langle\phi_{N}, k^{\prime} \psi_{j}\left(s_{z}+1\right) \mid \psi_{k}\right\rangle-\left\langle\phi_{N_{1}} k_{m} b_{j}\left(s_{z}+1\right) \mid \psi_{k}\right\rangle\right\}\right.}
\end{aligned}
$$


$-66-$

$$
\begin{aligned}
& +(M+2) \cdot \Delta u\left\langle\phi_{N-k_{\beta}}^{(\tau)}, k_{m} \downarrow, k^{\prime} \psi_{j}\left(\zeta_{z}+2\right) \mid \psi_{k}\right\rangle \\
& -(M+2)\left\{\sum_{k_{j}}\left\langle\phi_{N_{-} k_{\beta}}^{(\uparrow)} k_{j} \downarrow, k^{\prime} v_{j}\left(S_{z}+2\right) / \psi_{\alpha}\right)\right. \\
& \left.-\sum_{k_{j}}\left\langle\phi_{N-k_{\beta}}^{(\uparrow)}, k_{j} \downarrow, k_{m} \psi_{j}\left(s_{z}+2\right) / \psi_{k}\right)\right\} \\
& +\sqrt{(s+M+2)(s-M-1)}\left\{\sum_{k_{j}}\left\langle\phi_{N-k_{\beta}}^{(1)}, k_{j} \uparrow, k^{\prime} \psi_{j}\left(s_{z}+1\right)\right| \psi_{k}\right) \\
& \left.\left.\left.-\sum_{k_{j}}\left\langle\phi_{N-k_{\rho}}^{(\uparrow)}, k_{j} \uparrow, k_{m} \downarrow,\left(s_{z}+1\right)\right| \psi_{k}\right)\right\}\right]
\end{aligned}
$$

$$
\begin{aligned}
& \left\langle\phi_{N-k_{\beta}}^{(\phi)}, k_{m} t, k^{\prime} \psi_{j}\left(\xi_{z}+1\right) \mid \psi_{k}\right\rangle=\frac{(-J / N)}{E_{k}-\left(\epsilon_{0}+\sigma_{k^{\prime}}+\epsilon_{k_{m}}-\epsilon_{k \beta}\right)+i \eta} \\
& {\left[-(M+1)\left\{\left\langle\phi_{n}, k^{\prime} \psi_{j}\left(S_{z}+1\right) \mid \psi_{k}\right\rangle-\left\langle\phi_{n}, k_{m} \downarrow ;\left(S_{z}+1\right) \mid \psi_{k}\right\rangle\right\}\right.} \\
& +(M+1) \Delta u\left\langle\phi_{N-k_{\beta}}^{(\phi)}, k_{m} \downarrow, k^{\prime} v_{j}\left(S_{z}+1\right) / \psi_{k}\right\rangle \\
& +(M+1)\left\{\sum_{k_{j}}\left\langle\phi_{N-k_{\beta}}^{(\phi)}, k_{j} \downarrow, k_{m} \downarrow_{j}\left(s_{z}+1\right) \mid \psi_{k}\right\rangle\right. \\
& \left.-\sum_{k_{j}}\left\langle\phi_{N-k_{\beta}}^{(\downarrow)}, k_{j} \downarrow k^{\prime} l ;\left(s_{z}+1\right) \mid \psi_{k}\right\rangle\right\} \\
& +\sqrt{(s-M)(s+M+1)}\left\{\sum_{k_{j}}\left\langle\phi_{N-k_{\beta}}^{(\phi)}, k_{j} \uparrow, k^{\prime}\right\rangle, s_{z}\left|\psi_{k}\right\rangle\right. \\
& \left.\left.-\sum_{k_{j}}\left\langle\phi_{N-k_{\beta}}^{(t)}, k_{j} \uparrow, k_{m} k_{j} s_{z} / \psi_{k}\right\rangle\right\}\right]
\end{aligned}
$$


$-67-$

$$
\begin{aligned}
& \left\langle\phi_{N-k_{\beta}}^{\left({ }^{\prime}\right)}, k_{m} t_{1}, k^{\prime} \psi_{j} s_{z} \mid \psi_{k}\right\rangle=\frac{(-J / N)}{E_{k}-\left(\varepsilon_{0}+\epsilon_{k^{\prime}}+\epsilon_{k_{m}}-\epsilon_{k \beta}\right)+i \eta} \\
& {\left[\left\{\sqrt{(S-M)(S+M+1)}\left\langle\phi_{N}, k^{\prime} \downarrow ;\left(S_{z}+1\right) \mid \psi_{K}\right\rangle\right.\right.} \\
& +M\left\langle\phi_{N}, k_{m} \uparrow_{j} s_{z} / \psi_{k}\right\rangle^{+}+M \cdot \Delta n\left\langle\phi_{N-k_{p}}^{(b)}, k_{m} \uparrow, k^{\prime} v_{j}, s_{z} \mid \psi_{k}\right\rangle \\
& +M\left\{\sum_{k_{j}}\left\langle\phi_{N-k_{\beta}}^{(t)}, k_{j} \uparrow, k^{\prime} \downarrow_{j}, s_{z} \mid \psi_{k}\right\rangle\right. \\
& -\sum_{k_{j}}\left\langle\phi_{N-k_{p}}^{(b)}, k_{2 n}\left\{, k_{j} l_{j} s_{z}\left|\psi_{k}\right\rangle\right\}\right. \\
& +\sqrt{(s-M)(s+M+1)} \sum_{k_{j}}\left\langle\phi_{N-k_{\beta}}^{(t)}, k_{j} \downarrow, k^{\prime} \downarrow_{j}\left(k_{z}+1\right) \mid \psi_{k}\right\rangle \\
& \left.+\sqrt{(S+M)(s-M+1)} \sum_{k_{j}}\left\langle\phi_{N-k_{\beta}}^{(d)}, k_{m} \uparrow, k_{j} \downarrow_{j}\left(S_{z}-1\right) \mid \psi_{k}\right\rangle\right]
\end{aligned}
$$

Equations (V. 14) to (V.17) characterize the three particle projections when the state $k^{\prime q}$ is always occupied by one of the electrons while equations (V.18) to (V.21) characterize the situation where the state $k^{\prime}+$ is always occupied by one of the electrons. 
The pair of equations (IV.18) and (IV.19) along with the set of the eight three particle scattering equations (V.14) to (V.21) form a closed set of ten coupled integral equations which describe our scattering process cor rectly within the approximation of retaining terms only up to three particles in the intermediate states.

The occurrence of ten coupled integral equations within the three particle intermediate states seems to pose a formidable problem in any attempt to solve this system of equations. However, our summation technique of reducing the se integral equations into algebraic equations comes in very handy and considerably simplifies the mathematical nature of the problem. Solution of the resulting system of simultaneous algebraic equations presents no complications, although the process is somewhat tedious. As we shall see shortly, fortunately we do not have to even consider the entire system of simultaneous algebraic equations explicitly, for by considering just a suitable pair of the se equations we can show a certain mathematical structure of the nature of three particle contributions to single particle scattering equations namely (IV.18) and (IV.19). This mathematical structure is all we need to realize to consider the contribution of higher particle intermediate states.

To see this simplifying feature of the higher particle contributions, let us consider equations (V.14) and (V.15) describing one to three particle scattering processes, where we have neglected the contribution of five particle intermediate state $s$ in such scattering processes as mentioned before. Transferring the diagonal terms 
$-69-$

$M \cdot \Delta n\left\langle\phi_{N-k_{\beta}}^{(\uparrow)}, k_{m} \uparrow, k^{\prime} \uparrow ; S_{z} \mid \psi_{k}\right\rangle \quad$ and $(M+1) \Delta n\left\langle\phi_{N-k_{\beta}}^{(\uparrow)}, k_{m} \downarrow, k^{\prime} \uparrow\right.$; $\left(s_{z}+1\right)\left|\psi_{k}\right\rangle$ to the L.H.S. of the respective equations, dividing by the resulting coefficients of the two three particle projections involved on the L.H.S. namely $\left\langle\phi_{N-k_{\beta}}^{(\uparrow)}, k_{m} \tau_{,}, k^{\prime} \varphi_{j}, s_{z} \mid \psi_{k}\right\rangle$ and $\left\langle\phi_{N-k_{\beta}}^{(p)}, k_{m} \downarrow, k^{\prime} \tau_{j}\left(s_{\tau}+1\right) / \psi_{k}\right\rangle \quad$ and then summing the resulting equations over $\mathrm{k}_{\mathrm{m}}$, we obtain the following two equations,

$$
\begin{aligned}
& \sum_{k_{m}}\left\langle\phi_{N-k_{\beta}}^{(\uparrow)}, k_{m} \uparrow, k^{\prime} \uparrow_{j} S_{z} \mid \psi_{k}\right\rangle \\
& =(-J / N)\left\{\sum_{k_{m}}\left\{E_{k}-\left(\varepsilon_{0}+\epsilon_{k^{\prime}}+\epsilon_{k_{m}}-\epsilon_{k_{\beta}}\right)-(-J / N) M \cdot \Delta n+i \eta\right\}^{-1}\right. \\
& \cdot\left[M\left\langle\phi_{N}, k^{\prime} \uparrow ; S_{z} \mid \psi_{k}\right\rangle+M \sum_{k_{j}}\left\langle\phi_{N-k_{\beta}}^{(\uparrow)}, k_{j}\right\}, k^{\prime} \varphi_{j} s_{z}\left|\psi_{k}\right\rangle\right. \\
& \left.+\sqrt{(S-M)(S+M+1)} \sum_{k_{j}}\left\langle\phi_{N-k_{\beta}}^{(*)}, k_{j} \downarrow, k^{\prime} \eta_{j}\left(s_{z}+1\right) \mid \psi_{k}\right\rangle\right] \\
& +\sum_{k_{m}}\left[\left(\frac{J}{N}\right)\left\{E_{k}-\left(\varepsilon_{0}+\sigma_{k^{\prime}}+\epsilon_{k_{m}}-\epsilon_{K_{\beta}}\right)-(-J / N) M \cdot \Delta u+i \eta\right\}^{-1}\right. \\
& \cdot\left\{-M\left(\phi_{N_{1}} k_{m}\right\rangle_{j} S_{z}\left|\psi_{N}\right\rangle-M \sum_{k_{j}}\left\langle\phi_{N-k_{\beta}}^{(\uparrow)}, k_{j} \uparrow, k_{m} \uparrow, S_{z} \mid \psi_{k}\right\rangle\right. \\
& \left.\left.\left.-\sqrt{(s-M)(s+M+1)} \sum_{k_{j}}\left\langle\phi_{N-k_{j}}^{(p)}, k_{j} \downarrow,\left.k_{\operatorname{mon}}\right|_{j}\left(s_{e}+1\right)\right| \psi_{x}\right)\right\}\right]
\end{aligned}
$$


$-70-$

and

$$
\begin{aligned}
& \sum_{k_{m}}\left\langle\phi_{N-k_{\beta}}^{(\uparrow)}, k_{m} \downarrow, k^{\prime} \uparrow_{j}\left(S_{z}+1\right) \mid \psi_{k}\right\rangle \\
& =(-J / N)\left\{\sum_{k_{m}}\left\{E_{k}-\left(\varepsilon_{0}+\epsilon_{K^{\prime}}+\epsilon_{k_{m}}-\epsilon_{K_{\rho}}\right)-(-J / N)(M+1) \Delta n+i \eta\right\}^{-1}\right] \\
& ; \sqrt{(S-M)(S+M+1)}\left\langle\phi_{N_{1}} k^{\prime} \uparrow_{j} S_{z} / \psi_{K}\right\rangle-(M+1) \\
& \sum_{k_{j}}\left\langle\phi_{N-k_{\beta}}^{(\uparrow)} \cdot k_{j} \downarrow, k^{\prime} \psi_{j}\left(S_{z}+1\right) / \psi_{k}\right\rangle \\
& \left.+\sqrt{(s-M)(S+M+1)} \sum_{k_{j}}\left\langle\phi_{N \cdot k_{\beta}}^{(\uparrow)}, k_{j} \uparrow, k^{\prime} \eta_{j} S_{z} \mid \psi_{k}\right\rangle\right\} \\
& +\sum_{k_{m}}\left[(-J / N)\left\{E_{k}-\left(\varepsilon_{0}+\epsilon_{k^{\prime}}+\epsilon_{k_{m}}-\epsilon_{k_{\beta}}\right)-\left(-\frac{J}{\pi}\right)(m+1) \Delta n+i \eta\right\}^{-1}\right. \\
& \left\{-(M+1)\left\langle\phi_{N}, k_{m} \downarrow ;\left(S_{z}+1\right) / \psi_{k}\right)\right. \\
& +(M+1) \sum_{k_{j}}\left\langle\phi_{N-k_{\beta}}^{(\omega)}, k_{m} \downarrow, k_{j} \uparrow,\left(5_{z}+1\right) \mid \psi_{k}\right\rangle \\
& \left.\left.\left.+\sqrt{(s+M+2)(s-M-1)} \sum_{k_{j}}\left\langle\phi_{N-k_{\beta}}^{(p)}, k_{m} \downarrow, k_{j} \downarrow ;\left(\beta_{z}+2\right)\right| \psi_{k}\right)\right\}\right]
\end{aligned}
$$

The terms in the second square brackets of equations (V.22) and (V.23) can be looked upon as the contribution of the exchange terms because of the antisymmetric nature of the many electron wave functions. The presence of these terms does not allow these equations to become algebraic equations as simply as we saw in the case of single particle intermediate states in the previous section. However, we note that in these terms, since they involve a summation 
-71 .

over $\mathrm{km}$, the only free indices left are $E_{\mathrm{K}^{\prime}}, \epsilon_{\mathrm{K}^{\prime}}$ and $\epsilon_{\mathrm{K}_{\beta}}$. Thus we define two functions $F_{1}\left(E_{K^{\prime}} \epsilon_{K^{\prime}}, \epsilon_{K \beta}\right)$ ard $F_{2}\left(E_{K^{\prime}}, \epsilon_{K^{\prime}}, \epsilon_{K_{\beta}}\right)$ as,

$$
\begin{aligned}
& F_{1}\left(E_{k}, \epsilon_{k^{\prime}}, \epsilon_{k_{\beta}}\right) \cdot\left\langle\phi_{N,} k^{\prime} \uparrow, s_{z} \mid \psi_{k}\right\rangle \\
& =\sum_{k_{m}}\left[(-J / N)\left\{E_{k}-\left(\varepsilon_{0}+\epsilon_{k^{\prime}}+\epsilon_{k_{m}}-\epsilon_{k \rho}\right)-(-J / N) M \cdot \Delta n+i \eta\right\}^{-1}\right. \\
& \cdot\left\{-M\left\langle\phi_{N}, k_{m} \uparrow, S_{z} \mid \psi_{k}\right\rangle-M \sum_{k_{j}}\left\langle\phi_{N-k_{\beta}}^{(\uparrow)}, k_{j} \uparrow, k_{m} \uparrow_{j} S_{z} \mid \psi_{k}\right\rangle\right. \\
& \left.\left.-\sqrt{(S-M)(S+M+1)} \sum_{k_{j}}\left\langle\phi_{N-R_{\beta}}^{(\uparrow)}, k_{j} \downarrow, k_{m} i_{j}\left(S_{z}+1\right) \mid \psi_{k}\right\rangle\right\}\right]
\end{aligned}
$$

and,

$$
\begin{aligned}
& F_{2}\left(E_{k}, \epsilon_{k}, \epsilon_{k \beta}\right) \cdot\left\langle\phi_{N}, k^{\prime} \uparrow, s_{z} \mid \psi_{k}\right\rangle \\
& =\sum_{k_{m}}\left[(-J / N)\left\{E_{k}-\left(\varepsilon_{0}+\epsilon_{k^{\prime}}+\epsilon_{k_{m}}-\epsilon_{k_{\beta}}\right)-(-J / N)(M+1) \Delta u+i \eta\right\}^{-1}\right. \\
& \cdot\left\{-(M+1)\left\langle\phi_{N} k_{m} \downarrow_{j}\left(S_{z}+1\right) / \psi_{k}\right\rangle+(M+1)\right. \\
& \sum_{k_{j}}\left\langle\phi_{N-k_{\beta}}^{(\uparrow)}, k_{m} t, k_{j} \uparrow_{j}\left(S_{z}+1\right) \mid \psi_{K}\right\rangle \\
& \left.\left.+\sqrt{(s+M+2)(s-M-1)} \sum_{k_{j}}\left\langle\phi_{N-k_{\beta}}^{(\mu)}, k_{m} \downarrow, k_{j} \downarrow ;\left(s_{z}+2\right) \mid \psi_{k}\right\rangle\right\}\right]
\end{aligned}
$$

Thus equations (V.22) and (V.23) become, 
$-72$.

$$
\begin{aligned}
& \sum_{k_{m}}\left\langle\phi_{N-k_{p}}^{(\uparrow)}, k_{m} \uparrow, k^{\prime} \uparrow ; s_{z} \mid \psi_{k}\right\rangle \\
& =x_{3}\left(E_{k}, \epsilon_{k^{\prime}}, \epsilon_{k_{\beta}}\right)\left[\left\{M\left\langle\phi_{N^{\prime}}, k^{\prime} \uparrow_{j} S_{z}\right| \psi_{k}\right)\right. \\
& +M \sum_{k_{j}}\left\langle\phi_{N-k_{\beta}}^{(t)}, k_{j} \uparrow, k^{\prime} \uparrow_{j} s_{z} \mid \psi_{k}\right\rangle \\
& \left.\left.\left.+\sqrt{(S-M)(S+M+1)} \sum_{k_{j}}\left\langle\phi_{N-k_{\beta}}^{(t)}, k_{j} \downarrow, k^{\prime} \varphi_{j}\left(S_{z}+1\right)\right| \psi_{k}\right)\right\}\right] \\
& +F_{1}\left(E_{k}, \epsilon_{K^{\prime},} \epsilon_{k \beta}\right)\left\langle\phi_{N}, k^{\prime} \uparrow, s_{z} \mid \psi_{k}\right\rangle
\end{aligned}
$$

and

$$
\begin{aligned}
& \sum_{k_{m}}\left\langle\phi_{N-k_{\beta}}^{(\uparrow)}, k_{m} \downarrow, k^{\prime} \uparrow_{j}\left(S_{z}+1\right) \mid \psi_{k}\right\rangle \\
& =X_{4}\left(E_{k}, \epsilon_{k^{\prime}}, \epsilon_{k_{\beta}}\right)\left[\sqrt { ( s - M ) ( s + M + 1 ) } \left\{\left\langle\phi_{w}, k^{\prime} \eta_{j}, s_{z} / \psi_{k}\right)\right.\right. \\
& \left.+\sum_{k_{j}}\left\langle\phi_{N-k_{p}}^{(\uparrow)}, k_{j} \uparrow, k^{\prime} \uparrow_{j} s_{z} \mid \psi_{k}\right\rangle\right\} \\
& \left.\left.-(M+1) \sum_{k_{j}}\left\langle\phi_{N-k_{\beta}}^{(\uparrow)}, k_{j} \downarrow, k^{\prime} \uparrow_{j}\left(S_{z}+1\right)\right| \psi_{k}\right)\right] \\
& +F_{2}\left(E_{k}, \epsilon_{k^{\prime}}, \epsilon_{k_{\beta}}\right) \cdot\left\langle\phi_{N}, k^{\prime} \uparrow, S_{z} \mid \psi_{k}\right\rangle
\end{aligned}
$$

where

$$
\begin{aligned}
& X_{3}\left(E_{k,} \epsilon_{k^{\prime}}, \epsilon_{k_{\beta}}\right) \\
= & \sum\left\{E_{k}-\left(\varepsilon_{0}+\epsilon_{k^{\prime}}+\epsilon_{k_{m}}-\epsilon_{k_{\beta}}\right)-(-J / N) M \cdot \Delta n+i \eta\right\}^{-1} \cdot(-J / N)
\end{aligned}
$$


and

$$
\begin{aligned}
& X_{4}\left(E_{k}, \epsilon_{k^{\prime}}, \epsilon_{k_{\beta}}\right) \\
& =(-J / N) \sum_{k_{m}}\left\{E_{k}-\left(\varepsilon_{0}+\epsilon_{k^{\prime}}+\epsilon_{k_{m}}-\epsilon_{k_{\beta}}\right)-(-J / N)(M+1) \cdot \Delta n+i \eta\right\}^{-1}
\end{aligned}
$$

Now we can solve the algebraic equations (V.26) and (V.27) for the sums $\quad \sum_{k_{m}}\left\langle\phi_{N-k_{\beta}}^{(\uparrow)}, k_{m} \uparrow, k^{\prime} t_{j} s_{z} \mid \psi_{k}\right\rangle \quad$ and $\sum_{k_{m}}\left\langle\phi_{N-k_{p}}^{(p)}\right.$, $k_{m} w, k^{\prime} \uparrow_{j}\left(s_{z}+1\right)\left|\psi_{k}\right\rangle$ in terms of the single particle projection $\left\langle\phi_{N}, k^{\prime} \phi_{j} s_{\mathbb{2}} / \psi_{k}\right\rangle$ and the functions $F_{1}\left(\varepsilon_{k}, \epsilon_{k^{\prime}}, \epsilon_{k_{\beta}}\right)$ and $f_{2}\left(E_{k}, \epsilon_{k^{\prime}}, \epsilon_{k p}\right)$. We obtain the following re sults,

$$
\begin{aligned}
& \sum_{k_{m}}\left\langle\phi_{N-k_{\beta}}^{(\uparrow)}, k_{m} \uparrow, k^{\prime} \uparrow_{j} s_{z} \mid \psi_{k}\right\rangle \\
& =\left[\sqrt{(S-M)(s+M+1)} \cdot X_{4}\left\{1+M X_{3} \cdot \alpha_{1}^{-1}\right\} \cdot \beta_{1}^{-1} \cdot\left\langle\phi_{N_{1}} k^{\prime} \gamma_{j} s_{2} \mid \psi_{k}\right\rangle\right. \\
& +\beta_{1}^{-1}\left\{\sqrt{(s-M)(s+M+1)} x_{4} \cdot \alpha_{1}^{-1} \cdot F_{1}\left(E_{k,}, \epsilon_{k^{\prime}}, \epsilon_{k \beta}\right)\left\langle\phi_{N_{1}} k^{\prime} \tau_{j}, s_{z} \mid \psi_{k}\right\rangle\right. \\
& \left.\left.+F_{2}\left(E_{k}, G_{k}, \epsilon_{k p}\right) \cdot\left(\phi_{n,} k^{\prime} \eta, s_{z} \mid \psi_{k}\right)\right\}\right]
\end{aligned}
$$




$$
\begin{aligned}
& \sum_{k_{m}}\left\langle\phi_{N-k \beta}^{(\uparrow)}, k_{m} \downarrow, k^{\prime} \uparrow,\left(S_{z}+1\right) \mid \psi_{k}\right\rangle \\
& =\left[\left\{M X_{3}+(s-M)(s+M+1) X_{3} X_{4}\left(1+M x_{3} \cdot \alpha_{1}^{-1}\right) \cdot \beta_{1}^{-1}\right\}\right. \\
& \cdot \alpha_{1}^{-1}\left\langle\phi_{N}, k^{\prime} \phi_{j} s_{z} / \psi_{k}\right)+\left\{1+(s-M)(s+M+1) x_{3} x_{4}\right. \\
& \left.\cdot \alpha_{1}^{-1} \beta_{1}^{-1}\right\} \alpha_{1}^{-1} f_{1}\left(E_{k}, G_{k^{\prime}}, \epsilon_{k}\right)\left(\phi_{n}, k^{\prime} \tau_{j}, s_{z} / \psi_{k}\right) \\
& +\sqrt{(s-M)(s+M+1)} x_{3} \cdot \alpha_{1}^{-1} \cdot \beta_{1}^{-1} f_{2}\left(\varepsilon_{k}, \epsilon_{k^{\prime}}, \epsilon_{k \beta}\right) \\
& \left.\cdot\left\langle\phi_{N} k^{\prime} \uparrow ; s_{z} \mid \psi_{k}\right\rangle\right]
\end{aligned}
$$

where

$$
\alpha_{1}=\left[1-M X_{3}\left(E_{k}, G_{k^{\prime}}, \epsilon_{k \beta}\right)\right]
$$

and

$$
\begin{aligned}
& \beta_{1}=\left[1+(M+1) X_{4}\left(E_{k}, \epsilon_{k^{\prime}}, \epsilon_{k \beta}\right)-(s-M)(s+M+1)\right.
\end{aligned}
$$

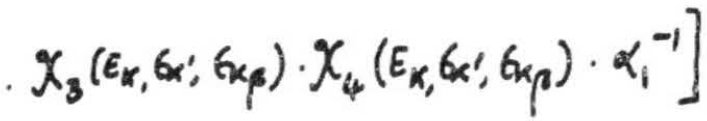


observe that the three particle projection sums, $\sum_{k_{m}}\left\langle\phi_{N-k_{p}}^{(\uparrow)}, k_{m} \phi^{\prime}\right.$, $k^{\prime} f_{j} s_{z}\left|\psi_{k}\right\rangle$ and $\sum_{k_{m}}\left\langle\phi_{N_{0}}^{\left(\phi_{k}\right)}, k_{m} \phi_{j}, k^{\prime} \varphi_{j}\left(s_{z}+1\right) \mid \psi_{k}\right\rangle$ are of the form $A_{1}\left(E_{x}, \epsilon_{x^{\prime}}, \epsilon_{x_{\beta}}\right)\left\langle\phi_{N}, k^{\prime} \varphi, s_{*} / \psi_{k}\right) \quad$ and

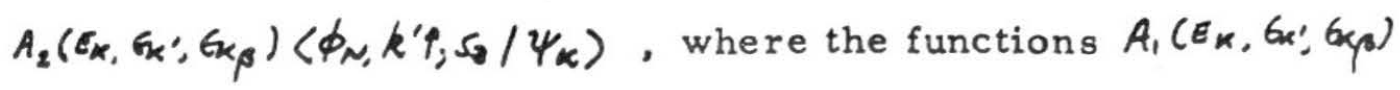

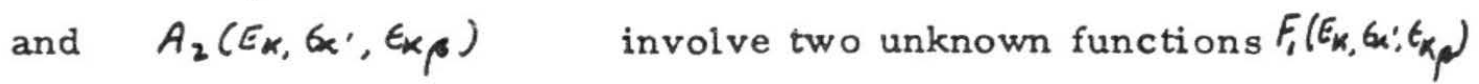
and $F_{2}\left(E_{x}, G_{x} ; E_{k \beta}\right)$. As was pointed out earlier, the functions $F_{1}$ and $F_{2}$ in effect give the contribution of exchange terms. The contribution of direct terms is explicitly given in terms of the functions $x_{3}\left(E_{\alpha}, \epsilon_{\alpha^{\prime}}, \epsilon_{\alpha \beta}\right)$ and $x_{4}\left(E_{\alpha}, \epsilon_{\alpha}: \epsilon_{\alpha \beta}\right)$ which can be evaluated. A lack of knowledge of the functions $F_{1}$ and $F_{2}$ does not present any difficulty for our purposes, for our aim was to show the fact that the contribution of the three particle projection sums of the form (V.28) is simply some function of the energies $\boldsymbol{E}_{k}, \boldsymbol{G}_{k^{\prime}}$ and $\epsilon_{\kappa_{\beta}}$ times the single particle amplitude $\left\langle\phi_{N} k^{\prime} \phi_{j} s_{z} \mid \psi_{k}\right\rangle$. This mathematical structure is sufficient to simplify the problem tremendously by allowing us to eliminate the three particle contributions from our single particle to single particle scattering equations (IV. 18) and (IV. 19).

At this point it seems important to point out that the mathematical structure of the three particle projection sums that we have shown just now, could have been seen to be the case by simply looking at the nature of the three particle projection sums occurring in our exact single particle to single particle scattering equations (IV.18) and (IV.19). Returning to the se equations, we notice that the. three particle sums involved correspond to processes where one of the electrons goes unscattered, while a particle + hole pair is created 
by the action of the interaction. For example, the sum, $\sum_{k_{m}} \sum_{k_{\beta}}\left\langle\phi_{N-k \beta}^{(f)}\right.$, $k_{j} \uparrow, k^{\prime} \varphi_{;} s_{z}\left|\psi_{k}\right\rangle$ refers to the sum over all those intermediate states where one of the particles is already in the final state $K ' \uparrow$ (i.e. has gone unscattered) while the effect of the interaction has been to excite a particle + hole pair. It is a well known result in field theoretic scattering formulations that the contribution of such processes is always proportional to the scattering amplitude. More explicitly, considering the situation in terms of diagrams, we have schematically, for a process of three particles coming in and three going out for instance

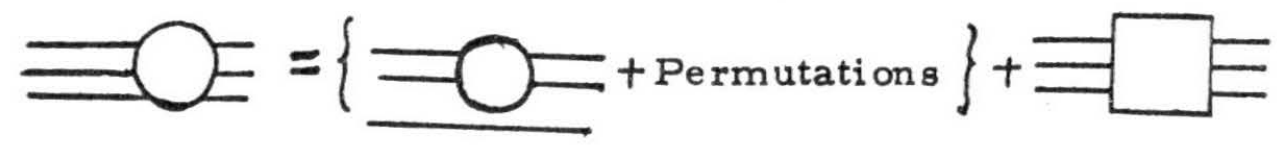

where the first part on the right hand side is called the "Disconnected" part and the second the "Connected" part, in the conventional terminology of diagrammatic techniques. In this language then, we have the well known result that the "Disconnected" part (i.e., where one particle goes unscattered) is proportional to a scattering amplitude. Hence it also follows that this part is not really an analytic function of the energies etc. Because of the particular nature of our interaction $\mathrm{H}_{\mathrm{s}-\mathrm{d}}$ we find that the three particle projection sums involved are only of this form. We shall later on see that their contribution is not analytic. (For further discussion of this point, see ref. (37)). Consideration of equations (V.14) and (V.15) led us to show explicitly that the three particle projection sums being considered can be written as, 


$$
\begin{aligned}
& \sum_{k_{m}}\left\langle\phi_{N-k_{p}}^{(\uparrow)}, k_{m} \uparrow, k^{\prime} \uparrow ; S_{z} \mid \psi_{k}\right\rangle \\
& =A_{1}\left(E_{k}, \epsilon_{x^{\prime}}, \epsilon_{k \beta}\right)\left(\phi_{N_{1}} k^{\prime} \uparrow_{j} s_{z} / \psi_{k}\right) \\
& \sum_{k_{m}}\left\langle\phi_{N-k_{p}}^{\left(q_{j}\right)} k_{m} \downarrow, k^{\prime} \eta_{j}\left(S_{z}+1\right) \mid \psi_{k}\right\rangle \\
& =A_{2}\left(E_{k}, G_{x^{\prime}}, \sigma_{x_{\beta}}\right) \cdot\left\langle\phi_{N_{1}} k^{\prime} \varphi_{j} S_{z} / \psi_{k}\right)
\end{aligned}
$$

Returning to our single particle to single particle scattering equations (IV. 18) and (IV. 19) we notice that we need to know three particle projection sums of the kind, $\sum_{k_{m}} \sum_{k_{\beta}}\left\langle\phi_{N-k_{\rho}}^{(\varphi)}, k_{m} \uparrow, k^{\prime} \tau_{j} s_{z} \mid \psi_{k}\right\rangle$ etc. Thus from expressions (V.30) and (V.31) we find that,

$$
\begin{aligned}
& \sum_{k_{\beta}} \sum_{k_{m}}\left\langle\phi_{N-k_{\beta}}^{(\uparrow)} \cdot k_{m} t_{1}, k^{\prime} \tau_{j}, s_{z} / \psi_{k}\right)=\sum_{k_{\beta}} A_{1}\left(E_{k}, \sigma_{x^{\prime}}, \epsilon_{k \beta}\right) \\
& \text { - }\left\langle\phi_{N}, k^{\prime} q_{j} s_{z} / \psi_{k}\right\rangle \\
& \begin{aligned}
& \sum_{k_{\beta}} \sum_{k_{m}}\left\langle\phi_{N-k_{\beta}}^{(\uparrow)}, k_{m} t, k^{\prime} \eta_{j}\left(S_{z}+1\right) \mid \psi_{k}\right\rangle= \sum_{k_{\beta}} A_{2}\left(\epsilon_{k}, \sigma_{k^{\prime}}, \epsilon_{k_{\beta}}\right) \\
& \cdot\left\langle\phi_{N}, k^{\prime} \uparrow ; s_{z} \mid \psi_{k}\right\rangle
\end{aligned}
\end{aligned}
$$

Defining the functions $A_{1}\left(E_{K^{\prime}}, \epsilon_{K^{\prime}}\right)$ and $A_{2}\left(E_{K^{\prime}}, \epsilon_{K^{\prime}}\right)$ by

$$
\begin{aligned}
& \sum_{k_{\beta}} A_{1}\left(E_{k}, \epsilon_{k^{\prime}}, \epsilon_{k \beta}\right)=A_{1}\left(E_{k^{\prime}}, \epsilon_{k^{\prime}}\right) \\
& \sum_{k_{\beta}} A_{2}\left(E_{k}, \epsilon_{k^{\prime}}, \epsilon_{k \beta}\right)=A_{2}\left(E_{k}, \epsilon_{k^{\prime}}\right)
\end{aligned}
$$

respectively, we finally

find that the three particle projection sums in equations (IV.18) and (IV. 19) have the general structure, 


$$
\begin{aligned}
& \sum_{k_{\beta} k_{m}}\left\langle\phi_{N-k_{\beta}}^{(\uparrow)}, k_{m} \uparrow, k^{\prime} \uparrow_{j} S_{z} \mid \psi_{k}\right\rangle=A_{1}\left(E_{k^{\prime}}, \epsilon_{k^{\prime}}\right)\left\langle\phi_{N}, k^{\prime} \uparrow_{j} S_{z} \mid \psi_{k}\right\rangle \\
& \sum_{k_{\beta} k_{m}}\left\langle\phi_{N-k_{\beta}}^{(\uparrow)}, k_{m} \downarrow, k^{\prime} \uparrow,\left(S_{z}+1\right) \mid \psi_{k}\right\rangle=A_{2}\left(\varepsilon_{\kappa}, \epsilon_{k^{\prime}}\right)\left\langle\phi_{N}, k^{\prime} \varphi_{j} S_{z} \mid \psi_{k}\right\rangle
\end{aligned}
$$

It is clear that similar considerations of the pairs of equations (V.16) and (V.17), (V.18) and (V.19), and (V.20) and (V.21) shall yield the other three particle projection sums involved in the single particle to single particle spin non-flip and spin-flip scattering equations (IV. 18) and (IV. 19). Thus without repeating the detailed analysis for these pairs of equations, we simply state that the structure of the remaining two three particle projection sums occurring in (IV. 18) is of the form,

$$
\begin{aligned}
& \sum_{k_{\beta} k_{m}}\left\langle\phi_{N-k_{\beta}}^{(t)}, k_{m} \uparrow, k^{\prime} \uparrow,\left(s_{z}-1\right) \mid \psi_{k}\right\rangle=A_{3}\left(E_{k}, \epsilon_{k^{\prime}}\right)\left\langle\phi_{N}, k^{\prime} \uparrow_{j} s_{z} \mid \psi_{k}\right\rangle \\
& \sum_{k_{p} k_{m}}\left\langle\phi_{N-k_{\beta}}^{(t)}, k_{m} \downarrow, k^{\prime} \uparrow, s_{z} \mid \psi_{k}\right\rangle=A_{4}\left(E_{k}, \epsilon_{k}\right)\left\langle\phi_{N}, k^{\prime} \uparrow_{j} s_{z} \mid \psi_{k}\right\rangle
\end{aligned}
$$

The three particle projection sums occurring in the spin-flip scattering equation (IV. 19) are found, from (V. 18) and (V. 19), and (V.20) and (V.21), to be of the form, 


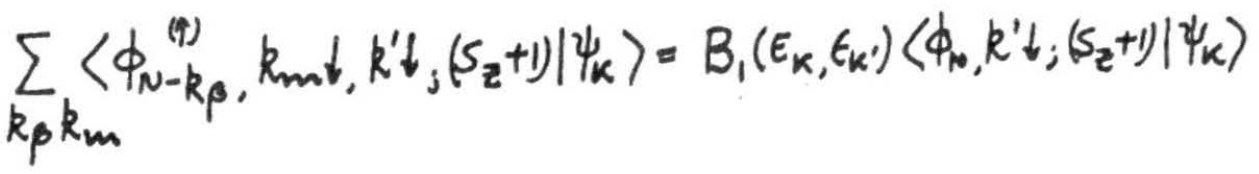

$$
\begin{aligned}
& \sum_{k_{\beta} k_{m}}\left\langle\phi_{N-k_{p}}^{(\uparrow)}, k_{m} \downarrow, k^{\prime} \downarrow_{j}\left(\xi_{z}+2\right) \mid \psi_{k}\right\rangle=B_{2}\left(E_{k}, \epsilon_{k^{\prime}}\right)\left\langle\phi_{N}, k^{\prime} \downarrow_{j}\left(S_{z}+1\right) \mid \psi_{k}\right\rangle \\
& \sum_{k_{\beta} k_{m}}\left\langle\phi_{N-k_{p}}^{(b)}, k_{m} \downarrow, k^{\prime} l_{j}\left(S_{z}+1\right) \mid \psi_{k}\right\rangle=B_{3}\left(E_{k_{1}}, \epsilon_{k^{\prime}}\right)\left\langle\phi_{N^{\prime}}, k^{\prime} l^{\prime},\left(\left\langle_{z}+1\right)\left|\psi_{k}\right\rangle\right.\right. \\
& \sum_{k_{\beta} k_{m}}\left\langle\phi_{N-k_{\beta}}^{\left({ }^{\prime}\right)}, k_{m} \uparrow, k^{\prime} \downarrow_{j}, s_{z} \mid \psi_{k}\right\rangle=B_{4}\left(E_{k}, \epsilon_{k^{\prime}}\right)\left\langle\phi_{N}, k^{\prime} \downarrow_{j}\left(\zeta_{z}+1\right) \mid \psi_{k}\right\rangle
\end{aligned}
$$

Thus far in this section, we have succeeded in showing the general mathematical structure of the three particle projection sums involved in our exact scattering equations (IV. 18) and (IV. 19), by resorting to a cut off procedure at the three particle intermediate states in writing down our equations for one to three particle scattering process. Thus it may appear that this mathematical structure of the contribution of three particle intermediate states in single particle to single particle scattering equations is true within the cut of procedure used in writing down higher scattering processes, namely, neglect of five and higher particle scattering processes. However, a careful thought on the analysis involved in arriving at this nature of contribution of the three particle projection sums within the approximation for which we have shown it explicitly, 
immediately tells us that this analysis can be generalized to any higher order with our conclusion remaining the same. Also, instead of doing the same analysis for higher order scattering process involved, we may at this point make use of the well known result in high energy scattering theory quoted earlier which tells us that the mathematical structure at which we have arrived here, within our cut off procedure, remains valid in general. Let us therefore at this point make the ansatz that the nature of contribution of the three particle projection sums involved in our single particle to single particle scattering equation (IV. 18), and (IV.19) is of the form,

$$
\begin{aligned}
\sum_{k_{\beta} k_{m}}\left\langle\phi_{N-k_{\beta}}^{\left(\sigma_{\beta}\right)}, k_{m} \sigma_{m}, k^{\prime} \sigma^{\prime \prime} s_{z}^{\prime \prime} \mid \psi_{k}\right\rangle \\
=A\left(E_{k}, \epsilon_{k}\right)\left\langle\phi_{N}, k^{\prime} \sigma^{\prime} ; s_{z}^{\prime} \mid \psi_{k}\right\rangle
\end{aligned}
$$

such that $\left(S_{z}+\uparrow\right)=\left(s_{z}^{\prime}+\sigma^{\prime}\right)=\left(S_{z}^{\prime \prime}+\sigma^{\prime \prime}+\sigma_{m}+\bar{\sigma}_{\beta}\right)$ so that spin conservation is satisfied.

Even though the use of the theorem on the "Disconnected" part tells us that our ansatz is in fact correct to all orders, for an explicit exposition of this fact, we generalize our result to all orders in Appendix 1.

C. Solution of the Exact Single Particle Scattering Equations.

In the beginning of the previous section we stated the motivation for considering the mathematical nature of the contribution of the three particle projection sums involved in the two, single 
particle initial state to single particle final state, spin non-flip and spin-flip scattering equations (IV. 18) and (IV. 19). Having shown that this mathematical structure is given to all orders by (V. 32), we are now in a position to incorporate the effect of all higher order intermediate states in single particle scattering processes. With the help of (V. 32), we replace the three particle contributions in (IV. 18)i and (IV. 19) by $\lambda_{1}\left(E_{k}, \epsilon_{k^{\prime}}\right)\left(\phi_{N_{1}} k^{\prime} \varphi_{j} S_{z} / \psi_{k}\right)$ and $\lambda_{2}\left(E_{k}, \epsilon_{k^{\prime}}\right)\left\langle\phi_{N}, k^{\prime} \downarrow_{j}\left(s_{z}+U / \psi_{k}\right)\right.$ respectively, to obtain,

$$
\begin{aligned}
& \left\langle\phi_{n}, k^{\prime} \varphi_{j} s_{z} \mid \psi_{k}\right\rangle=i \eta \delta_{k k^{\prime}} \cdot\left\{E_{k}-\left(\varepsilon_{0}+\epsilon_{k^{\prime}}\right)+i \eta\right\}^{-1} \\
& +(-J / N)\left\{E_{K}-\left(\varepsilon_{0}+\epsilon_{K^{\prime}}\right)+i \eta\right\}^{-1} \cdot\left[\left\{M \Delta u\left(\phi_{N}, k^{\prime} \uparrow_{j} s_{z} \mid \psi_{k}\right)\right.\right. \\
& +M \sum_{k^{\prime \prime}}\left\langle\phi_{N}, k^{n} \eta_{j} s_{z} \mid \psi_{k}\right\rangle+\sqrt{(s-M)(s+M+1)} \\
& \left.\sum_{k^{\prime \prime}}\left\langle\phi_{N}, k^{n} \downarrow ;\left(s_{z}+1\right) \mid \psi_{k}\right\rangle\right\} \\
& \left.+\lambda_{1}\left(E_{k}, \epsilon_{k^{\prime}}\right)\left\langle\phi_{N}, k^{\prime} \uparrow_{j} s_{z} \mid \psi_{k}\right\rangle\right]
\end{aligned}
$$

and, 


$$
\begin{aligned}
& \left\langle\phi_{N^{\prime}} k^{\prime} \downarrow_{j}\left(S_{z}+1\right) \mid \psi_{k}\right\rangle \\
& =(-J / N)\left\{E_{k}-\left(\varepsilon_{0}+\epsilon_{k^{\prime}}\right)+i \eta\right\}^{-1} \\
& \quad\left[\left\{(M+1) \Delta n\left\langle\phi_{N}, k^{\prime} \downarrow_{j} s_{z^{\prime}} \mid\right\rangle-(M+1)\right.\right. \\
& \left.\sum_{k^{*}}\left\langle\phi_{N^{\prime}} k^{\prime \prime} \downarrow_{j}\left(S_{z}+1\right)\right| \psi_{k}\right)+\sqrt{(s-M)(s+M+1)} \\
& \left.\cdot \sum_{k^{\prime \prime}}\left\langle\phi_{N^{\prime}} k^{\prime \prime} \uparrow_{j} s_{z} \mid \psi_{k}\right\rangle\right\} \\
& \left.+\lambda_{2}\left(E_{k^{\prime}}, \epsilon_{k^{\prime}}\right)\left\langle\phi_{N}, k^{\prime} \downarrow_{j}\left(s_{z}+1\right) \mid \psi_{k}\right\rangle\right]
\end{aligned}
$$

The functions $\lambda_{1}\left(E_{k}, G_{k}\right)$ and $\lambda_{2}\left(E_{k}, E_{k}\right)$ are of course unknown at this point. However, we shall soon obtain equations to determine them by exploiting self-consistent boundary conditions to be satisfied by our scattering amplitudes. These functions have been introduced to take into account the effect of higher order intermediate states. In other words, $\lambda_{1}\left(\varepsilon_{k_{k}}, \epsilon_{\kappa^{\prime}}\right)$ and $\lambda_{2}\left(E_{k}, G_{k^{\prime}}\right)$ take into account the contributions of (particle + hole) pair excitations, due to spontaneous spin fluctuations of the impurity atom in spin non-flip and spin-flip scattering processes of the conduction electrons. It is seen from equations (V.33) and 
(V.34) that $(-\mathrm{J} / \mathrm{N}) \lambda_{1}\left(E_{k}, \epsilon_{k^{\prime}}\right)$ and $(-\mathrm{J} / \mathrm{N}) \cdot \lambda_{2}\left(E_{\kappa}, \epsilon_{k^{\prime}}\right)$ have dimen 3 ions of energy. Thus it seems natural to interpret them as exchange correlation energies, for in our discussion in the previous section we had seen explicitly that we had to deal with both, direct and exchange terms, with the additional requirement of Pauli Exclusion principle for the intermediate state sums. Furthermore, it is obvious that $\lambda_{1}\left(\varepsilon_{k}, \epsilon_{k^{\prime}}\right)$ and $\lambda_{2}\left(E_{k}, \epsilon_{k^{\prime}}\right)$ are in general complex functions. Hence, another way to interpret the introduction of $\lambda_{1}$ and $\lambda_{2}$ would be to consider the scattering of electrons from a complex effective potential. With this interpretation of $\lambda_{1}$ and $\lambda_{2}$ we find that we are close to Doniach's ${ }^{(20)}$ introduction of effective potentials in summing up his infinite class of diagrams. However, Doniach has summed up only leading order diagrams in an attempt to evaluate quantitles analogous to our $\lambda_{1}$ and $\lambda_{2}$ and we shall later on show that his results are the second order expansion of an approximate solution of the equation we shall obtain for these exchange correlation energies in the next section.

The physical implication of the complex nature of $\lambda_{1}\left(\varepsilon_{k} \epsilon_{k^{\prime}}\right)$ and $\lambda_{2}\left(\epsilon_{k}, \epsilon_{k^{\prime}}\right)$ is simply reflecting what we already knew intuitively, namely that in the scattering of an electron off an isolated impurity atom, the generation of particle + hole excitations leads to inelastic contributions to the cross section. As such, we find that the effect of the interaction between the s-electrons of the conduction band and the localized d-electrons of the magnetic impurity is to change the energy of the single electron levels and to give these states a certain width at the same time. This width is related to the imaginary 
part of the now complex energy while the energy shift is the real part. The broadening of the single particle energy levels is equivalent to giving a life time to the single particle electronic levels. Thus we find that our particles are now better looked upon as "quasi-particles" in the presence of the interaction. However, we would like to use the term "quasi-particle" with a little caution. From the plethora of work done on interacting many fermion systems we now know that the term "quasi-particle" in the conventional sense has a well defined meaning only near the fermi energy. Anticipating this to be so for our interacting fermion system too, we should like to reserve the interpretation of our electronic states as quasi-particles, only near the fermi energy.

For the present we limit our remarks on the functions $\lambda_{1}$ and $\lambda_{2}$, and proceed with the solution of equations (V.33) and (V. 34). During the analysis we shall have occasions to consider further the physical and theoretical implications of these functions which are perhaps best discussed side by side with the analysis.

Transferring the terms containing $\left\langle\phi_{N}, k^{\prime} \eta ; S_{\tau} / \psi_{k}\right\rangle$ and $\left\langle\phi_{N}, k^{\prime} l_{j}\left(\xi_{z}+1\right) / \psi_{k}\right\rangle$ to the left hand side of equations (V. 33) and (V.34) respectively and dividing the equations by the resulting coefficients of the se amplitudes, we obtain in a straight forward manner,

$$
\left\langle\phi_{N_{1}} k^{\prime} \uparrow_{j} S_{z} \mid \psi_{k}\right\rangle=\frac{i \eta \delta_{K K^{\prime}}}{E_{k}-\left(\varepsilon_{0}+\epsilon_{K^{\prime}}\right)-(-J / N)\left\{M \cdot \Delta u+\lambda_{1}\left(E, \epsilon_{k^{\prime}}\right)\right\}+i \eta}+
$$


$-85-$

$$
\begin{aligned}
& (-J / N)\left\{E_{k}-\left(\varepsilon_{0}+\epsilon_{k^{\prime}}\right)-(-J / N)\left\{M \cdot \Delta n+\lambda_{1}\left(E_{k}, \epsilon_{k^{\prime}}\right)\right\}+i \eta\right\}^{-1} \\
& {\left[M \sum_{k^{\prime \prime}}\left\langle\phi_{N^{\prime}}, k^{\prime \prime} \uparrow, s_{z} \mid \psi_{k}\right\rangle\right.} \\
& \left.+\sqrt{(s-M)(s+M+1)} \sum_{k^{\prime \prime}}\left\langle\phi_{N^{\prime}} k^{n} \downarrow \downarrow_{j}\left(s_{z}+1\right) \mid \psi_{k}\right\rangle\right]
\end{aligned}
$$

and

$$
\begin{aligned}
& \left\langle\phi_{N}, k^{\prime} \downarrow ;\left(s_{z}+1\right) \mid \psi_{k}\right\rangle \\
& =(-J / N)\left\{E_{K}-\left(\varepsilon_{0}+\epsilon_{K^{\prime}}\right)-(-J / N)\left\{(M+1) \Delta n+\lambda_{2}\left(E_{K^{\prime}}, \epsilon_{K^{\prime}}\right)\right\}+i \eta\right\}^{-1} \\
& {\left[-(M+1) \sum_{k^{\prime \prime}}\left\langle\phi_{N_{1}} k^{\prime \prime} \downarrow ;\left(S_{z}+1\right) \mid \psi_{k}\right\rangle\right.} \\
& \left.\left.+\sqrt{(s-M)(s+M+1) \cdot \sum_{k^{\prime \prime}}}\left\langle\phi_{N}, k^{n} \uparrow ; s_{z}\right| \psi_{k}\right)\right]
\end{aligned}
$$

A look at the energy denominators occurring in equations (V.35) and (V.36) immediately tells us that the effect of the inclusion of higher particle intermediate states is essentially to modify the energy levels of the system. In the language of Greens function or 
propagators, we have seen here the modification of unperturbed propagator, $\quad\left\{E_{k}-\left(\varepsilon_{0}+\epsilon_{k^{\prime}}\right)^{2}+i \eta\right\}^{-1}$ to the full propagators

$$
\left[E_{k}-\left(\varepsilon_{0}+\epsilon_{k^{\prime}}\right)-(-\nabla / N)\left\{M \cdot \Delta n+\lambda_{1}\left(E_{k}, \epsilon_{k^{\prime}}\right)\right\}+i \eta\right]^{-1}
$$

and

$$
\left[E_{k}-\left(\varepsilon_{0}+\epsilon_{k^{\prime}}\right)-(-J / N)\left\{(M+1) \Delta \eta+\lambda_{2}\left(E_{k}, \epsilon_{k^{\prime}}\right)\right\}+i \eta\right]^{-1}
$$

characterizing the propagation of the particle in the spin non-flipped and spin-flipped states respectively. In more sophisticated terms, we find that the presence of the interaction amongst all the electrons, brought about via the exchange interaction of each electron with the magnetic impurity (which is another way of simply stating the effect of higher particle intermediate states) calls for a "renormalization", (to use the standard terminology of field theory) of the energies.

Let us write, for brevity, $\left.\left(E_{k}-\varepsilon_{0}\right)\right)=\omega_{k},(-J / N)\left\{M \Delta a n+\lambda_{1}\left(E_{k}, \epsilon_{K^{\prime}}\right)\right\}$ $=\Lambda_{1}\left(E_{K^{\prime}}, \epsilon_{k^{\prime}}\right)$ and $(-J / N)\left\{(M+1) \cdot \Delta n+\lambda_{2}\left(E_{k}, E_{k^{\prime}}\right)\right\}=\Lambda_{2}\left(E_{K^{\prime}}, \epsilon_{k^{\prime}}\right)$. Then our full propagators become $\left\{\omega_{k}-\epsilon_{k^{\prime}}-n_{1}\left(\omega_{k}, \epsilon_{k^{\prime}}\right)\right\}^{-1}$ and $\left\{\omega_{k^{\prime}}-\epsilon_{k^{\prime}}-n_{2}\left(\omega_{k^{\prime}}, \epsilon_{k^{\prime}}\right)\right\}^{-1}$.

It is clear by now that summing equations (V.35) and (V.36) over $\mathrm{k}^{\prime}$ we obtain two algebraic equations for the closed sums $\sum_{k, 1}\left\langle\phi_{N}, k^{\prime \prime} \uparrow_{j} s_{z} / \psi_{k}\right\rangle$ and $\sum_{k, \prime}\left\langle\phi_{N}, k^{\prime \prime} \downarrow_{j}\left(s_{z}+1\right) \mid \psi_{k}\right\rangle$, needed to know the spin non-flip and spin-flip, single particle t-matrices. This step is identical with the one shown in section (V.A), where we solved the scattering equations within the single particle intermediate states, except for one crucial difference. There the propagators involved were the unperturbed propagators while now we have the full propagators in our scattering equations. 
$-87-$

Solving the simultaneous algebraic equations obtained from (V. 35) and (V. 36) we obtain in a straight forward fashion, the following,

$$
\begin{aligned}
A= & \sum_{k^{\prime \prime}}\left\langle\phi_{N}, k^{\prime \prime} \eta_{j} s_{z} \mid \psi_{k}\right\rangle \\
= & {\left[1-(-J / N) M x_{1}-(-J / N)^{2}(s-M)(s+M+1)\right.} \\
& \left.\cdot x_{i} x_{2}\left\{1+(-J / N)(M+1) x_{2}\right\}^{-1}\right]^{-1} \\
B= & \sum_{k=1}\left\langle\phi_{N}, k^{\prime \prime} \downarrow ;\left(s_{z}+1\right) / \psi_{k}\right) \\
= & (-J / N) \sqrt{(S-M)(s+M+1)} x_{2} \\
& \cdot\left[1+(-J / N)(M+1) x_{2}\right]^{-1} \cdot A
\end{aligned}
$$

where,

$$
\begin{aligned}
& \chi_{1}\left(\omega_{k}\right)=\sum_{k^{\prime}}\left\{\omega_{k}-\epsilon_{k^{\prime}}-\Lambda_{1}\left(\omega_{k}, \epsilon_{k^{\prime}}\right)+i \eta\right\}^{-1} \\
& \chi_{2}\left(\omega_{k}\right)=\sum_{k^{\prime}}\left\{\omega_{k}-\epsilon_{k^{\prime}}-\Lambda_{2}\left(\omega_{k}, \epsilon_{k^{\prime}}\right)+i \eta\right\}^{-1}
\end{aligned}
$$


$-88-$

Let us define the quantities $s(-J / N)[M \cdot A+\sqrt{(S-M)(S+M+1)} \cdot B]$ and $(-J / N)[-(M+1) \cdot B+\sqrt{(S-M)(S+M+1)} \cdot A] \quad$ occurring in equations (V.35) and (V.36) as $T_{\text {non-flip }}$ and $T_{\text {flip }}$ respectively. Then using (V. 37) and (V.38), we have after a few straight forward simplificatrons,

$$
\begin{aligned}
T_{n f}= & (-J / N)\left\{M+(-J / N) s(s+1) X_{2}\left(\omega_{k}\right)\right\} \\
& {\left[1-(-J / N)\left\{M X_{1}\left(\omega_{k}\right)-(M+1) X_{2}(\omega k)\right\}\right.} \\
& \left.-(-J / N)^{2} s(s+1) X_{1}\left(\omega_{k}\right) \cdot X_{2}\left(\omega_{k}\right)\right]^{-1}
\end{aligned}
$$

$$
\begin{aligned}
T_{f}= & (-J / N) \sqrt{(S-M)(s+M+1)} \\
& \cdot\left[1-(-J / N)\left\{M X_{1}\left(\omega_{k}\right)-(M+1) X_{2}\left(\omega_{k}\right)\right\}\right. \\
& \left.-(-J / N)^{2} s(S+1) X_{1}\left(\omega_{k}\right) X_{2}\left(\omega_{k}\right)\right]^{-1}
\end{aligned}
$$

(V. 40)

Equations (V. 35) and (V. 36) now become 


$$
\begin{aligned}
\left\langle\phi_{N}, k^{\prime} \uparrow ;\right. & s_{z}\left|\psi_{k}\right\rangle \\
= & \left\{i \eta \delta_{k k^{\prime}}+T_{n_{f}}\right\} \\
& \left\{\omega_{k}-\epsilon_{k^{\prime}}-\Lambda_{1}\left(\omega_{k}, \epsilon_{k^{\prime}}\right)+i \eta\right\}^{-1}
\end{aligned}
$$

$$
\begin{aligned}
& \left\langle\phi_{N_{1}, k^{\prime} \downarrow}\left(s_{z}+1\right) \mid \psi_{k}\right\rangle \\
& =T_{f} \cdot\left\{\omega_{k}-\epsilon_{k^{\prime}}-\Lambda_{2}\left(\omega_{k}, \epsilon_{k^{\prime}}\right)+i \eta\right\}^{-1}
\end{aligned}
$$

Equations (V.41) and (V.42), along with the expressions (V. 39) and (V. 40) constitute the "Formal" solution for the spin non-flip and spin-flip scattering amplitudes. We have called the solution "Formal" for it still remains to determine the functions $\Lambda_{1}\left(\omega_{k}, \epsilon_{k^{\prime}}\right)$ and $\Lambda_{2}\left(\omega_{k}, \epsilon_{k^{\prime}}\right)$. We proceed to do this in the next section. It seems proper to menti on though, that as we would expect, $\Lambda_{1}\left(\omega_{*}, G_{*}\right)$ and $\Lambda_{2}\left(\omega_{k}, \epsilon_{k}\right)$ are not independent of each other. 
D. Self Consistent Equation for Self Energy

It is well known now that the self-energy, usually denoted by $\sum(\omega, k)$ in many body theory, is given by the forward scattering amplitude. This standard result of field theory, has been made use of by many author s in considering the present problem by various methods. However, almost always, the attempt invariably has been to sum up infinite classes of Feynman diagrams to evaluate it. Using such a procedure Brenig and GUtze ${ }^{(18)}$ have provided an expression for self energy of the present problem in terms of the spin non-flip scattering amplitude. It is to be noted though that their expression does not include all the diagrams corresponding even to three particle scattering states, let alone any higher contributions. We could make use of the standard result mentioned above to obtain an expression for our self-energy in terms of the spin nonflip scattering amplitude, for it shall immediately give us a relation between $\Lambda_{1}\left(\omega_{k}, \epsilon_{k^{\prime}}\right)$ and $T_{n f}\left(k, k^{\prime}\right)$, which itself involves $1_{1}\left(\omega_{k}, \varepsilon_{k}\right)$ through the function $x_{1}\left(\omega_{k}\right)$. Hence we shall have an integral equation to determine $\Lambda_{1}\left(w_{x}, \epsilon_{x}\right)$ in a selfconsistent way. However, instead of making use of this standard result, we shall derive the same from first principles by invoking the boundary conditions to be satisfied by the quantities we defined as t-matrices in section (IV. C). This shall give us a greater insight into concepts of formal field theoretic scattering as applied to therm odynamic systems. 
$-91$.

For details of what is involved here, we refer to a beautiful paper by Gell-Mann and Goldberger!(38) For the purposes of discussion here, let us recall the basic definition of our spin non-flip matrix, (equation V.33),

$$
t_{n f}\left(k, k^{\prime}\right)=T_{n f}\left(k, k^{\prime}\right)+\Lambda_{1}\left(w_{k}, \epsilon_{k^{\prime}}\right)\left\langle\phi_{n,} k^{\prime} \uparrow ; S_{z} \mid \psi_{k}\right\rangle
$$

where we have made use of definition of $\mathrm{T}_{\mathrm{nf}}\left(\mathrm{K}, \mathrm{K}^{\prime}\right)$. Using equation (V.41), we finally obtain,

$$
\begin{aligned}
t_{n f}\left(k, k^{\prime}\right)= & {\left[T_{n f}\left(k, k^{\prime}\right)+\Lambda_{1}\left(\omega_{k}, \epsilon_{k^{\prime}}\right)\right.} \\
& \left\{\left\{i \eta \delta_{k k^{\prime}}+T_{n f}\left(k, k^{\prime}\right)\right] .\right. \\
\cdot & {\left.\left.\left[\omega_{k}-\epsilon_{k^{\prime}}-\Lambda_{1}\left(\omega_{k}, \epsilon_{k^{\prime}}\right)+i \eta\right]^{-1}\right\}\right] }
\end{aligned}
$$

Simplifying we have,

$$
\begin{aligned}
& t_{n_{f}}\left(k, k^{\prime}\right)= T_{n f}\left(k, k^{\prime}\right)\left\{1+\frac{\Lambda_{1}\left(\omega_{k}, \epsilon_{k^{\prime}}\right)}{\omega_{k}-\epsilon_{k^{\prime}}-\Lambda_{1}\left(\omega_{k}, \epsilon_{k^{\prime}}\right)+i \eta}\right\} \\
&+i \eta \delta_{k k^{\prime}} \Lambda_{1}\left(\omega_{k}, \epsilon_{k^{\prime}}\right) \\
& \cdot\left\{\omega_{k}-\epsilon_{k^{\prime}}-\Lambda_{1}\left(\omega_{k}, \epsilon_{k^{\prime}}\right)+i \eta\right\}^{-1}
\end{aligned}
$$


The second term on the right involves a Kronecker delta, which we recall arises from the orthonormalized character of the eigen functions of the unperturbed hamiltonian. We know that in the limit of an infinite normalizing volume, $\mathrm{V} \rightarrow \infty$, the $\mathrm{Kr}$ onecker delta is replaced by a Dirac delta function. Hence taking the the rmodynamic limit $\mathrm{N} \rightarrow \infty, \mathrm{V} \rightarrow \infty$ such that $(\mathrm{N} / \mathrm{V})$ remains a constant, our Kronecker delta shall get replaced by a Dirac delta function, $\delta\left(K-K^{\prime}\right)$, which for the one value of $K=K^{\prime}$ tends to blow up. However, from formal field theoretic scattering theory we know that as $\mathrm{V} \rightarrow \infty$ and $\eta \rightarrow \mathrm{o}^{+}, \mathrm{t}_{\mathrm{nf}}\left(\mathrm{K}, \mathrm{K}^{\prime}\right)$ must tend to zero, since otherwise the cross section would be infinite, (for reasons just explained) or at least dependent upon the normalization volume. Hence for an interaction that produces self-energy effects which are not infinitesimal, the term $\Lambda_{1}\left(\omega_{k}, \epsilon_{k^{\prime}}\right) \cdot \delta_{\kappa k^{\prime}}$ must be cancelled by a portion of the other term on the right hand side of (V.43). Thus the expression (V.43), must be of the form of an infinitesimal plus a term proportional to $\delta_{\mathrm{KK}^{\prime}}$, that does not vanish as $\quad \eta \longrightarrow 0^{+}$and $\mathrm{V} \rightarrow \infty$. Direct application of this results to (V. 43) for $K=K^{\prime}$ immediately gives us,

$$
\Lambda_{1}\left(w_{k}, \epsilon_{k}\right)=-T_{n f}(k, k)
$$

where $\omega_{k}$ and $\epsilon_{k}$ are related by the equality, $\omega_{k}=\epsilon_{k}+\Lambda_{1}\left(\omega_{k}, \epsilon_{k}\right)$ Making use of our expression (V. 39) for $T_{n f}\left(K, K^{\prime}\right)$ we obtain the 
following equation to determine $\Lambda_{1}\left(\omega_{k}, \epsilon_{k}\right)$ self-consistently;

$$
\begin{aligned}
\Lambda_{1}\left(\omega_{k}, \epsilon_{k}\right)= & -(-J / N)\left\{M+(-J / N) s(s+1) X_{2}\left(\omega_{k}\right)\right\} \\
& \cdot\left[1-(-J / N)\left\{M X_{1}\left(\omega_{k}\right)-(M+1) X_{2}\left(\omega_{k}\right)\right\}\right. \\
& \left.-(-J / N)^{2} s(s+1) X_{1}\left(\omega_{k}\right) \cdot X_{2}\left(\omega_{k}\right)\right]^{-1}
\end{aligned}
$$

This is an integral equation for $\Lambda_{1}\left(\omega_{k}, \epsilon_{k}\right)$ since it involves $\Lambda_{1}\left(\omega_{k}, \epsilon_{k^{\prime}}\right)$ in the integral $x_{1}\left(\omega_{k}\right)$ through its definition,

$$
X_{1}\left(\omega_{k}\right)=\sum_{k^{\prime}}\left\{\omega_{k}-\Lambda_{1}\left(\omega_{k}, \epsilon_{k^{\prime}}\right)-\epsilon_{k^{\prime}}+i \eta\right\}^{-1}
$$

\section{E. Some Properties of the Functions Involved}

In the foregoing sections we obtained a formal solution for the spin non-flip and spin-flip scattering amplitudes in terms of the self energy. We then provided a self-consistent equation to determine the self-energy. It now remains to solve this equation for the self-energy. However, to do this we shall have to invoke certain mathematical properties of the functions involved. We, therefore, shall devote this section to such an investigation.

Let us first investigate the properties of the function $X_{1}\left(\omega_{k}\right)$ 
-94-

given by,

$$
X_{1}\left(w_{k}\right)=\sum_{k^{\prime}}\left\{\omega_{k}-\epsilon_{k^{\prime}}-n_{1}\left(\omega_{k}, \epsilon_{k^{\prime}}\right)+i \eta\right\}^{-1}
$$

In the thermodynamic limit of volume going to infinity, such that the number of electrons per unit volume remains constant, the summation over $k^{\prime}$ goes over to an integral, characterisetic of continuous spectrum. Thus, using the standard procedure of replacing a discrete sum by an integral, we have,

$$
\gamma_{1}\left(\omega_{k}\right)=\int_{-\infty}^{\infty} \frac{\rho\left(\epsilon_{k^{\prime}}\right)\left\{1-2 f\left(\epsilon_{k^{\prime}}-\epsilon_{f}\right)\right\}}{\omega_{k}-\epsilon_{k^{\prime}}-\Lambda_{1}\left(\omega_{k}, \epsilon_{k^{\prime}}\right)+i \eta} d \epsilon_{k^{\prime}}
$$

where $\rho\left(\epsilon_{k^{\prime}}\right)$ is the single particle density of states and $f\left(\epsilon_{k^{\prime}}-\epsilon_{p}\right)$ is the Fermi distribution function, $\epsilon_{F}$ being the chemical potential.

More explicitly we must write $X_{1}\left(\omega_{k}\right)$ as $X_{1}\left(\omega_{k}, T\right)$ since it depends on temperature through its dependence on the fermi function. Thus,

$$
X_{1}\left(\omega_{k}, T\right)=\int_{-\infty}^{\infty} \frac{\rho\left(\epsilon_{k^{\prime}}\right) \tanh \left\{\beta\left(\epsilon_{k^{\prime}}-\xi_{k}\right) / 2\right\}}{\omega_{k}-\epsilon_{k^{\prime}}-1_{1}\left(\omega_{k}, \sigma_{k^{\prime}}\right)+i \eta} d \epsilon_{k^{\prime}}
$$


where $\beta=\left(k_{B} T\right)^{-1}, k_{B}$ being Boltzman's constant. Changing the origin to the chemical potential, we have,

$$
X_{1}\left(\omega_{k}, T\right)=\int_{-\infty}^{\infty} \frac{\rho\left(\epsilon_{k^{\prime}}+\epsilon_{\beta}\right) \tanh \left\{\beta \epsilon_{k^{\prime} / 2}\right\}}{\omega_{k}-\epsilon_{k^{\prime}}-n_{1}\left(\omega_{k}, \epsilon_{k^{\prime}}\right)+i \eta} d \epsilon_{k^{\prime}}
$$

with the definition $\omega_{k}=E-\left(\varepsilon_{0}+\epsilon_{F}\right)$. Thus $\omega_{k}$ characterizes the single particle energies of the interacting system as opposed to $\epsilon_{k}$ which corresponds to the unperturbed single particle levels. Although the integral in (V.46) runs from $-\infty$ to $\infty$, we may make a finite cut off based on the physical limitations on the energies in the conduction band. We may for instance limit the integral from $-D$ to $D$, where $2 D$ is the conduction band width, by defining the density of single particle states to be nonvanishing only in this energy range. We shall consider the explicit form of the choice of our density of states later on, but may remark here that there is no loss of generality in the mathematical properties of $\mathcal{X}$, by restricting our selves to the energy range $-D$ to $D$, in which case we have,

$$
X_{1}\left(\omega_{k}, T\right)=\int_{-D}^{D} \frac{\rho\left(\epsilon_{k^{\prime}}+\epsilon_{1}\right) \tanh \left(\beta \epsilon_{k^{\prime}} / 2\right)}{\omega_{k}-\epsilon_{k^{\prime}}-n_{1}\left(\omega_{k_{1}} \epsilon_{k^{\prime}}\right)+i \eta} d \epsilon_{x^{\prime}}
$$


Let us consider $x_{1}$ as a function of complex energy $z$. We define,

$$
X_{1}(z, T)=\int \frac{\rho\left(z^{\prime}\right) \tanh \left(\beta z^{\prime} / 2\right)}{z-z^{\prime}-\Lambda_{1}\left(z, z^{\prime}\right)+i \eta} d z^{\prime}
$$

To consider the analytic properties of $X_{1}(Z, \beta)$, we have to first consider the basic density of states function $p(Z)$. We have already considered it to be non-vanishing only between $-D$ and $+D$. We further assume the following properties for $\rho(Z)$.

I).

$$
\int_{-D}^{D} \rho(\epsilon) d \epsilon=\text { finite }
$$

II). All singularities of $p(\mathrm{Z})$ in the complex plane are outside a large circle of radius $D$ ar ound the origin.

The first property is always satisfied for realistic band 8 . The second feature is characteristic of the conduction bands of metals. Since $\mathrm{D}$ corresponds to a temperature of the order of $10^{4 \circ} \mathrm{K}$ to $10^{50} \mathrm{~K}$, the condition $\mathrm{K}_{\mathrm{B}} \mathrm{T}<<\mathrm{D}$ is always satisfied for all temperatures of any possible interest. Thus it follows that $P(Z)$ is a smoothly varying function for all $Z<<D$, such that 
an appreciable change from the value $\rho(0)$ at the origin occurs for energies of the order of $D$ only.

According to Van Hove's theorem ${ }^{(39)}, \rho(\omega)$ has a square root singularity at the band edges. The simplest function having the se properties is

$$
\rho(\omega)=\rho(0) \cdot\left\{1-(\omega / D)^{2}\right\}^{1 / 2}
$$

The function $\rho(\omega)$ can be considered as the boundary value of the function,

$$
\rho(z)=\rho(0)\left\{1-(z / D)^{2}\right\}^{1 / 2}
$$

at the real axis. $\rho(Z)$ has two branch points at $\pm D$ and the branch cut is chosen along the real axis between $-D$ and $D$. Except for this branch cut, $\rho(Z)$ is holomorphic on the whole complex plane behaving at infinity like

$$
\rho(z) \sim-i \rho(0) \cdot(z / D)+\theta\{(D / z)\}
$$

For $E^{2} \leq D^{2}$ one finds that, 


$$
\rho(\epsilon \pm i \eta)= \pm \rho(\epsilon)
$$

Such a density of states has been considered by Brenig and Gbtze (18) Treatments for more generalized density of states function have been given by Mueller-Hartmann and Zittarz. (13) However, we should like to point out that the often used square well or Lorentzian density states, ${ }^{(15)}$ both of which violate Van Hove's theorem, cannot be considered as boundary values of holomorphic function obeying (V.47) at the cut.

With the se properties of $\rho(Z)$, it follows that the function $X_{1}(z, T)$ is holormorphic everywhere, excluding the branch cut from $-D$ to $+D$. It decreases at infinity like $Z^{-1}$. Furthermore, it is easily seen that the reflection principle, $X_{1}^{*}(z, T)=X_{1}\left(z^{*}, T\right)$ where * mean complex conjugate, is satiofied, which immediately gives us,

$$
\chi^{*}(\omega+i \eta, T)=\chi(\omega-i \eta, T)
$$

thus providing us with the discontinuity of $X_{1}$ across the branch cut as, 
-99-

$$
\begin{aligned}
\left\{X_{1}(\omega+i \eta, T)\right. & \left.-X_{1}(\omega-i \eta, T)\right\} \\
= & -2 \pi i \operatorname{Im} X_{1}(\omega, T)
\end{aligned}
$$

which is purely imaginary.

In an exactly analogous fashion we also obtain that $\mathcal{X}_{2}(Z, T)$ is analytic everywhere except from $-D$ to $+D$ on the real axis, where it has a branch cut. Also $\chi_{2}^{*}(Z, T)=\chi_{2}\left(z^{*}, T\right)$

Making use of the se properties of $X_{1}(z, T)$ and $X_{2}(z, T)$ along with the equation (V. 45) for $\Lambda_{1}(Z, \beta)$, we immediately conclude that $\Lambda_{1}(Z, \beta)$ being a combination of the sectionally holomorphic ${ }^{(40)}$ functions $X_{1}(Z, \beta)$ and $X_{2}(Z, \beta)$, must itself be at least sectionally holomorphic. Thus it follows that $\Lambda_{1}(Z, \beta)$ is analytic everywhere, with the possible exception of the real axis from $-D$ to $+D$. The reflection principle for $X_{1}$ and $X_{2}$ provides us with the information that,

$$
\begin{aligned}
& T_{n f}^{*}(z, \beta)=T_{n f}\left(z^{*}, \beta\right) \\
& T_{f}^{*}(z, \beta)=T_{f}\left(z^{*}, \beta\right)
\end{aligned}
$$

which also implies that,

$$
\Lambda_{1}^{*}(z, \beta)=\Lambda_{1}\left(z^{*}, \beta\right)
$$


and

$$
\Lambda_{2}^{*}(z, \beta)=\Lambda_{2}\left(z^{*}, \beta\right)
$$

It is perhaps worthwhile to point out that the functions $X_{1}$ and $\chi_{2}$ are nothing but the sums over the full single particle propagators. The analytic properties we have just proved above therefore involved nothing more than the analytic properties of single particle propagator $\mathrm{s}$ which have been investigated quite intensively by many body theorists interested in the Green's function approach to many particle systems. We have made use of some of such concepts as proved by J. M. Luttinger ${ }^{(41)}$ for the single particle propagator $\mathbf{s}$ in many fermion systems.

We now investigate limiting behavior of our functions. We see that functions $X_{1}(Z, \beta)$ and $X_{2}(Z, \beta)$ go to zero as $z^{-1}$ in the limit $\mathrm{Z} \rightarrow \infty$. This is in keeping with the well known behavior of single particle propagators. With this result applied to our expressions $(V .39)$ and $(V .40)$ for $T_{n f}$ and $T_{f}$ we immediately obtain,

$$
\operatorname{Lim}_{z \rightarrow \infty} T_{n f}(z, \beta)=(-J / N) M
$$

and

$$
\lim _{z \rightarrow \infty} T_{f}(z, \beta)=(-J / N) \sqrt{(S-M)(S+M+1)}
$$

The se are nothing but the first Born approximation terms for spin non-flip and spin flip t-matrices. For the case of $s$ pin $S=\frac{1}{2}$ 
impurity, we find that for scattering from $S_{Z}=M=\frac{1}{2}$ state the non-flip t-matrix becomes $\quad(1 / 2)(-J / N)$ in the Born approximation, while the spin-flipt-matrix is zero. This is in agreement with the limiting behavior for $t-m$ atrices as obtained from Suhl's dispersion theoretic formulation of the problem. By virtue of (V. 44) and (V.49), it also follows that

$$
\lim _{z \rightarrow \infty} \Lambda_{1}(z, \beta)=-(-J / N) M
$$

Thus we see that the exchange-correlation energy, $\Lambda_{1}(z, \beta)$ instead of going to zero for large energies, goes to a real constant $(J / N) \cdot M$. Recalling the result of our solution written the single particle intermediate states in section (V.A) we find that $(\mathrm{J} / \mathrm{N}) \mathrm{M}$ was associated with the molecular field renormalization of the chemical potential and of the magnetic moments of the conduction electrons. 


\section{EVALUATION OF SELF ENERGY}

\section{A. Approximate Evaluation and Comparison With Previous $\underline{\text { Results }}$}

To know the self energy exactly, we have to solve equation (V.45) exactly. The aim of our theoretical work is to be able to improve the existing results on the self energy in the hope that its unphysical behavior can be removed by a better treatment of the problem. This shall also allow us to consider the nature of the ground state. Furthermore, to obtain physical parameters a knowledge of the self-energy is necessary as well as sufficient because determination of the self-energy for small values of the complex energy $Z$, yields directly the relevant non-equilibrium parameters like electrical conductivity, thermo-electric power, magnetoresistence etc. Also, the thermodynamic properties of the system are given by the asymptotic behavior of the electron self-energy for large $\mathrm{Z}$. Thus, ideally one would like to know the nature of the self-energy without any approximations. We wo uld be successful in realizing our aim provided we can solve the integral equation (V. 45) for our self-energy without any approximations. fortunately, we notice that if the electron self-energy $\Lambda_{1}\left(\omega_{k}, \epsilon_{k^{\prime}}, \beta\right)$ occurring in the definition of the full propagators through,

$$
G_{k k^{\prime}}=\left\{\omega_{k}-\epsilon_{k^{\prime}}-\Lambda_{1}\left(\omega_{k}, \epsilon_{k^{\prime}}, \beta\right)+i \eta\right\}^{-1}
$$


is considered to be independent of $\epsilon_{K^{\prime}}$ in the integration range $-D$ to $D$ for the function $X_{l}\left(\omega_{k}, \beta\right)$, then the integral equation (V. 45) reduces to a transcendental equation. We have been able to solve this transcendental equation. Although it is not very satisfying to obtain the self-energy under this approximation, we may take some consolation in the fact that our results under this approximation are already a considerable improvement over existing results. In particular we only mention here (and shall show later on) that the quantity analogous to our $\Lambda_{1}$ which Doniach ${ }^{(20)}$ in his formulation has called effective potential, turns out to be the second order perturbation expansion in power 8 of $\mathrm{J}$, of the first order iterative solution of the integral equation (V. 45) for $\Lambda_{1}(Z, \beta)$.

Before we proceed, a word of caution on our approximation is necessary. It is well known that the poles of the propagators provide the single particle excitation energies. Thus for the exact propagator we see that poles occur whenever the relation,

$$
\omega_{k}=\left\{\epsilon_{k_{r}}+\Lambda_{1}\left(\omega_{k}, \epsilon_{k_{r}}, \beta\right)\right\}
$$

is satisfied, $\epsilon_{K_{r}}$ being the root of this equation. Since the function $\Lambda_{1}\left(\omega_{k}, \epsilon_{x^{\prime}}, \beta\right)$ is unknown at this point, we cannot aprion say how many roots, $\epsilon_{K_{\gamma}}$. we may have. Restricting $\Lambda_{1}$ to be a function of $\omega_{K}$ alone thus imposes the condition that there is one and only 
$-104$

one root given by,

$$
\epsilon_{k_{r}}=\left\{\omega_{k}-\Lambda\left(\omega_{k}, \beta\right)\right\}
$$

Physically this amounts to an apriori restriction that one and only one unperturbed single particle state $\epsilon_{\mathrm{K}_{r}}$ goes into the exact state of energy $\omega_{K^{*}}$. In this sense then, treating $\Lambda_{1}$ as a function of $\omega_{K}$ alone restricts the behavior of our system to "Normal" systems. (42) If more than one root $\epsilon_{\mathrm{K}_{r}}$ is a possibility, physically it would imply a collective phenomenon of some sort in which many unperturbed single particle states go into a final state of energy $\omega_{\mathrm{K}^{*}}$. Thus it is well to keep in mind that ar approximaltion may be leading us away from such a phenomenon.

Under the approximation $\Lambda_{1}\left(\omega_{k}, \epsilon_{k^{\prime}}\right)=\Lambda_{1}\left(\omega_{k}, \epsilon_{k}\right)$ we obtain from (V. 45) the following transcendental equation,

$$
\begin{aligned}
\Lambda_{1}\left(\omega_{k}, \beta\right) & =-(-J / N)\left\{M+(-J / N) s(s+1) X_{2}\left(\omega_{k}-\Lambda_{2}\left(\omega_{k}, \beta\right)\right)\right\} \\
& \cdot\left[1-(-J / N)\left\{M X_{1}\left(\omega_{k}-\Lambda_{1}\left(\omega_{k}, \beta\right)\right)-(M+1) X_{2}\left(\omega_{k}-\Lambda_{2}\left(\omega_{k}, \beta\right)\right)\right\}\right. \\
& \left.-(-J / N)^{2} s(s+1) X_{1}\left(\omega_{k}-\Lambda_{1}\left(\omega_{k}, \beta\right)\right) \cdot X_{2}\left(\omega_{k}-\Lambda_{2}\left(\omega_{k}, \beta\right)\right)\right]^{-1}
\end{aligned}
$$

(VI. 1) 
$-105-$

where $X_{1}$ and $X_{2}$ are given by,

$$
X_{1}\left\{\omega_{k}-\Lambda_{1}\left(w_{k}, \beta\right)\right\}=\int_{-\infty}^{D} \frac{\rho_{0}\left(\epsilon_{k^{\prime}}+\epsilon_{F}\right) \tanh \left(\beta \epsilon_{k^{\prime}} / 2\right)}{\omega_{k}-\epsilon_{k^{\prime}}-\Lambda_{1}\left(\omega_{k}, \beta\right)+i \eta} d \epsilon_{k^{\prime}}
$$

and

$$
X_{2}\left\{\omega_{k}-\Lambda_{2}\left(\omega_{k}, \beta\right)\right\}=\int_{-0}^{J} \frac{\rho_{0}\left(\epsilon_{k^{\prime}}+\epsilon_{F}\right) \tanh \left(\beta \epsilon_{k^{\prime} / 2}\right)}{\omega_{k}-\epsilon_{k^{\prime}}-\Lambda_{2}\left(\omega_{k}, \beta\right)+i \eta} d \epsilon_{k^{\prime}}
$$

In the se expressions we have written $\Lambda_{1}$ and $\Lambda_{2}$ only as a function of $\omega_{K}$ for because of the energy conservation rebaions $\omega_{k}=\epsilon_{k}+\Lambda_{1}\left(\omega_{k}, \beta\right)$ and $\omega_{k}=\epsilon_{k}+\Lambda_{2}\left(\omega_{k}, \beta\right)$, we immediately see that it is redundant to write them as functions of $\omega_{K}$ and $\epsilon_{K}$ both. It also follows from the se relations that under our approximation of treating $\Lambda_{1}$ and $\Lambda_{2}$ to be indpendent of $\epsilon_{K^{\prime}}, \Lambda_{1}\left(\omega_{k}, \beta\right)=\Lambda_{2}\left(\omega_{k}, \beta\right)$. Thus we find that $X_{1}=X_{2}=X$ say, and equation $(\mathrm{Vl} \cdot 1)$ reduces to

$$
\begin{aligned}
\Lambda\left(\omega_{k}, \beta\right)= & -(-J / N)\left\{M+(-J / N) s(s+1) X\left(\omega_{k}-\Lambda\left(\omega_{k}, \beta\right)\right)\right\} \\
\cdot & {\left[1+(-J / N) X\left(\omega_{k}-\Lambda\left(\omega_{k}, \beta\right)\right)-(-J / N)^{2}\right.} \\
& \left.\cdot s(s+1) X^{2}\left(\omega_{k}-\Lambda\left(\omega_{k}, \beta\right)\right)\right]^{-1}
\end{aligned}
$$


where $\mathcal{X}$ is given by,

$$
\begin{aligned}
X\left\{\omega_{k}-\Lambda\left(\omega_{k}, \beta\right)\right\} & =\int_{-0}^{p} \frac{\rho_{0}\left(\epsilon_{k^{\prime}}+\epsilon_{k}\right) \tanh \left(\beta \epsilon_{k^{\prime}} / 2\right)}{\omega_{k}-\epsilon_{k^{\prime}}-\Lambda\left(\omega_{k}, \beta\right)+i \eta} d \epsilon_{k^{\prime}} \\
& =X(\bar{\omega}, \beta)
\end{aligned}
$$

At this stage we should like to put our results in a form which would ease camparison with previously obtained results. We find that this is best achieved by transforming our results to the total angular momentum representation. Defining the total angular momentum as $j$, we have $j=S \pm \frac{1}{2}$.

Thus using Clebsch-Gordon coefficients we find that for the total angular momentum channel $j=\left(S+\frac{1}{2}\right)$ we have,

$$
\begin{aligned}
\Lambda_{j=s+1 / 2}(\omega, \beta)= & -(-J / N)\{s+(-J / N) s(s+1) x\} \\
& \cdot\left[1+(-J / N) x-(-J / N)^{2} s(s+1) x^{2}\right]^{-1}
\end{aligned}
$$

and for total angular momentum channel $j=S-\frac{1}{2}$, 


$$
\begin{aligned}
\Lambda_{j=s-1 / 2}(\omega, \beta)= & -(-J / N)\{-(s+1)+(-J / N) s(s+1) x\} \\
& {\left[1+(-J / N) X-(-J / N)^{2} s(s+1) X^{2}\right]^{-1} }
\end{aligned}
$$

(VI. 5)

where we have made use of the rotational invariance of the Hamiltonian. In the total angular momentum channel a further simplification of the expressions for $\Lambda_{j=S+\frac{1}{2}}$ and $\Lambda_{j}=S-\frac{1}{2}$ results. The denominators of (VI. 4) and (VI.5) are the same and can be factorized as,

$$
\{1-(-7 / N) s x\} \cdot\{1+(-7 / N)(S+1) X\}
$$

We notice that in equations (VI.4) and (VI.5) one of the factors in the denominator is the same as the numerator and hence cancels out, providing us with the following results,

$$
\bigwedge_{j=s+1 / 2}(\omega, \beta)=-(-J / N) s[1-(-J / N) s X(\omega, \beta)]^{-1}
$$

and 


$$
\Lambda_{j=s-1 / 2}(\omega, \beta)=(-J / N)[1+(-J / N)(s+1) \times(\bar{\omega}, \beta)]^{-1}(S+1)
$$

(VI. 7)

Before we get involved in a discussion of the mumerical solution of the transcendental equations (VI.6) and (VI. 7), let us consider the first iterative solution for $\Lambda$ s by setting $\Lambda(\omega, \beta)=0$ in the definition of the integral $X(\bar{\omega}, \beta)$ (equation VI. 3). We do this, for we find that the other various results for the energy difference between the unperturbed and the perturbed ground states happen to be approximations of our first iterative solution for $\Lambda$. Thus a comparison of our first order iterative solution (for which we can provide explicit expressions without resorting to numerical analysis) with the existing results not only checks our results in limiting cases but also shows convincingly that the numerical results obtained from the full transcendental equations are a considerable improvement over the se existing results.

Thus, setting $\Lambda(\omega, \beta)=0$ in the definition of the integral $X(\tilde{\omega}, \beta)$ we obtain the following equations for the first order iterative solution for $\Lambda$ 's,

$$
\Lambda_{j=s+1 / 2}(\omega, \beta)=(J / N) \sigma[1+(J / N) s \chi(\omega, \beta)]^{-1}
$$


and

$$
\Lambda_{j=s-1 / 2}(\omega, \beta)=(-J / N)(s+1)[1-(v / N)(s+1) X(\omega, \beta)]^{-1}
$$

where

$$
X(\omega, \beta)=\int_{-\infty}^{D} \frac{f_{0}\left(t_{x^{\prime}}+\epsilon_{p}\right) \tanh \left(\beta \epsilon_{k^{\prime} / 2}\right)}{\omega-\epsilon_{k^{\prime}}+i \eta} d \epsilon_{k^{\prime}}
$$

(VI. 10)

At this point we should like to compare our results with Doniach's. (20) Expanding the expression (VI.8) and (VI.9) in powers of $\mathrm{J}$, we obtain, up to the second order in $\mathrm{J}$,

$$
\Lambda_{j=s+1 / 2}(\omega, \beta)=(J / N) s[1-(J / N) s X(\omega, \beta)]
$$

$$
\bigwedge_{j=s-1 / 2}(\omega, \beta)=-(J / N)(s+1)[1+(J / N)(s+1) X(\omega, \beta)]
$$

These are the same as the results obtained by Doniach for his $\mathrm{V}_{\text {eff }}^{\mathrm{j}=\mathrm{S} \pm \frac{1}{2}}$ (equation 33, Ref. 20), with one minor difference in 
the constants. Our second order terms have the coefficients $\mathrm{S}^{2}$ and $(\mathrm{S}+1)^{2}$ while his terms have $\mathrm{S}$ and $(\mathrm{S}+1)$.

To obtain explicit expressions for $\Lambda_{j=S \pm 1 / 2}^{(1)}(\omega, \beta) \quad$ we have to evaluate the all important integral $X(\omega, \beta)$ (equation VI-10). Because of the fact that the experimentally observed physical parameters do not show any marked dependence on the band structure, there shall be no loss of qualitative information on the nature of solution in taking the density of states $\rho_{0}\left(\epsilon_{k^{+}}^{\prime}+\epsilon_{F}\right)$ to be equal to $\rho_{0}\left(\epsilon_{F}\right)$, the density of states at the fermi energy, in the range $-D$ to $+D$. Also, we have already pointed out in the previous section that the density of states function is different from $\rho_{0}\left(\epsilon_{F}\right)$ only for energies of the order of $D$. Treating the density of states as a slowly varying function of energy, we replace $\rho_{0}\left(\epsilon_{k^{\prime}}+\epsilon_{F}\right)$ by $\rho_{0}\left(\epsilon_{F}\right)$ and are thus able to evaluate the integral (VI.10) quite simply.

We evaluate (VI. 10) in appendix 2 , under the condition $k_{B} T<<D$, which is satisfied for all temperatures of interest. The result is,

$$
\begin{aligned}
X(\omega, \beta)=-2 \rho_{0}\left(\epsilon_{F}\right)\left[\frac{1}{2} \cdot \ln \left\{\frac{D^{2}-\omega^{2}}{(2 \pi i / \beta)^{2}}\right\}\right. \\
\left.-\psi\left(\frac{1}{2}+\frac{\omega}{(2 \pi 2 / \beta)}\right)\right]
\end{aligned}
$$


where $\psi(z)$ is the di-gamma function. ${ }^{(43)}$ Combining the expression (VI. 13) for $X(\omega, \beta)$ with equations (VI. 8 ) and (VI. 9) we have now an explicit expression for the first order iterative solutions for $\Lambda_{j=S \pm \frac{1}{2}}^{(1)}(\omega, \beta)$. We now investigate the behavior of the se solutions in the asymptotic limits of the di-gamma function. The asymptotic form of the di-gamma function $\psi(z)$ for $z \rightarrow \infty$ in $\operatorname{larg} \mathrm{Z} \mid<\pi \quad$ is given by, (ref. 43, equation 6.3.18).

$$
\psi(z)-\ln z-\frac{1}{2 z}-\frac{1}{12 z^{2}}+\frac{1}{120 z^{4}}-\cdots \cdot
$$

Thus for the low temperature limit $\left(\omega / k_{B} T\right)>>1$, we have

$$
\begin{aligned}
\psi\left(\frac{1}{2}+\frac{\omega}{2 \pi i k_{B} T}\right) \sim & \ln \left(\frac{1}{2}+\frac{\omega}{2 \pi i k_{B} T}\right)-\frac{1}{2}\left(\frac{1}{2}+\frac{\omega}{2 \pi i k_{B} T}\right)^{-1} \\
& +\Theta\left\{\left(\frac{1}{2}+\frac{\omega}{2 \pi i k_{B} T}\right)^{-1}\right\}^{2}
\end{aligned}
$$

From the expression (VI. 13) for $\chi$ we have,

$$
\begin{aligned}
X(\omega, \beta)= & -2 \rho_{0}\left(\epsilon_{F}\right)\left[(1 / 2) \ln \left\{\frac{D^{2}-\omega^{2}}{\left(2 \pi i k_{B} T\right)^{2}}\right\}\right. \\
& \left.-\ln \left\{\frac{1}{2}+\frac{\omega}{2 \pi i k_{B} T}\right\}+\left\{1+\frac{\omega}{2 \pi i k_{B} T}\right\}^{-1}\right]
\end{aligned}
$$


Where we have retained terms only to the order $z^{-1}$ in $\psi(z)$. Rearranging term 8 , we have,

$$
\begin{aligned}
X(\omega, \beta) \approx- & 2 \rho_{0}\left(\epsilon_{F}\right)\left[(1 / 2) \ln \left\{\frac{\omega^{2}-D^{2}}{\left(\omega+\pi i k_{B} T\right)^{2}}\right\}\right. \\
& \left.+\left\{1+\frac{\omega}{\pi i k_{B} T}\right\}^{-1}\right]
\end{aligned}
$$

(VI. 14)

We may further simplify this expression to obtain,

$$
\begin{aligned}
X(\omega, \beta)= & -2 \rho_{0}\left(\epsilon_{F}\right)\left[\left(y_{2}\right) \ln \left\{\frac{D^{2}-\omega^{2}}{\omega^{2}+\left(\pi k_{B} T\right)^{2}}\right\}\right. \\
& +\frac{\pi^{2} k_{B}^{2} T^{2}}{\omega^{2}+\left(\pi k_{B} T\right)^{2}}+i\left\{\frac{\omega \pi k_{B} T}{\omega^{2}+\left(\pi k_{B} T\right)^{2}}\right. \\
& \left.\left.-\tan ^{-1}\left(\frac{\pi k_{B} T}{\omega}\right)\right\}\right]
\end{aligned}
$$

In the limit of $T \rightarrow$ o, we have finally,

$$
X(\omega, T \rightarrow 0) \approx \frac{1}{2} \ln \left(\frac{D^{2}-\omega^{2}}{\omega^{2}}\right)
$$

From (VI.8), (VI.9) and (VI. 15), we obtain the following expressions for $A^{\prime} s$ at absolute zero, 


$$
\begin{array}{r}
\Lambda_{j=S+1 / 2}(\omega)=(J / N) S\left[1-\rho_{0}\left(\epsilon_{F}\right)(J / N) S\right. \\
\left.\cdot \ln \left(\frac{D^{2}-\omega^{2}}{\omega^{2}}\right)\right]^{-1}
\end{array}
$$

(VI. 16)

$$
\begin{array}{r}
\Lambda_{j=s-1 / 2}(\omega)=-(J / N)(s+1)\left[1+\rho_{0}\left(\epsilon_{F}\right)(J / N)(s+1)\right. \\
\left.\cdot \ln \left(\frac{D^{2}-\omega^{2}}{\omega^{2}}\right)\right]^{-1}
\end{array}
$$

(VI. 17)

The se expressions for $\bigwedge_{j=S \pm \frac{1}{2}}$ at absolute zero develop poles at value $s$ of $W$ given by,

$$
1=\rho_{0}\left(\epsilon_{F}\right)(J / N) S \ln \left(\frac{D^{2}-\omega^{2}}{\omega^{2}}\right)
$$

(VI. 18)

and

$$
1=-\rho_{0}\left(\epsilon_{F}\right)(\tau / N)(s+1) \ln \left(\frac{D^{2}-\omega^{2}}{\omega^{2}}\right)
$$

From the se we obtain,

$$
\omega_{B}\left(j=s+y_{2}\right)= \pm D\left\{e^{y / r \mid s}+1\right\}^{-1 / 2}
$$




$$
\omega_{B}\left(j=s-y_{2}\right)= \pm D\left\{e^{1 /|r|(s+1)}+1\right\}^{-1 / 2}
$$

where $\quad \gamma$ is the dimensionless constant, $\rho_{0}\left(t_{F}\right) \cdot(J / N)$.

From the expressions (V. 70) and (V.71) it is seen that the $\boldsymbol{\omega}$ 's are non-analytic functions of the interaction strength $\mathrm{J}$.

Thus we find that the interacting ground state, for both the channels, $j=\left(S_{ \pm} \frac{1}{2}\right)$, developes a kind of "Binding Energy" corresponding to the negative sign in (VI. 20) and (VI. 21). This again reflects the fact that the present problem cannot be treated within the realm of perturbation theory. The binding energy corresponds to the reduction of the entropy of the conduction electrons. In expressions (VI. 20) and (VI.21), if the consider the limit $r$ very small, the "Weak Coupling" limit, we obtain the results obtained by other s; ${ }^{(5)}$

$$
\omega_{B}(j=s+1 / 2)= \pm D \exp \{-1 / 2|r| \cdot s\}
$$

$$
\omega_{B}(j=s-1 / 2)= \pm D \exp \{-1 / 2|v| \cdot(s+1)\}
$$


$-115$

Let us now consider the other asymptotic limit $k_{B} T \gg \omega$, subject to the condition $D \gg k_{B} T$ under which we have the expression for $X(\omega, T)$ as,

$$
\begin{aligned}
X(\omega, T) \approx-2 \rho_{0}\left(\epsilon_{F}\right)\left\{\left(y_{8}\right) \ln \right. & \frac{D^{2}-\omega^{2}}{\left(2 \pi k_{B} T\right)^{2}} \\
& \left.-\psi\left(\frac{1}{2}+\frac{\omega}{2 \pi k_{s} T}\right)\right\}
\end{aligned}
$$

Thus, in the high temperature limit $\left(\omega / k_{B} T\right)<<1$ we have,

$$
X(\omega, T) \approx-2 \rho_{0}\left(\epsilon_{F}\right)\left\{\frac{1}{2} \ln \frac{D^{2}}{\left(2 \pi k_{B} T\right)^{2}}-\psi(1 / 2)\right\}
$$

which combined with (VI. 8) and (VI. 9) gives us

$$
\Lambda_{j=s+1 / 2}=(J / N) s\left[1-2 \int_{0}\left(\epsilon_{F}\right)(J / N) s\left\{\ln \left(D / 2 \pi k_{B} T\right)-\psi\left(Y_{2}\right)\right\}\right]^{-1}
$$

(VI. 24)

$$
\begin{gathered}
\Lambda_{j=s-1 / 2}=-(J / N)(s+1)\left[1+2 \rho_{0}\left(\epsilon_{F}\right)(J / N)(s+1)\left\{\ln \left(D / 2 \pi k_{\beta} T\right)\right.\right. \\
-\psi(1 / 2)\}]^{-1}
\end{gathered}
$$


These expressions for the energy corrections in the two channels $\mathrm{j}=\mathrm{S} \pm \frac{1}{2}$ develop poles at temperatures,

$$
T_{6}\left(j=s+y_{2}\right)=\left(D / 2 \pi k_{B}\right) \exp \left\{-1 / 2|r| s-\psi\left(y_{2}\right)\right\}
$$

and,

$$
T_{c}\left(j=s-y_{2}\right)=\left(D / 2 \pi k_{B}\right) \exp \{-1 / 2|r|(s+1)-\psi(1 / 2)\}
$$

where $\psi\left(\frac{1}{2}\right)=-1.9635$ and $J \geqslant 0$ is under stood for $j=S \pm \frac{1}{2}$ respectively. Such poles have been obtained by the other approaches as well and the critical temperature for $\mathrm{J}<0$ which occurs for the antiferomagnetically coupled channel $j=\left(S-\frac{1}{2}\right)$ has become known as the Kondo temperature in the literature.

The occurrence of these critical temperatures however shows that the high temperature results (VI. 24) and (VI.25) are valid only for temperature regions much above the critical temperature. In the region around the critical temperatures for the two channels, we have to take the full form of di-gamma function in our expression for $X(\omega, T)$, equation (VI.13). If we were to combine the explicit expressions for energy corrections obtained for zero temperature and very high temperatures, and extrapolate them 
toward the critical temperatures, one would obtain the qualitative blowing up behavior of energy change as a function of temperature obtained within the limitations of other theoretical work. However, from our exact self-consistent transcendental equations (VI.6) and (VI. 7), one can immediately see that the energy corrections can never blow up, since the right hand side tends to reach a constant value as $\wedge \rightarrow \infty$, contradicting the left hand side and thus violating the self-consistent determining equations for $\Lambda$ 's themselves. This behavior clearly shows that the energy correction instead of blowing up at some critical temperature, and having an infinite discontinuity at that temperature, (results obtained by others up till now) shall show a maximum as a function of temperature with a smooth continuous behavior all through. A numerical evaluation of $\Lambda$ for both the channels $j=\left(S \pm \frac{1}{2}\right)$ performed by us shows this behavior and confirms our qualitative interpretation of our results. Based on the physical nature of the present problem and the experimental results available, such a behavior for $\mathrm{J}<0$ has often been speculated and conjectured by many. (5) We show here, for the first time, that the inclusion of the higher particle intermediate states (or exchang e-correlation effects) and its selfconsistent evaluation even within the approximation where $\wedge\left(\omega_{k}, C_{k^{\prime}}\right)$ is considered to be the same for all the unperturbed single particle states by putting $\Lambda\left(\omega_{k}, \epsilon_{k^{\prime}}\right)=\Lambda\left(\omega_{k} \epsilon_{k}\right)$ removes the blowing up character of the energy correction to the unperturbed ground state energy, as a function of temperature, thus providing meaningful result s. 
An additional new feature of our solution for the $s-d$ exchange model is related to the case $\mathrm{J}>0$. It is important to note that the ferromagnetically coupled case (i.e., J $>$ o) is believed to be free of any peculiar behavior and is assumed to be adequately and correctly treated within perturbation theory. We have shown that this belief is unfounded. The $s-d$ exchange model is capable of giving rise to ferromagnetically coupled resonant state and is consequently capable of describing impurity ferromagnetism.

B. Num erical Analysis

We present here some details of the numerical analysis of the transcendental equations for $\Lambda_{j=S \pm \frac{1}{2}}$. To evaluate the integral,

$$
X(\omega, \beta)=\int_{-D}^{D} \frac{\rho_{0}\left(\epsilon^{\prime}\right) \tanh \left(\beta \epsilon^{\prime} / 2\right)}{\omega-\epsilon^{\prime}-\Lambda(\omega, \beta)+i \eta} d \epsilon^{\prime}
$$

we separate it into its real and imaginary parts by using the Pmelj identities, (ref. 40, page 42) commonly known as,

$$
\frac{1}{x-x^{\prime} \pm i \eta}=\text { P.P. } \frac{1}{x-x^{\prime}} \mp i \eta \delta\left(x-x^{\prime}\right)
$$

As discussed in the previous section the physical parameters of 
the dilute magnetic alloys being rather insensitive to details of the band structure, we take the density of states to be essentially constant at its Fermi energy value in the conduction band. We chose a band of half width, $|\mathrm{D}|,=5 \mathrm{eV}$. Using Simpson's rule we have evaluated the principle part of $X(\omega, \beta)$ for temperatures in the range $1^{\circ} \mathrm{K}$ to $300^{\circ} \mathrm{K}$ and for energies in the range $-D$ to $D$.

Knowing $X(\omega, \beta)$ we solve numerically the transcendental equations for $\Lambda_{j}(\omega, \beta)$. The numerical analysis was done for impurity spin, $S=\frac{1}{2}$ and for various fixed values of the density of states at Fermi energy, $\rho_{0}\left(\epsilon_{F}\right)$.

In figs. (3) and (4) we have shown the real parts of the self-energies, corresponding to the antiferromagnetically and ferromagnetically coupled channels respectively, as a function of temperature, at the Fermi energy. In figs. (5) and (6) we plot the corresponding imaginary parts. The critical temperatures are taken to be the temperatures where the real parts become zero. These lead to resonances in the scattering amplitudes and hence justify their interpretation as temperatures where the resonant state starts building up.

Figs. (7) and (8) show the real and imaginary parts of $\wedge$ for the ferromagnetically coupled channel, evaluated for the same values of the parameter $\rho_{0}\left(\epsilon_{F}\right)$ and $|J|$ as for the antiferromagnetic case plotted in figs. (3) and (5). Note the absence of a critical temperature in fig. (6) down to $1^{\circ} \mathrm{K}$. We 
shall discuss this behavior in the section on interpretation of result s.

Figs. (9) and (10) show the behavior of the critical temperature as a function of the magnitude of interaction strength, for given values of $\rho_{0}\left(\epsilon_{F}\right)$. Figs. (11) and (12) give a plot of the critical temperatures as a function of $f_{0}\left(\epsilon_{\beta}\right)$ for given values of $|J|$. 


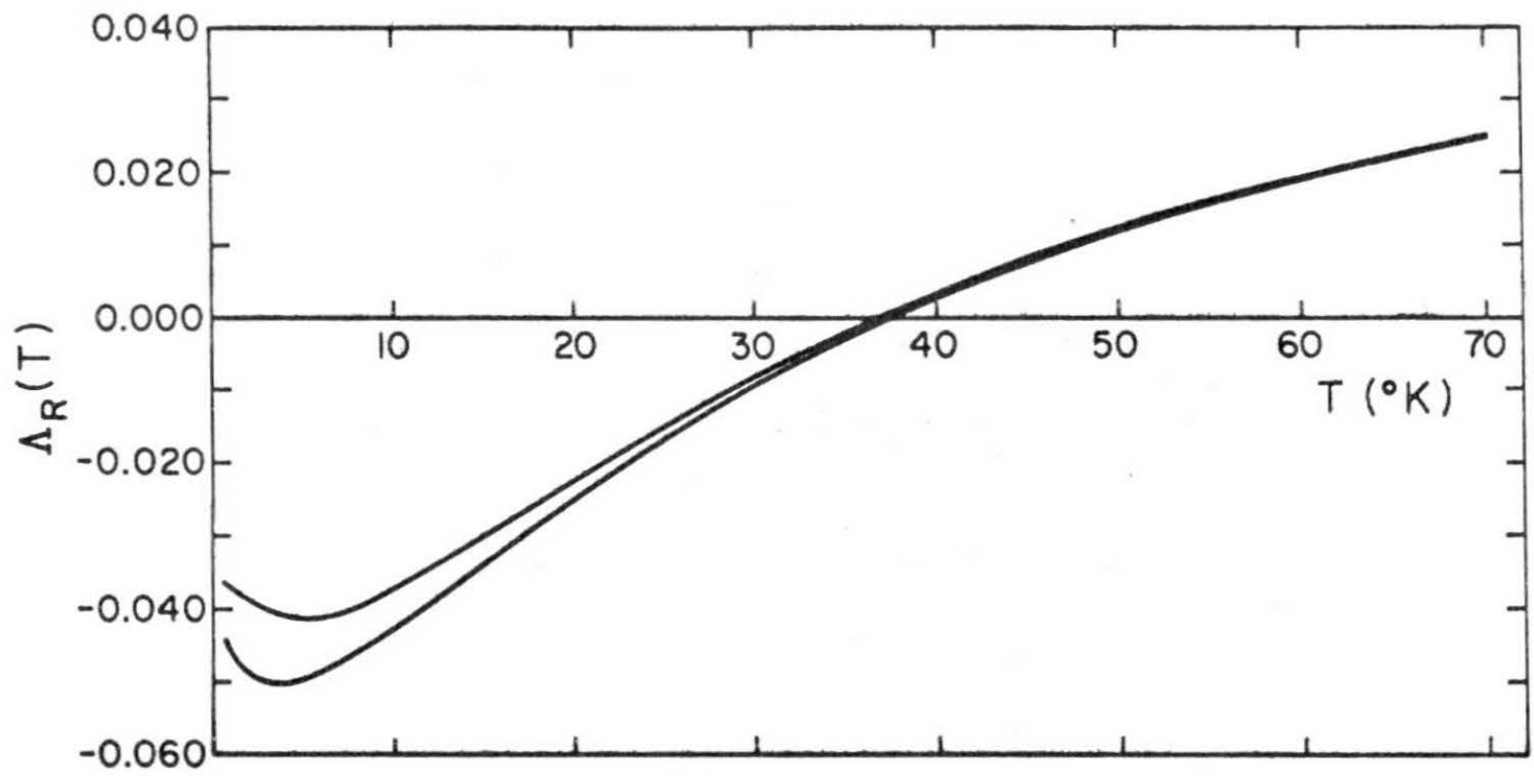

Fig. 3. Real part of the self-energy for anti-ferromagnetic The lower curve corresponds to Fermi energy while the upper curve is evaluated for $x=0.001 \mathrm{ev}, x$ being measured from Fermi energy. 


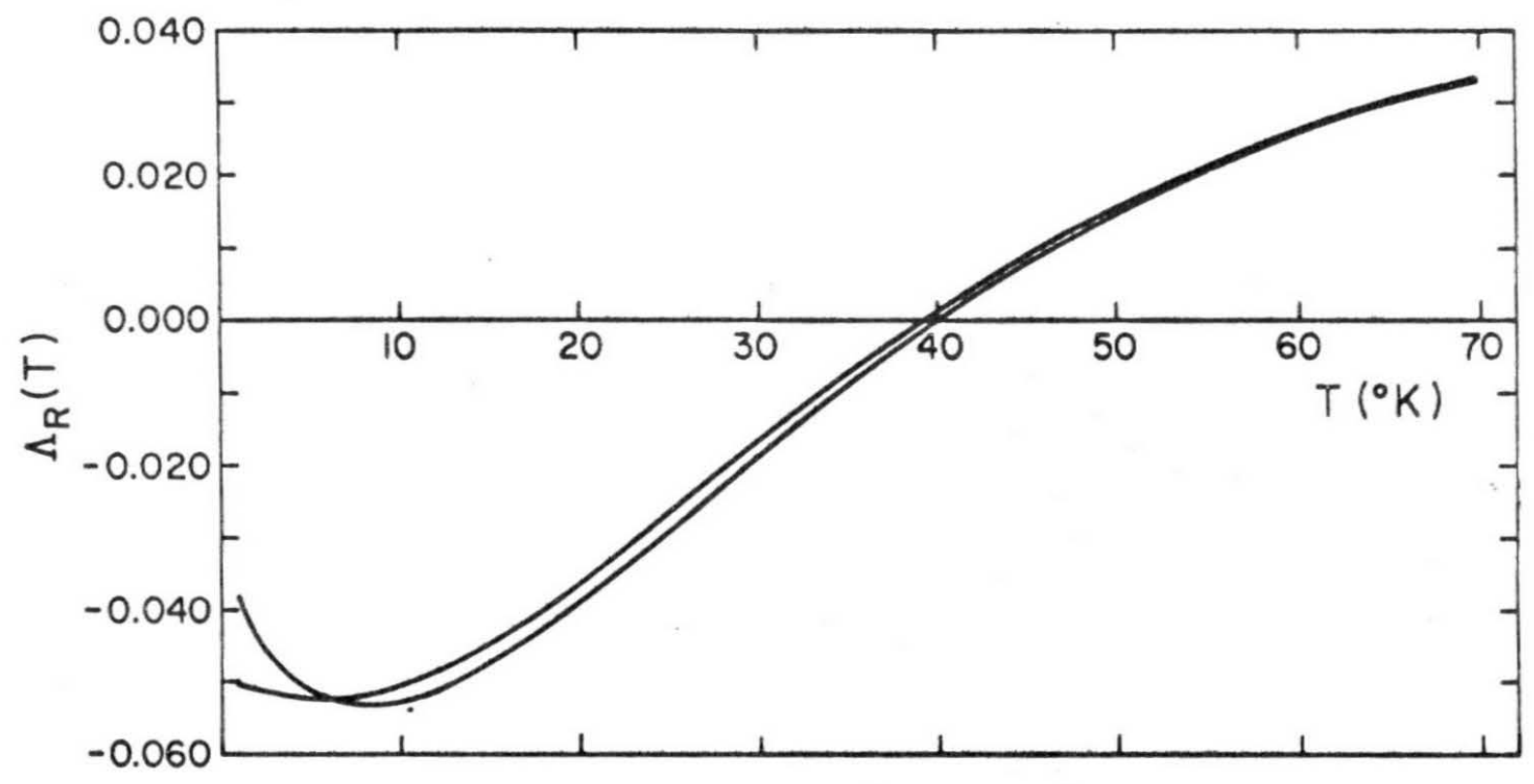

Fig. 4 Real part of self-energy for ferromagnetic coupling $J=0.045 \mathrm{ev}, p_{0}=(10 / 1.5 \pi)$ atom -1 ev. -1 lower curve is evaluated at fermi energy while the upper is for $x=0.001 \mathrm{ev}$ away from fermi energy. 


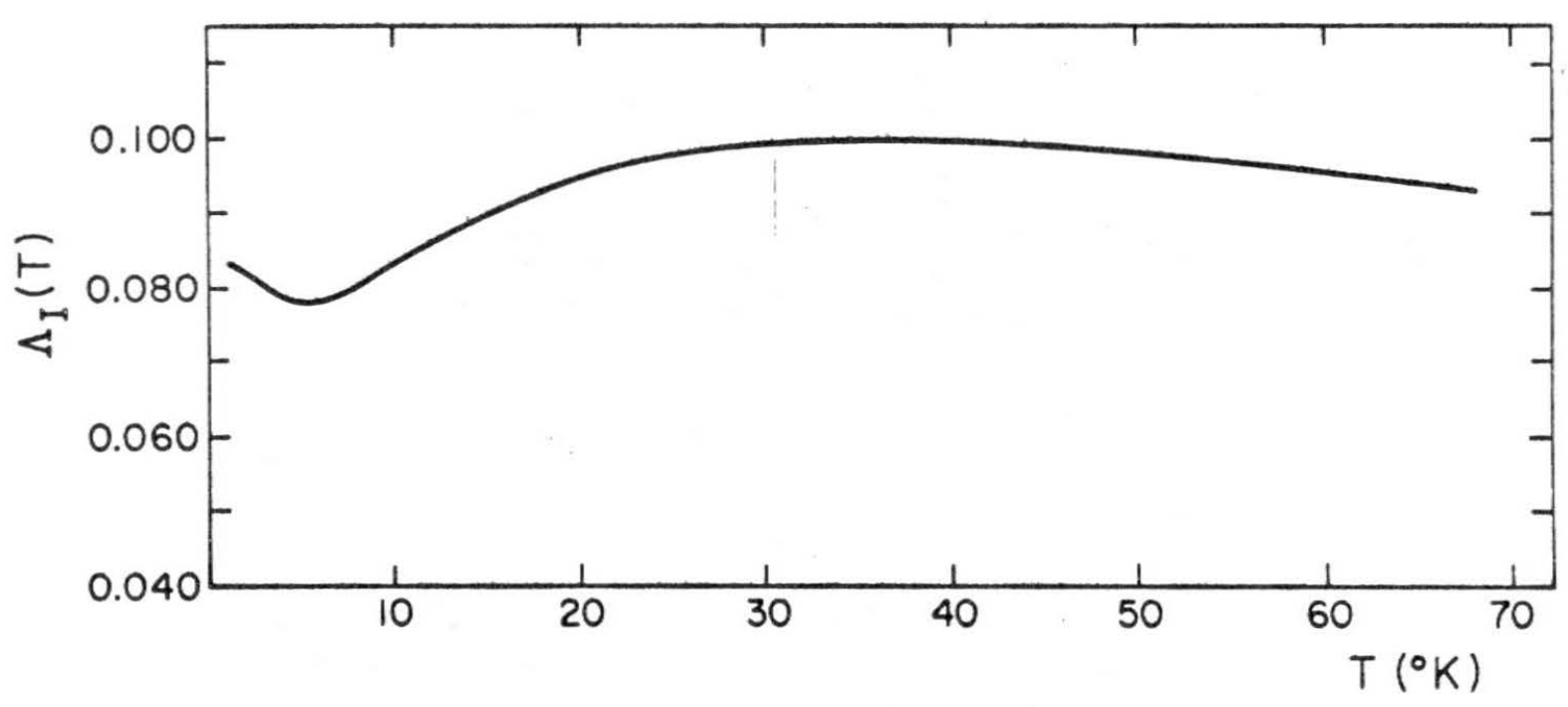

Fig. 5 Imaginary part of the self-energy for anti ferromagnetic coupling, $J=-0.021 \mathrm{ev}, P_{0}=$
evaluated at the fermi energy. 


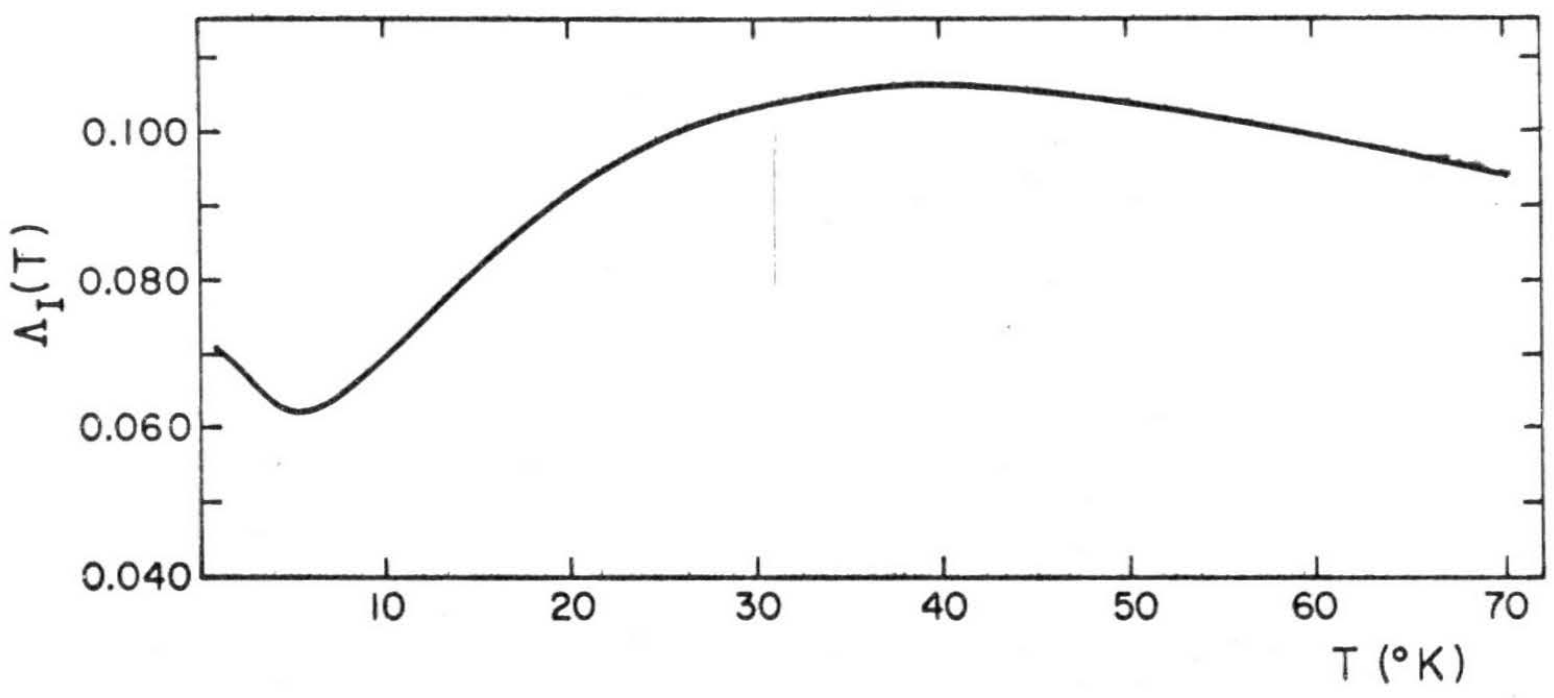

Fig. 6 Imaginary part of the self-energy for ferromagnetic coupling, $J=0.045 \mathrm{ev}, \rho_{0}=(10 / 1.5 \pi)$ atom $^{-1} \mathrm{ev}^{-1}$, evaluated at the fermi energy. 


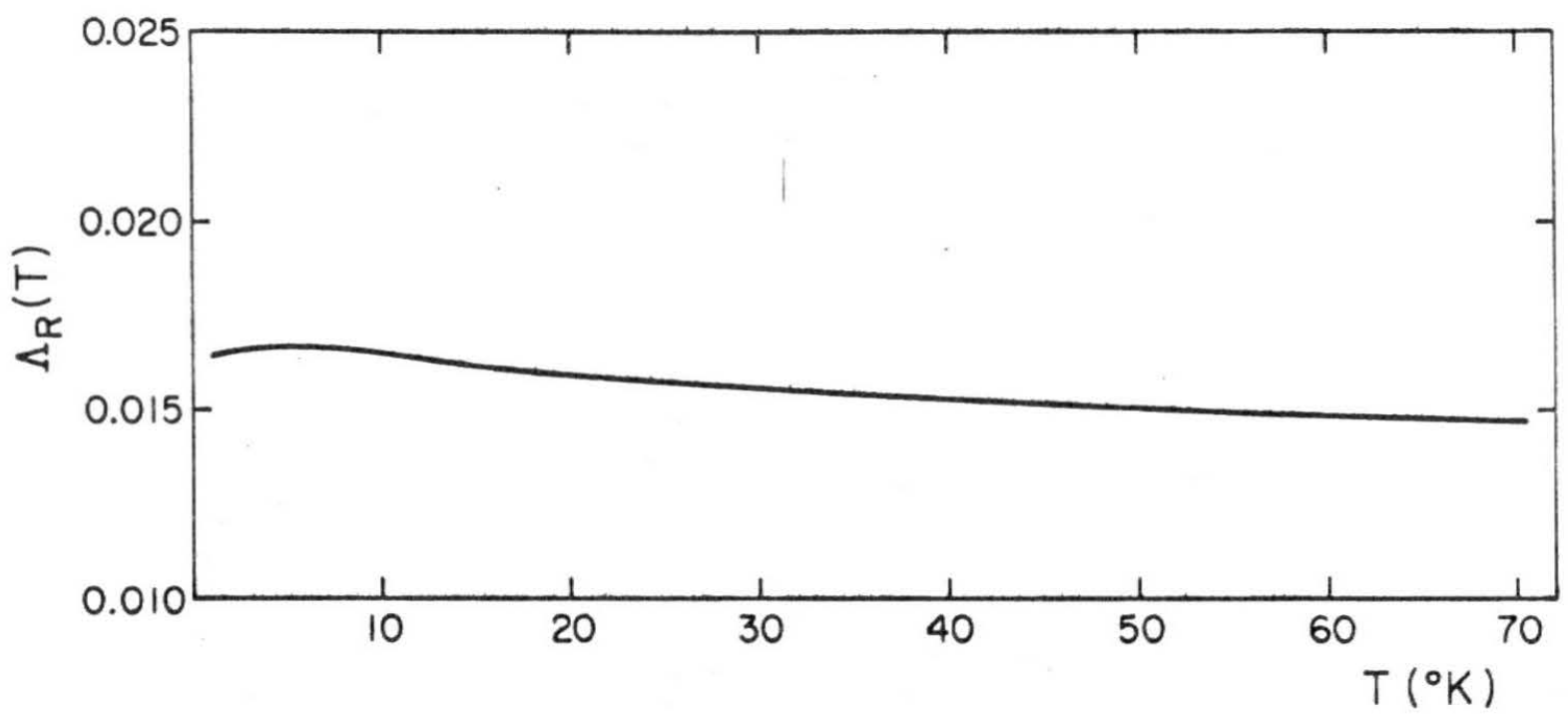

Fig. 7 Real part of self-energy for ferromagnetic coupling using the same magnitude of $\mathrm{J}=0.021 \mathrm{ev}$ as for antiferromag ne tic coupling shown in Fig. 3. Note the absence of a critical temperature down to $1^{\circ} \mathrm{K}$. 


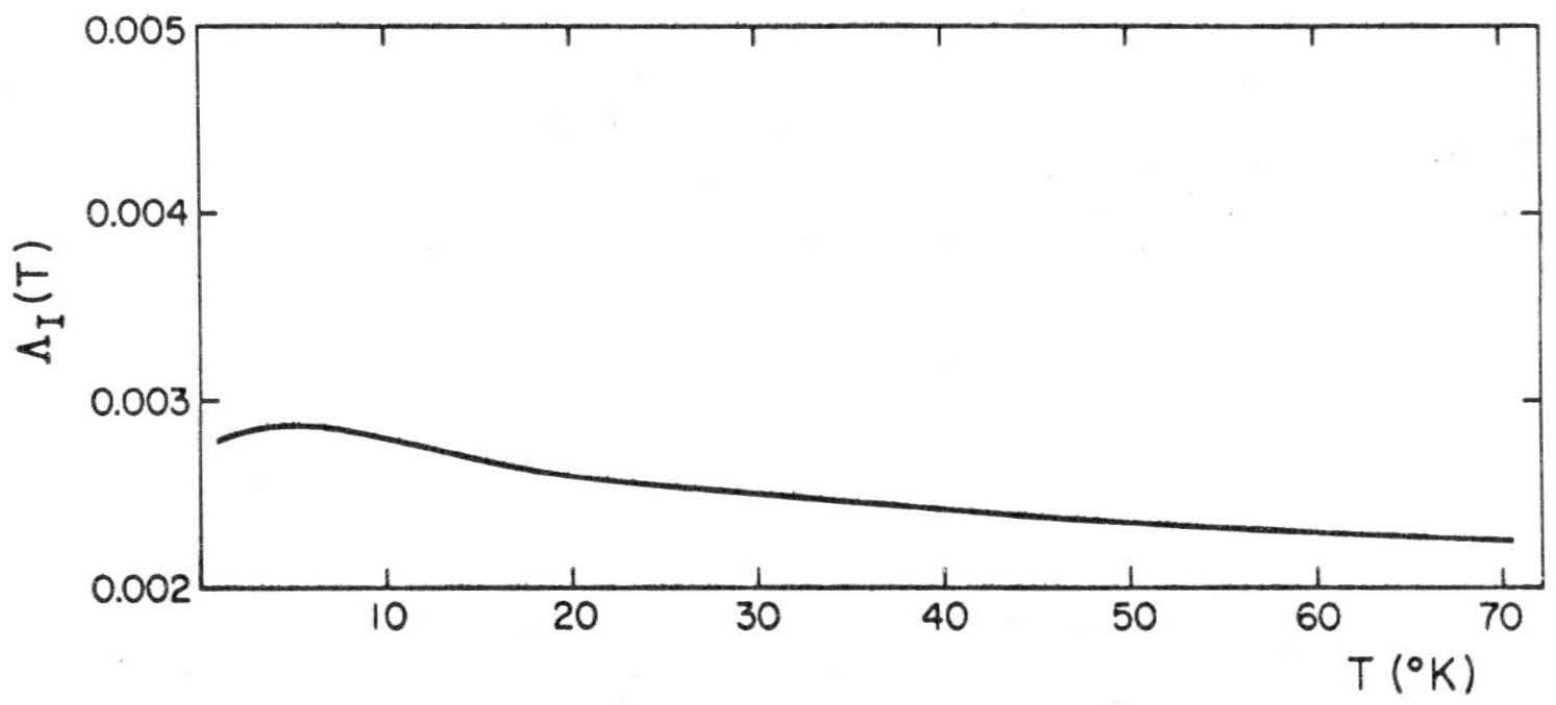

Fig. 8 Imaginary part of self-energy for ferrom agnetic coupling using the same magnitude of $J=0.021 \mathrm{ev}$ as for antifer romagnetic coupling shown in Fig. 5. 


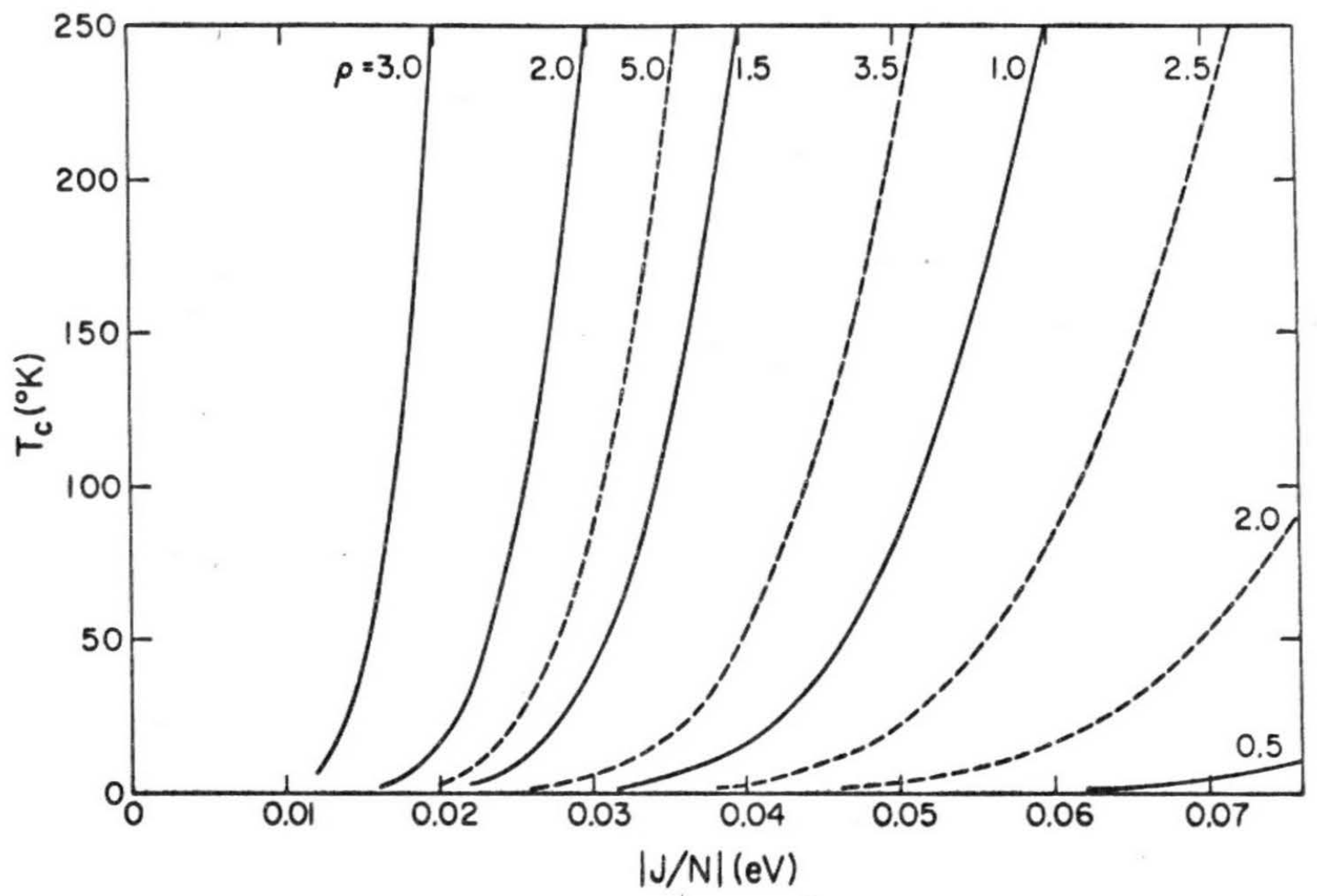

Fig. 9 Critical temperature plotted against exchange interaction strength. Solid curves correspond to $\mathrm{J}<0$ and dashed curves to $\mathrm{J}>0$. 


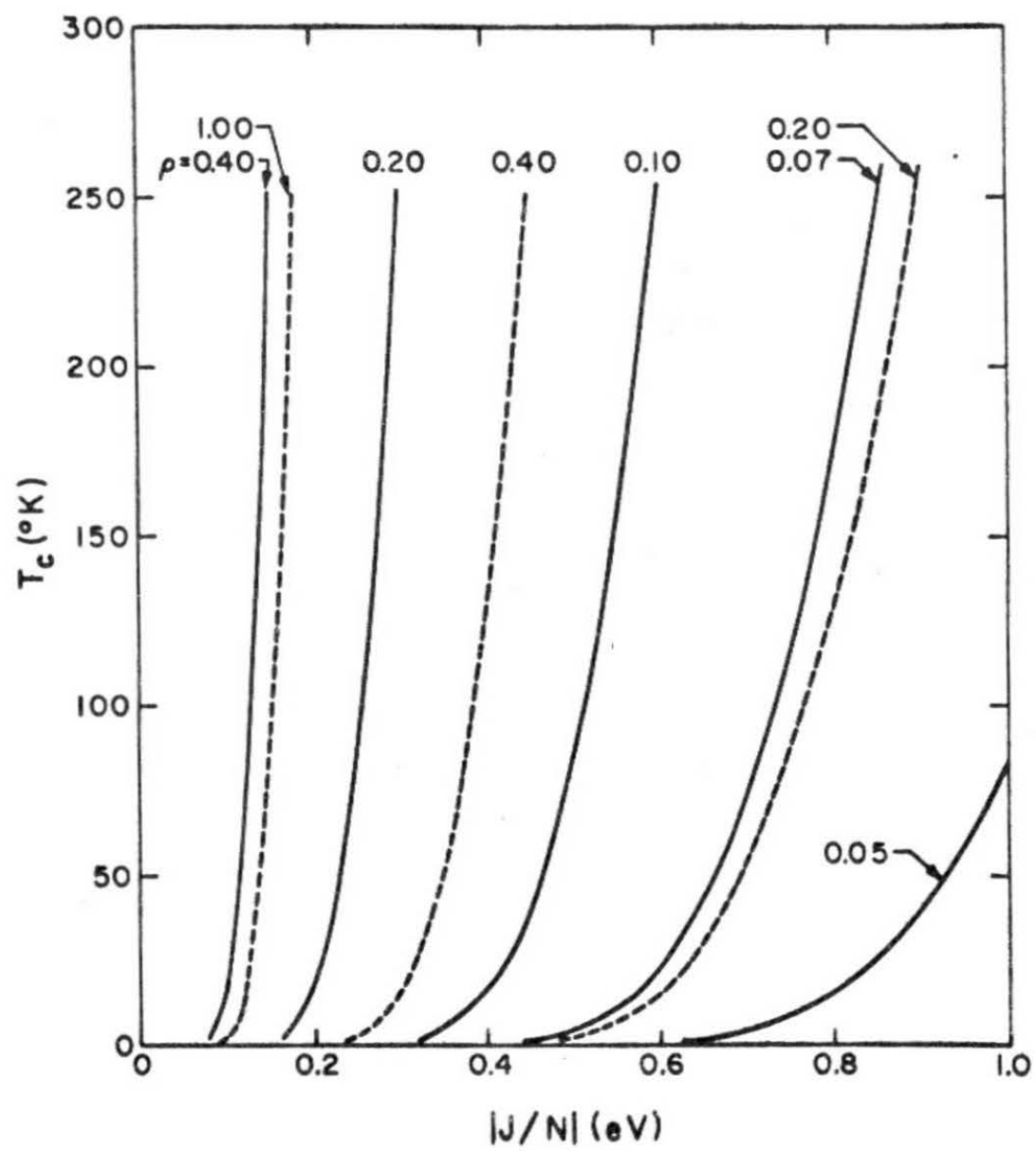

Fig. 10 Critical temperature against exchange interaction strength. Solid and dashed curves correspond to $\mathrm{J}\langle 0$ and $\mathrm{J}>0$ respectively. 


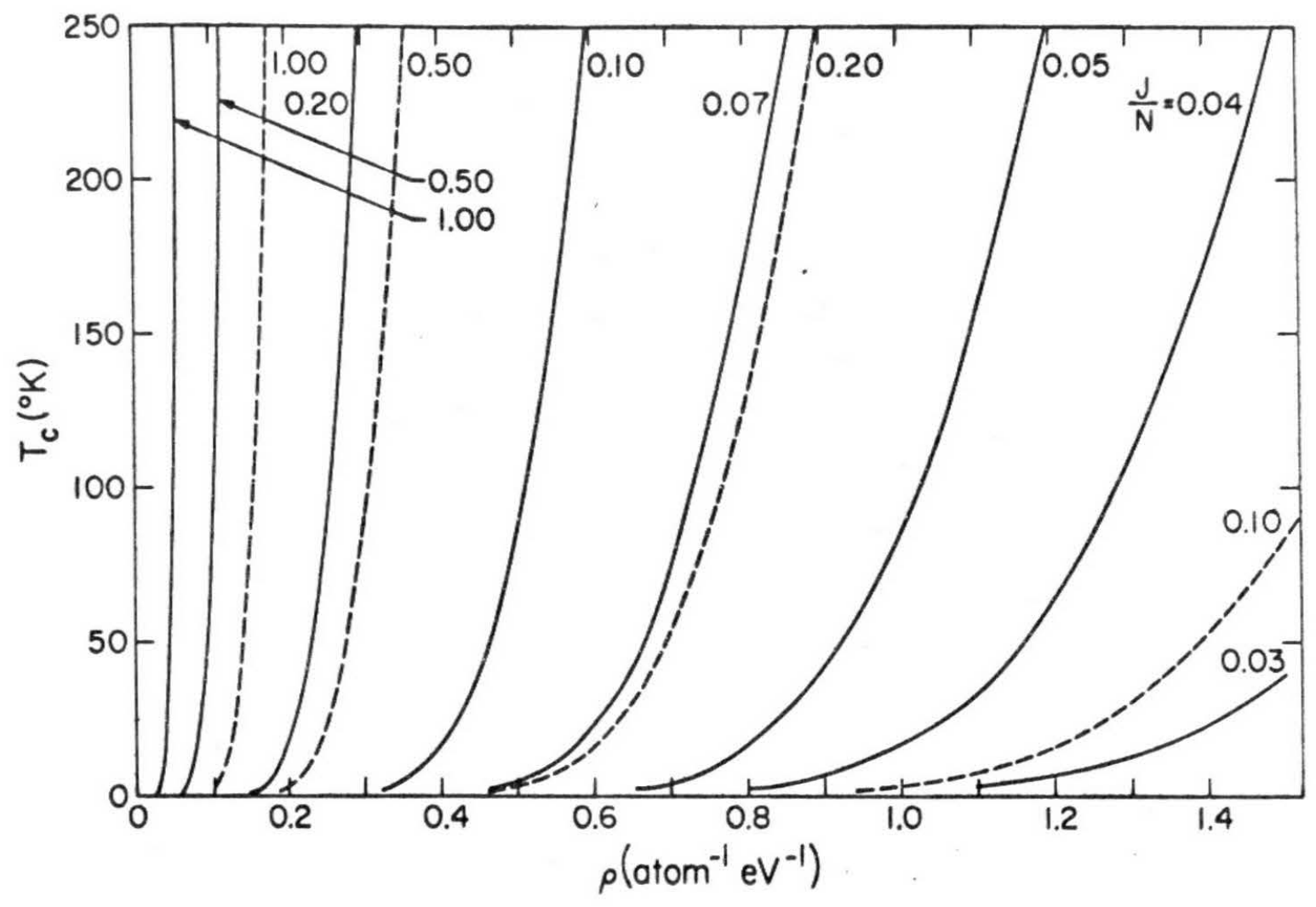

Fig. 11 Critical temperature against the density of states of the host. Solid and dashed curves represent the cases $\mathrm{J}\langle\mathrm{O}$ and $\mathrm{J}>\mathrm{O}$ respectively. 


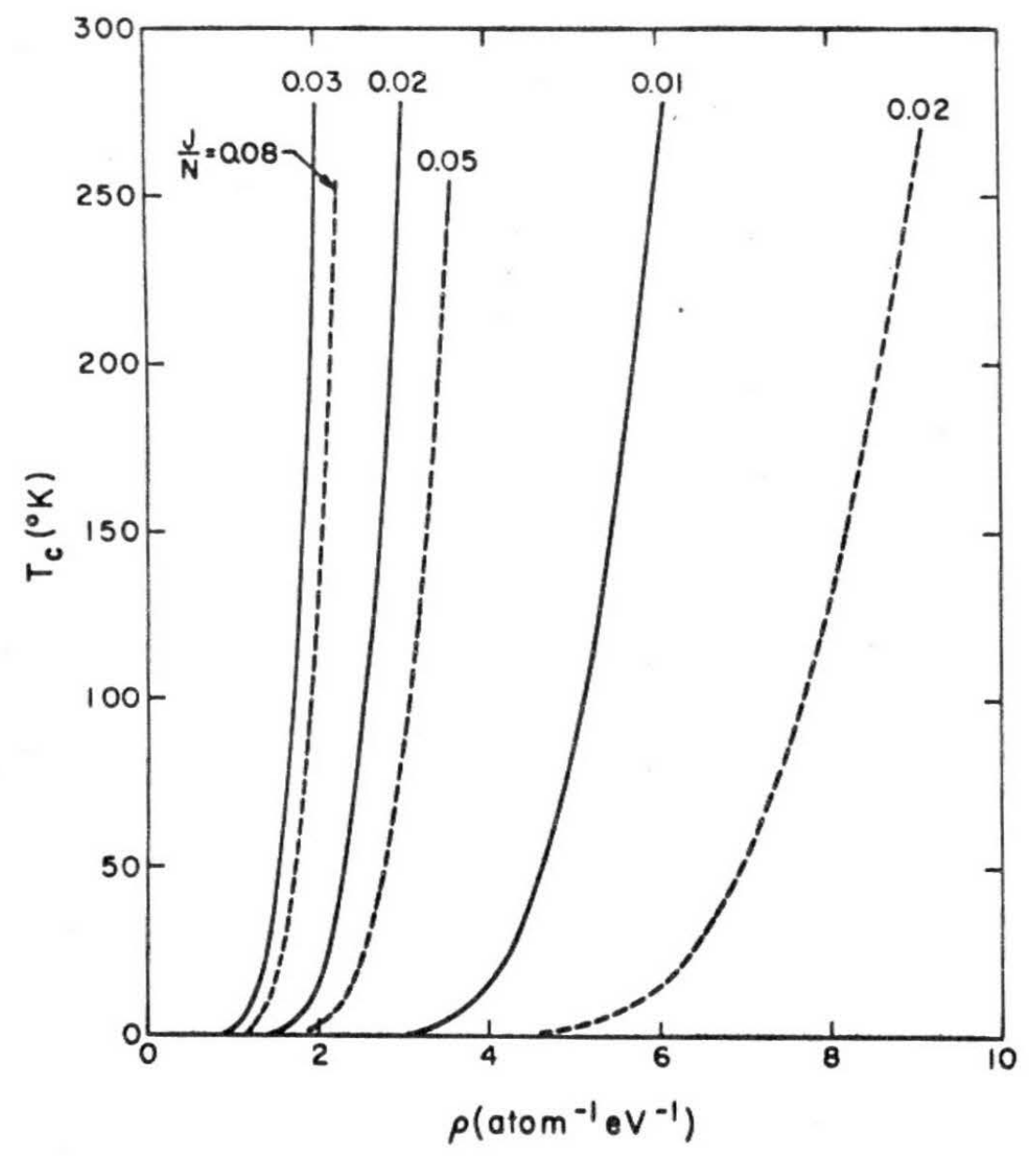

Fig. 12 Critical temperature against the density of states of the host. Solid and dashed curves correspond to $J<0$ and $J>0$ respectively. 


\section{ON DENSITY OF STATES}

From the work done on the single particle states in inter-

acting many particle systems, we know that the spectral density (42) of single particle states in the interacting system is given by,

$$
\begin{aligned}
A(\omega, k)= & -\pi^{-1} \operatorname{Im} \sum(\omega, k) \\
& \cdot\left[\left\{\omega-\epsilon_{k}-R_{e} \Sigma(\omega, k)\right\}^{2}\right. \\
& \left.+\left\{\lim \sum(\omega, k)\right\}^{2}\right]^{-1}
\end{aligned}
$$

(VII . 1)

where $\sum(\omega, k)$ is the self-energy of the electrons. We can make use of the spectral density to calculate the density of single particle states in the interacting system, for we have already obtained results for the self-energy $\Lambda(\omega, k)$. In terms of the spectral density, the density of states is given by,

$$
\rho(\omega)=\rho_{0}(\omega)+\int \rho_{0}\left(\epsilon_{k}\right) A(\omega, k) d \epsilon_{x}
$$

(VII .2) where $\rho_{0}(\omega)$ is the density of state function of the non-interacting system. For the present problem then $\rho(\omega)$ correspond s to the density of states of the magnetic alloy while $p_{0}(\omega)$ character- 
izes the density of states of the pure host.

As we mentioned in sec. III of the text, for "dilute" magnetic alloys, all the theoretical expressions for various quantities obtained from the idealized theoretical model of a single magnetic impurity interacting with the conduction electrons, are simply multiplied by the impurity concentration, c, to calculate physically observable parameters. Conversely one might consider this to be a limit on the term "dilute". Thus, to lowest order in impurity concentration,

$$
\sum(\omega, k)=c \Lambda(\omega, k)
$$

(VII • 3)

Where $\Lambda(\omega, k)$ is the self-energy we obtained from our single impurity model. We have already seen that our interaction $\mathrm{H}_{\mathrm{S}-\mathrm{d}}$ being a contact interaction, the scattering amplitude, and hence the self-energy $\Lambda(\omega, k)$ are independent of the momentum, $K$, of the electron, giving $\Lambda(\omega, k)=\Lambda(\omega)$. However, it is dependent on the temperature and thus we write it as $\Lambda(\omega, T)$. From equation (VII. 2) we then obtain the change in density of states as,

$$
\Delta \rho(\omega, T)=\rho(\omega, T)-\rho_{0}(\omega)
$$




$$
\begin{aligned}
= & \int_{-D}^{D} \rho_{0}\left(\epsilon_{k}\right) \cdot \pi^{-1}[\{-c \cdot \ln \Lambda(\omega, T)\} \\
& \left.\cdot\left[\left\{\omega-\epsilon_{k}-\operatorname{Re} \Lambda(\omega, T) \cdot c\right\}^{2}+\{c \cdot \ln \Lambda(\omega, T)\}^{2}\right]\right]
\end{aligned}
$$

(VII. 4)

For consistency, let us evaluate this integral for a constant density of states of the host and let us as before take this to be the value at the fermi energy. We obtain the result,

$$
\begin{aligned}
\Delta \rho(\omega, T)= & \pi^{-1} \cdot \rho_{0}\left(\epsilon_{F}\right) \cdot \tan ^{-1}[2 D C \sin \Lambda(\omega, T) \\
& \left.\cdot\left\{D^{2}-c^{2}\left(\Lambda_{R}^{2}(\omega, T)+\Lambda_{I}^{2}(\omega, T)\right)\right\}^{-1}\right]
\end{aligned}
$$

(VII. 5)

where $\Lambda_{R}$ and $\Lambda_{I}$ characterize the real and imaginary parts of $\Lambda$. We recall immediately that $\Lambda_{I}(\omega, T)$ is positive for all energies and temperatures since the inverse of $A_{I}$ gives the life time of the single particle states. This is also seen from the numerically evaluated $\Lambda_{I}$, plotted in Figs. $(5,6)$. Hence $\Delta S(\omega, \tau)$ is always positive. Physically, then, it is simply saying that the introduction of the magnetic impurities result $s$ in the creation of 
additional electronic states.

This increase in the density of states is extremely sensitive to changes of energy and temperature because of the rapid variation of $\Lambda_{I}(\omega, T)$ with energy and temperature. In general, the magnitude of the argument of arc tan on the R. H.S. of the expression for $\Delta \rho(\omega, T)$, equation (VII. 5), is small so that as a first approximation one may say that $\left[\Delta \rho(\omega, T) / \rho_{0}\left(\epsilon_{F}\right)\right]$ the change in density of states normalized with respect to the density of states of the host, is proportional to the impurity concentration. Hence for usual dilute magnetic alloys where the impurity concentration is of the order of $10^{-3}$, the magnitude of the change in density of states would also be small, and as such would require great efforts on the part of the experimentalist to measure it. However, one can, we believe, detect the rapid variation of $\left(\Delta \rho / \rho_{\bullet}\right)$ with temperature and energy. 


\section{INTERPRETATION OF THE RESULTS}

\section{A. Long Rang e Ordering}

In section ( V.C) we explained the physical origin of $\Lambda(\omega, T)$ and provided an interpretation for it as an effective complex potential under whose influence the particles are moving. This effective potential we have seen to be energy dependent and consequently characterizes an effective interaction which is nonlocal in time. We know that "bare" particles can interact instantaneously only while "quasi-particles" can have retarded interactions. Thus we find that a simple and natural outcome of our theoretical formalism is the very physical interpretation of the "ideal" problem of scattering of bare electrons off localized magnetic mome nts in terms of the "realistic" situation wherein "dressed" or "quasi-particles" are moving under the influence of an effective non-local complex potential.

The non-local character of the interaction is of great significance because it provides the essential mechanism by which even a short range interaction can give rise to long range effects. To under stand this phenomenon, let us consider the scattering process of a particle. We have seen during the course of our analysis of the scattering equations that a given particle can scatter repeatedly during the course of its motion. It is ther efore important to realize that in a multiple scattering process a particle can travel over distances much larger than the range of the interaction. Thus for quasi-particles of reasonably large life times, which we 
know to be the case for energies near the fermi energy, the effect of non local interaction can persist over distances much larger than the range of the interaction. Consequently, information can be carried in the system over large distances. Thus, even though in the present case, the exchange scattering of conduction electrons off localized magnetic moments was being characterized by a "contact" interaction (i. e., zero range interaction) the occurrence of non-local effective potential is telling us that our system is experiencing long range effects. We can readily understand this phenomenon if we consider the spin polarization of the conduction electrons caused by the magnetic impurity. We have already seen that the temperature at which $\Lambda_{R}(\omega, T)$ becomes zero corresponds to a resonant state being formed by a strong clustering of the conduction electrons around the magnetic impurity with their spins aligned antiparallel to the impurity spin for the case $\mathrm{J}<0$, the Kondo system, and with their spins parallel to the impurity spin for $J>0$, the case we would henceforth call the "ferromagnetic case." Below the se temperatures the potential becomes attractive, Figs. $(3)$ and $(4)$, thus confirming the formation of clusters. The se resonant states have a finite life time and thus particles come in the go out of the clusters. Since, as we just mentioned, the se particles can carry information over distances large as compared to the range of interaction, we find that in the realistic system of more than one magnetic impurity atom, the two clusters can effectively interact with each other by exchanging real particles. 
Furthermore, such long range interactions would be of considerable significance since the probability for all the particles to cluster within the range of interaction at the same time is extremely small in general, and in particular; for zero range interaction (of type $\mathrm{H}_{\text {s-d }}$ e.g.) cannot make sense.

In this way we then find that an indirect long range correlation between the impurity spins can be set up due to the polarization of the conduction electrons by the zero range $s-d$ exchange interaction. This long range indirect correlation between the impurity atoms would increase with increasing impurity concentration. The point at which the long range correlation effects become important enough to bring about some kind of interlocking of the impurity spins themselves, so that the motion of the impurity spins cannot be considered to be independent of each other anymore, we believe to be the critical concentration. In terms of this picture then, we can understand the observation that the critical impurity concentration is higher in the amorphous state than in the crystalline state of the same system. The electronic mean free path is much shorter in the amorphous state as opposed to the crystalline state. Consequently, we expect the electron to be able to carry the information of the state of a given impurity spin to shorter distances on the average before it loses the information due to its finite life time now. There is therefore a less probability of transfer of information of the state of one impurity atom to another through the electrons in the amorphous state as opposed t o crystalline 
state of the same material. Hence the critical concentration in the amorphous state would be higher than the crystalline case.

\section{B. The Ground State}

A further point of interest concerns the interpretation of the resonant state formed in the Kondo problem. We have seen in our formalism that the resonant state is a consequence of the effective potential itself becoming large and attractive below a certain critical temperature. This we believe to be a more appropriate interpretation, rather than its earlier interpretation as being a consequence of the Pauli exclusion principle effect of the kind involved in superconductivity. ${ }^{(24)}$ The controversial theoretical question of whether or not one is to expect a many-body condensed state for the magnetic impurity problem has been quite clearly answered by us. Within the lowest order approximation for evaluatin of the energy correction to the non-interacting ground state, we have shown the existence of a non-analytic binding energy for both, antiferromagnetic $(J<0)$ and ferromagnetic $(J>0)$ coupling $s$ in the limit of temperature going to zero. Such results were obtained by variational treatments of the problem using singlet or triplet many-body wave functions as well as by other approaches. However, because of the approximations involved in the se treatment $s$ and the divergent behavior of resulting physical parameters such treatments were no solution of the Kondo problem in as much as they raised the question of the effects of particle + hole excita- 
tion $s$ in the system. The self consistent evaluation of the self energy from our transcendental equation continues to show the presence of a finite energy correction in the limit of temperature going to zero, both for $J<0$ and $J>0$. This result, for $J<0$, has been conjectured before, but for $J>0$ it is believed that no such binding ene rgy occurs when multiparticle intermediate states are taken into account. Our results show that this belief is incorrect.

The occurrence of these binding energies does not however imply a sharp phase transition (at the critical temperature for the present problem), of the kind observed in a normal state going to the superconducting state. Although, as we pointed out in section ( VI. A) dealing with the evaluation of self energy, approximating the exact integral equation for self-energy by a transcendental equation eliminates any cooperative phenomenon, we believe our present results for the ground state are correct, for such a cooperative phenomenon is virtually impossible in a system like ours with very small number of degrees of freedom.

a gradual break up of the spin correlations with increasing temperature, which is confirmed by the decreasing nature of the self-energy at temperatures near the critical temperature. This theoretical nature of our result is in perfect agreement with the observed experimental results. NMR studies ${ }^{(45)}$ on various systems like $\mathrm{Cu} \mathrm{Cr}, \mathrm{CuFe}$ etc., have shown that the condensation to many body state occurs gradually as temperature is lowered so that the 
correlated state has not fully formed until $\mathrm{T}<<\mathrm{T}_{\mathrm{k}}$.

The binding energies we have obtained he re are to be interpreted as the energy difference between ground states containing a "bound" state and a ground state which is normal and has a $(2 \mathrm{~S}+1)$ fold spin degeneracy. However, we do not interpret these ground states as forming a singlet or triplet (for $\mathrm{J}<\mathrm{o}$ and $\mathrm{J}>\mathrm{o}$ respectively) with the localized moment, for we have shown them to be a consequence of the many-body nature of the conduction electron spin polarization around the impurity. Rather, we regard these states as ones arising out of resonant states being formed in the total angular momentum channels $\mathrm{j}=\mathrm{S} \pm \frac{1}{2}$ for $\mathrm{J}>\mathrm{o}$ and $\mathrm{J}<\mathrm{o}$ respectively. Even for the simple case of $S=\frac{1}{2}$ we believe it is erroneous to interpret these states as being formed by a conduction electron being bound to the localized spin with its spin aligned parallel or antiparallel. Consequently the use of the terminology singlet or triplet is not only confusing but clearly undermines the true many-body nature of the ground state of the magnetic impurity + conduction electron system.

\section{Negative J vs Positive J: Resolution of an Apparent}

\section{Contradiction}

We would like to point out that although our results give a non-analytic binding energy for both antiferromagnetic and ferromagnetic exchange interactions, there are differences worth noting. First of all, we notice that one cannot obtain the energy correction 
to the ground state of the problem for one case by replacing $\mathrm{J}$ by $-J$ in the result for the other, at any temperature. The treatment of the problem within other theories has lead people to believe ${ }^{(5)}$ that one can do so for temperatures above the Kondo temperature (the critical temperature for $J<0$ in our case), as their results do not show a critical temperature for $\mathrm{J}>$ o. Below the Kondo temperature however, because of the occurrence of a binding energy for $\mathrm{J}<0$, it was recognized that one cannot obtain results for one case from the other by a mere replacement of $\mathrm{J}$ by $-J$.

Furthermore, for the same value of the critical temperatures in both the cases our results show that one requires a much higher magnitude of the exchange interaction strength, $|\mathrm{J}|$, for ferromagnetic coupling as compared to antiferromagnetic coupling (Fig. 7). The same is reflected in the binding energy at absolute zero, where we find that it is higher for antiferromagnetic coupling, for the same magnitude of the exchange interaction strength. However, the higher magnitude of $J$ required for ferromagnetic coupling $(\mathrm{J}>\mathrm{o})$ is definitely not, unreasonable, for in systems with $\mathrm{J}>0$ e.g. $\mathrm{Fe}, \mathrm{Co}$ in Pd, the polarization of the d-electrons of the host by the impurity enhances the effective magnitude of the exchange interaction considerably. Consequently the effective strength of the exchange interaction as felt by the s electrons of the conduction band is much higher than the original bare $\mathrm{J}$ value. This enhancement of the moment is further increased by the polarization of the $s$ electrons 
themselves resulting in giant moments observed in such systems.

The existence of a critical temperature for $J>0$ is of great significance. Cottet et al. ${ }^{(50)}$ have observed the simultaneous existence of giant moments and a Curie-Weiss behavior of susceptibility in $\mathrm{Ni}_{1-\mathrm{x}^{\mathrm{Rh}}}$ host with $\mathrm{Fe}$ as impurity, for $\mathrm{Rh}$ concentration, $\mathrm{x}$, above $38 \%$. The existence of a giant moment, along with the observed absence of resistivity minimum, requires $\mathrm{J}>$ o. On the othe $\mathrm{r}$ hand, the susceptibility behavior, if inter preted in terms of the existing theories of Kondo systems requires $J<0$. This clearly shows that the critical temperature obtained from the susceptibility behavior cannot be regarded as a Kondo temperature, which exists only for $J<0$. We resolve this apparent contradiction by interpreting this critical temperature as the one corresponding to $\mathrm{J}>0$ in our theory.

The observed linear increase of this critical temperature with increasing $\mathrm{Rh}$ content can qualitatively be understood by putting together the facts that the magnetic moment is decreasing while the density of states is increasing almost linearly, information obtained from band structure of $R h$, as we put in more and more of $R h$ in Ni. The reduction in magnetic moment implies a reduction in the effective $\mathrm{J}$ value. A look at the behavior of the critical temperature for $J>0$ as a function of the density of states, $\rho$, for fixed values of $\mathrm{J}$ (Figs. 11, 12) clearly shows that if we let $\mathrm{J}$ decrease as $p$ increases, then for the right combination of $J$ and $\rho$ a linear increase in $T_{c}$ with $p$ is possible. 
A direct quantitative comparison of our theoretical results with experimental results must unfortunately await progress in experimental results on various quantities involved. 


\section{ON PHYSICAL PARAMETERS OF KONDO SYSTEMS}

As mentioned in the introduction, the consequences of the magnetic impurity-conduction electron interaction manifest themselves in various anomalous behavior $\mathrm{s}$ in the physical parameter $\mathrm{s}$ of the dilute magnetic alloy. (see Fig. 1) No theory exists today which can account for these behaviors in a complete and comprehensive way. We have for the first time been able to consider the contribution of (particle + hole) excitations in the theoretical treatment of the problem and have shown that the resulting solutions are free of unphysical singularities. One could now make use of our results of the electron self-energy to calculate these physical parameters. Unfortunately, because of lack of explicit analytic expressions for the self-energy, one would have to resort to a numerical study of these parameter $\mathrm{s}$.

Since the aim of the present work was primarily to be able to provide a theoretical formalism which could in a simple way incorporate the multiparticle intermediate states, thus enabling us to answer the most open question of the ground state of the magnetic impurity conduction electron system, we have not performed a numerical study of the physical parameters. However, we would discuss them briefly on the basis of our theoretical results in the following pages, in so far as they shed some light on the ground state of the problem.

A. Resistivity and Thermoelectric Power

Of all the physical parameters calculated from the existing 
theoretical formalism, there is a general consensus on the resistivity approaching and remaining at the unitarity limit at low temperatures, with a logarithmic variation with temperature in the region near the Kondo temperature. Similarly, there is general agreement on the giant thermo-electric power. Consequently, the transport properties at low temperatures do not seem to provide a critical test of the proper low temperature treatment of the problem. On the other hand, specific heat does seem to provide such a test. Our main interest here being in the experimental results on physical parameters which can provide suitable information on the ground state of the system so that we can check our theoretical results and their interpretation, we discuss resistivity and thermoelectric power very briefly and shall go into specific heat at some length.

We showed in section (VI. A) that the second order expansion in powers of $\mathrm{J}$ of the first iterative solution for the electron selfenergy is the same as obtained by Doniach ${ }^{(20)}$ for what he calls effective potential in his diagram summation formalism. Doniach(20) has performed a numerical study of the electrical resistivity and thermoelectric power using these results and compared them with the experimental results on various systems (e.g. $\mathrm{Rh} \mathrm{Fe}, \mathrm{Pd} \mathrm{Fe}$ etc.). He finds an extremely good agreement between the theoretical and experimental results. It is to be noted that the results obtained by using the expressions (VI. 11) and (VI. 12) for the electron selfenergy are already a considerable improvement over Kondo 's 
original expression. Furthermore, under appropriate approximations these results reduce to the Kondo results with the famous logarithmic dependence of resistivity on temperature. Thus we find that even expressions (VI. 11) and (VI. 12) for the electron selfenergy provide the correct behavior of electrical resistivity and thermoelectric power.

However, the main point of interest for us is understanding the mechanism behind the observed flattening of resistivity at very low temperatures, for the case of $\mathrm{J}<0$. A popular explanation for this phenomenon is based on the concept of "spin compensation" of the magnetic impurity spin by the antiparallel spin polarization of the conduction electrons. The idea is that because of the spin compensation of the magnetic moment there is no more a chance for electrons to see the magnetic moment and hence no Kondo scattering. If this were really the mechanism, one should observe a quenched moment in other experiments also e.g. magnetic susceptibility. Unfortunately this has almost never been the case. Hence this mechanism cannot be solely responsible for flattening of the resistivity.

One can understand the flattening of resistivity without a complete compensation of the magnetic impurity spin if one looks into the factors responsible for the magnitude of transition probability which is directly related to the resistivity. The transition probability not only depends on the magnitude of the matrix elements of the interaction but also on the density of states of the final states. Thus the reduction in the rate of increase of resistivity and its 
flattening off not only depends upon the reduction of the effective magnetic moment through spin compensation but also on the behavior of the density of states as the temperature is lowered. From the expression for the incremental density of states, equation (VIII. 5), we find that it essentially behaves like $\Lambda_{\mathrm{I}}(\mathrm{T})$. Fig. (5) for $\Lambda_{I}(T)$ clearly shows that it reaches its peak value near the Kondo temperature and then decreases as the temperature is further lowered. Thus we find that the density of stat es itself decreases with decrease in temperature below the Kondo temperature, thereby supressing the rate of increase of the resistivity. Thus we see that the flattening of resistivity must necessarily be considered as the combined effect of reduction of the magnetic moment and the density of states. Previous attempts to explain it on the basis of reduction of magnetic moment alone not only lead to conceptual contradictions and are inadequate, but also miss an as fundamental a mechanism, namely the behavior of density of states.

\section{B. Specific Heat}

As opposed to transport properties, thermodynamic properties like specific heat apparently provide better information on the ground state of the system. As such they seem to provide a better test for the existing theories. Since the energy of a system is such a fundamental quantity, specific heat measurements are expected to reveal much about the formation of the resonant state and consequently the ground state of the system. Unfortunately, 
arriving at numerical values for specific heat based on its definition involves more uncertainty than any other physical parameter. This is so for two essential reasons. First, the measurements must all be performed at extremely low temperatures e.g. for a typical Kondo system like Cu Fe measurements below $1^{\circ} \mathrm{K}$ are considered essential to obtain any worthwhile information. Second, the impurity concentration must be high so that the incremental specific heat is not completely over shadowed by the host specific heat. This latter requirement has in the past always cast doubt on the observed specific heat data for it is hard to be free of impurity-impurity interactions for the high impurity concentrations involved in these experiments. Thus in the past any observed deviations in specific heat behavior from those predicted by the existing approximate theories of the $s-d$ exchange scattering have been associated with the impurity-impurity interactions. However, even for impurity concentrations in the range where impurityimpurity interactions are shown to be absent, specific data show behavior which are outside the scope of the existing results. We show later on in this section that such departures can be explained by our results.

Before we present the details of the calculations of specific heat let us consider qualitatively the general features of a typical specific heat curve (Fig. 1) in light of our theoretical results for electron self-energy. We provided an interpretation for $\wedge_{R}$ (Fig. 3) as an effective potential. The temperature at which it becomes 
zerowe saw leads to the onset of a resonant state. Below this temperature we find that the resonant state becomes more and more strongly bound. However, $\Lambda_{R}(T)$ instead of monotonically increasing in magnitude with lowering in temperature, reaches a maximum value and then turns around. Thus we find that the resonant state is most strongly bound at some temperature between zero and the critical temperature. Consequently, one should observe a peak in the specific heat around this temperature as sociated with the thermal destruction of the resonant state. This then qualitatively and physically explains the typical behavior of specific heat (Fig. 1.).

Let us now turn to a more quantitative and rigorous discussion of the specific heat. For this we have to calculate the total change in the energy of the system which is given by the well known result,

$$
\Delta E=\int \omega \cdot \Delta \rho(\omega, T) f(\omega, T) d \omega
$$

In fact $(\triangle E)$ represents the change in the free energy of the system if the single particle energies are measured with respect to the Fermi energy. $\Delta p(\omega, T)$ and $f(\omega, T)$ refer to the incremental density of states of the conduction electron + impurity system and the Fermi distribution function respectively. The incremental specific heat is then given by, 


$$
\Delta C=\frac{\partial}{\partial T}\{\Delta E(T)\}
$$

(IX. 2)

To get an explicit curve for $\Delta C$ one could evaluate $\Delta E$ by brute force, numerically, and then its temperature derivative. However, we can extract all the interesting information on the behavior of the incremental specific heat without having to resort to such a numerical computation.

We can evaluate $\Delta E$ by considering the following integral,

$$
I=\int_{0}^{\infty} f(\omega, T)\left\{\frac{d}{d \omega} F(\omega, T)\right\} \cdot d \omega
$$

where $F(\omega, T)$ is such a function of the energy $\omega$ that as $\omega \rightarrow 0$, $F(\omega, T) \rightarrow$ o. Expanding $F(\omega, T)$ about the Fermi energy we have,

$$
\begin{aligned}
F(\omega, T)=\left[F\left(\epsilon_{F}, T\right)\right. & +\left(\omega-\epsilon_{F}\right) F^{\prime}\left(\epsilon_{F}, T\right) \\
& \left.+\frac{1}{2}\left(\omega-\epsilon_{F}\right)^{2} F^{\prime \prime}\left(\epsilon_{P}, T\right)+\cdots\right]
\end{aligned}
$$


where $F^{\prime}, F^{\prime \prime}$ etc. refer to the first, second, derivatives of the function $F$ with respect to

Now, from (IX.3) we have,

$$
\begin{aligned}
I= & {\left[\int f(\omega, T) F(\omega, T) d \omega\right]_{0}^{\infty} } \\
& -\int_{0}^{\infty} F(\omega, T)\left\{\frac{d}{d \omega} f(\omega, T)\right\} \cdot d \omega
\end{aligned}
$$

(IX. 5)

The first term on the R.H.S. of (IX. 5) is zero since $\lim \omega \rightarrow \infty$ of $f(\omega, T)=0$ and $\lim \omega \rightarrow 0$ of $F(\omega, T)=0$. Therefore,

$$
I=-\int_{0}^{\infty} F(\omega, T)\left\{\frac{d}{d \omega} f(\omega, T)\right\} d \omega
$$

(IX. 6)

Substituting for $F(\omega, T)$ from (IX. 4) in (IX. 6) we obtain,

$$
I=-\int_{0}^{\infty}\left[F\left(\epsilon_{F, T}\right)+\left(\omega-\epsilon_{F}\right) F^{\prime}\left(\epsilon_{F}, T\right)+\right.
$$


$-152-$

$$
\left.\frac{1}{2}\left(\omega-\epsilon_{F}\right)^{2} F^{\prime \prime}\left(\epsilon_{F}, T\right)+--\cdot\right] \cdot\left\{\frac{d}{d \omega} f(\omega, T)\right\} \cdot d \omega
$$

Changing the origin to Fermi energy in the integral, the lower limit changes from 0 to $\left(-\epsilon_{F} / k_{B} T\right)$, which for the very low temperatures, $k_{B} \cdot T<<\epsilon_{F}$ may be replaced by $-\infty$. Thus we have,

$$
\begin{aligned}
I=\left[L_{0} F\left(\epsilon_{F,} T\right)\right. & +L_{1} F^{\prime}\left(\epsilon_{F, T}\right) \\
& \left.+L_{2} F^{\prime \prime}\left(\epsilon_{F}, T\right)+\cdots \cdot\right]
\end{aligned}
$$

(IX. 7)

where,

$$
\begin{aligned}
L_{0} & =-\int_{-\infty}^{\infty} \frac{d}{d \omega} f(\omega, T) \cdot d \omega=1 \\
L_{1} & =-\int_{-\infty}^{\infty}\left(\omega-\epsilon_{F}\right) \frac{d}{d \omega} f(\omega, T) \cdot d \omega \\
& =0, \quad \text { since }\left(\frac{d f}{d \omega}\right) \text { is even }
\end{aligned}
$$

and

$$
L_{2}=-\frac{1}{2} \int_{-\infty}^{\infty}\left(\omega-\epsilon_{F}\right)^{2} \frac{d}{d \omega} f(\omega, T) \cdot d \omega=\frac{\pi^{2}}{6}\left(k_{B} T\right)^{2}
$$


Let us now identify $F^{\prime}(\omega, T)$ with $\{\omega \cdot \Delta \rho(\omega, T)\}$ occurring in the integral for $\Delta \mathrm{E}$, equation (IX. 1). From the section on density of states we find that $\Delta S(\omega, T)$ behaves essentially like $n_{I}(\omega, T)$ which goes to a constant value as $\omega$ goes to the Fermi energy. Also,

$$
F(\omega, T)=\int \omega \cdot \Delta \rho(\omega, T) d \omega
$$

Thus we expect $F(\omega, T)$ defined by (IX.8) to go to zero as $\omega$ goes to zero. (It can at the most go to a constant value if a peculiar cancellation in the rate change of $\omega$ and $\Delta \rho(\omega, T)$ takes place). Combining (IX. 8) with (IX. 1) we obtain by the standard method given by Mott and Jones,

$$
\Delta E=\frac{\pi^{2}}{12}\left(k_{B} T\right)^{2} \cdot\left[\frac{d}{d \omega}\{\omega \cdot \Delta \rho(\omega, T)\}\right]_{\omega=\epsilon_{F}}
$$

(IX. 9)

where we have retained terms only up to $L_{2}$ in (IX. 7). Using (IX.2) we obtain the incremental specific heat as, 


$$
\begin{aligned}
\Delta C= & {\left[\frac{\pi^{2}}{12}\left(k_{B} T\right)^{2} \frac{\partial}{\partial T} \Delta \rho\left(\epsilon_{F}, T\right)\right.} \\
& \left.+\frac{\pi^{2}}{6} k_{B}^{2} T \cdot \Delta \rho\left(\epsilon_{F}, T\right)\right]
\end{aligned}
$$

(IX.10)

Realizing that the incremental density of states is essentially given by,

$$
\Delta \rho\left(\epsilon_{F}, T\right) \approx\left\{\frac{2 \rho_{0}\left(\epsilon_{F}\right) \cdot c}{\pi D}\right\} \cdot \Lambda_{I}\left(\epsilon_{F}, T\right)
$$

(IX. 11)

we obtain from (IX. 10),

$$
\Delta C=\frac{\pi \rho_{0}\left(\epsilon_{F}\right) k_{B}^{2} \cdot c}{3 D}\left[\frac{1}{2} T^{2} \frac{\partial}{\partial T} \Lambda_{I}\left(\epsilon_{F}, T\right)+T \Lambda_{I}\left(\epsilon_{F}, T\right)\right]
$$

(IX.12)

Let us call the first and second terms on the R.H.S. $\Delta C_{1}$ 
and $\quad \Delta C_{2}$. Then from Fig. (5) we find their relative magnitude, $\left(\Delta C_{1} / \Delta C_{2}\right)$, for temperatures in the range of $1^{\circ} \mathrm{K}$, as,

$$
\begin{aligned}
\frac{\Delta C_{1}}{\Delta C_{2}} & =\frac{T \frac{\partial}{\partial T} \Lambda_{I}\left(\epsilon_{F}, T\right)}{\Lambda_{I}\left(\epsilon_{F}, T\right)} \\
& \approx 10^{-1}
\end{aligned}
$$

To consider the temperature dependence of the incremental specific heat let us analyze the two terms on the R. H. S. of (IX. 12) separately. To start with, it is important to realize that even though $\Lambda_{I}\left(\epsilon_{F}, T\right)$ as a function of temperature, plotted in Fig. 5 shows a dip as tempe rature increases from zero with a maximum near the critical temperature, it is not the cause of the observed anomalous behavior of the incremental specific heat shown in Fig. 1. This is confirmed by a monotomic increase in $T \cdot \Lambda_{I}\left(\epsilon_{F}, T\right)$ plotted against $T$ in Fig. (13). Thus we find that the second term in (IX.12), which correspond $s$ to the contribution of the incremental density of states at the Fermi energy, leads to a "normal" contribution to the incremental specific heat of the dilute magnetic alloy. As opposed to this, we find that the anomalous behavior of $\triangle \mathrm{C}$ is due to the first term in (IX, 12). 
As the first term explicitly shows, its origin lies in the rate of change of the incremental density of states at the Fermi energy with respect to the change in temperature. Since Fig. (5) for $\Lambda_{I}\left(G_{F}, T\right)$ shows that it reaches its maximum value very near the critical temperature and has a minimum at a temperature around one seventh of the critical temperature it's derivative with respect to temperature shall be zero at these two temperatures, reaching it's maximum value between $\frac{1}{7} \mathrm{~T}_{\mathrm{c}}$ and $\mathrm{T}_{\mathrm{c}}$. From Fig. (5), we find that this peak would occur between $\frac{1}{3} T_{c}$ and $\frac{1}{4} T_{c}$. This then clearly explains the anomalous behavior of incremental specific heat shown in Fig. 1.

It is important to mention at this point that in the past this anomalous behavior has generally been interpreted from the band theory point of view as due to the temperature variation of the incremental density of electronic states at the Fermi surface. The foregoing discussion of our result has unambiguously shown that this interpretation is incorrect. The origin of the anomalous part is in the temperature variation of the rate of change, with respect to temperature, of incremental density of electronic states.

Besides explaining the usual anomalous peak in incremental specific heat of the dilute magnetic alloy, our results predict another interesting phenomenon at extremely low temperatures. From our expression (IX.12) for $\Delta \mathrm{C}$ we find that its temperature variation is essentially the combined effect of the two terms $\Delta C_{1}$ and $\Delta C_{2}$. From (IX.12) and Fig. (5) for $\Lambda_{I}\left(t_{F}, T\right)$ we infer 


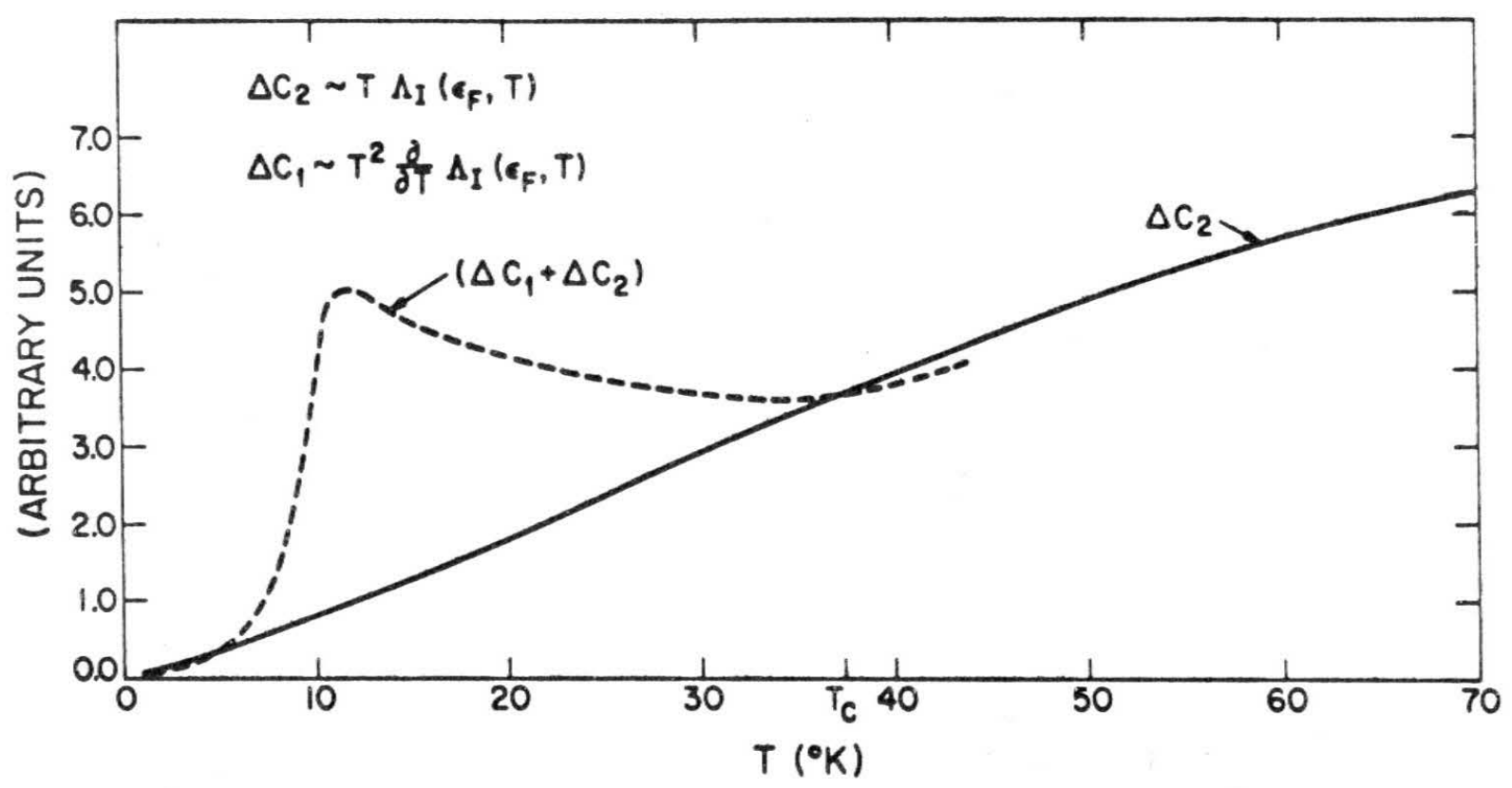

Fig. 13 Incremental specific heat as a function of temperature. $\Delta C_{1}$ and $\Delta C_{2}$ correspond to the contributions of the two terms of equation (IX.12). $\Delta C_{1}$ curve has been greatly exaggerated for the sake of clarity. 
that at extremely low temperatures $\Delta C_{1}$ shall tend to zero much faster than $\Delta \mathrm{C}_{2}$. From (IX.12) we know that $\Delta \mathrm{C}_{1}$ is of the order of $\left(\Delta C_{2} / 10\right)$. Thus below the lower temperature where $\Delta C_{1}$ is zero, it is negative but vanishingly small so that the temperature variation of $\Delta \mathrm{C}$ in this temperature region is essentially governed by $\Delta C_{2}$. Hence, if one plots $(\Delta C / T)$ against $\mathrm{T}$ one should expect a behavior governed essentially by $\left(\Delta \mathrm{C}_{2} / \mathrm{T}\right)$ which is the behavior of $\Lambda_{I}\left(\epsilon_{F}, T\right)$. Going back to Fig. (5) for $\Lambda_{I}\left(\epsilon_{F}, T\right)$ we find that in this temperature region it shows a rise as temperature is lowered. Thus our results predict a rise in $(\Delta \mathrm{C} / \mathrm{T})$ at temperatures much below the temperatures where the usual peak in the incremental specific heat is observed.

There are not many systems on which accurate measurements have been reported at temperatures in this range, which for our numerical results is around one tenth of the critical temperature. However, we do have results available on one of the most typical, and hence most extensively studied, of the Kondo systems, namely CuFe. Franck, Manchester and Martin ${ }^{(47)}$ have performed measurements of specific heat of the CuFe system, going to temperatures as low as $0.5^{\circ} \mathrm{K}$. These results have been analyzed by Heeger et al ${ }^{(45)}$ and they find a clear upturn in $(\Delta C / T)$ at temperature s below $1^{\circ} \mathrm{K}$. It is important to mention that the Kondo temperature of the CuFe system is taken to be $16^{\circ} \mathrm{K}$ and that the usual peak in incremental specific heat is observed around 
$5^{\circ} \mathrm{K}$, corresponding to the typical qualitative behavior shown in Fig. 1. The upturn in $(\Delta \mathrm{C} / \mathrm{T})$ below $\mathrm{l}^{\circ} \mathrm{K}$ is clearly separate from this and confirms the predictions of our results.

It is quite difficult to predict an explicit temperature dependence of $\triangle C$ from the numerically obtained curve for $\wedge_{I}\left(t_{F}, T\right)$ for all ranges of temperature. However, Fig. 5 plotted against $\ln \mathrm{T}$, shown in Fig. 14, shows a linear dependence in the temperature range of about $8^{\circ} \mathrm{K}$ to $25^{\circ} \mathrm{K}$. The critical temperature we remind is $37^{\circ} \mathrm{K}$. Expecting that this behavior shall remain unmodified for different values of the parameters $J$, impurity spin $S$ and density of states of the host $P_{0}$, we may infer that in the temperature range $0.3 T_{c}$ to $0.6 T_{c}, \Lambda_{I}\left(t_{f}, T\right)$ depends on temperature logarithmically. Thus from (IX.12) we find that in this temperature range $(\Delta C / T)$ shall essentially show the dependence,

$$
(\Delta C / T) \sim \ln T
$$

For temperatures around the minimum point of $\lambda_{I}\left(t_{F}, T\right)$ we have not been able to extract any explicit temperature dependence. In the extremely low temperature region, $\mathrm{T}<0.1^{\circ} \mathrm{K}$, our results show that the incremental specific heat $\Delta C$ shall tend to zero almost linearly with $T$. This we infer on the basis of the almost constant nature of $n_{I}\left(t_{P}, T\right)$ as temperature approaches these values in Fig. (14). The lowest temperature at which we have performed numerical evaluation is $l^{\circ} \mathrm{K}$ which is roughly $(1 / 40) \mathrm{T}_{\mathrm{c}^{\prime}}$, and 
$-160=$

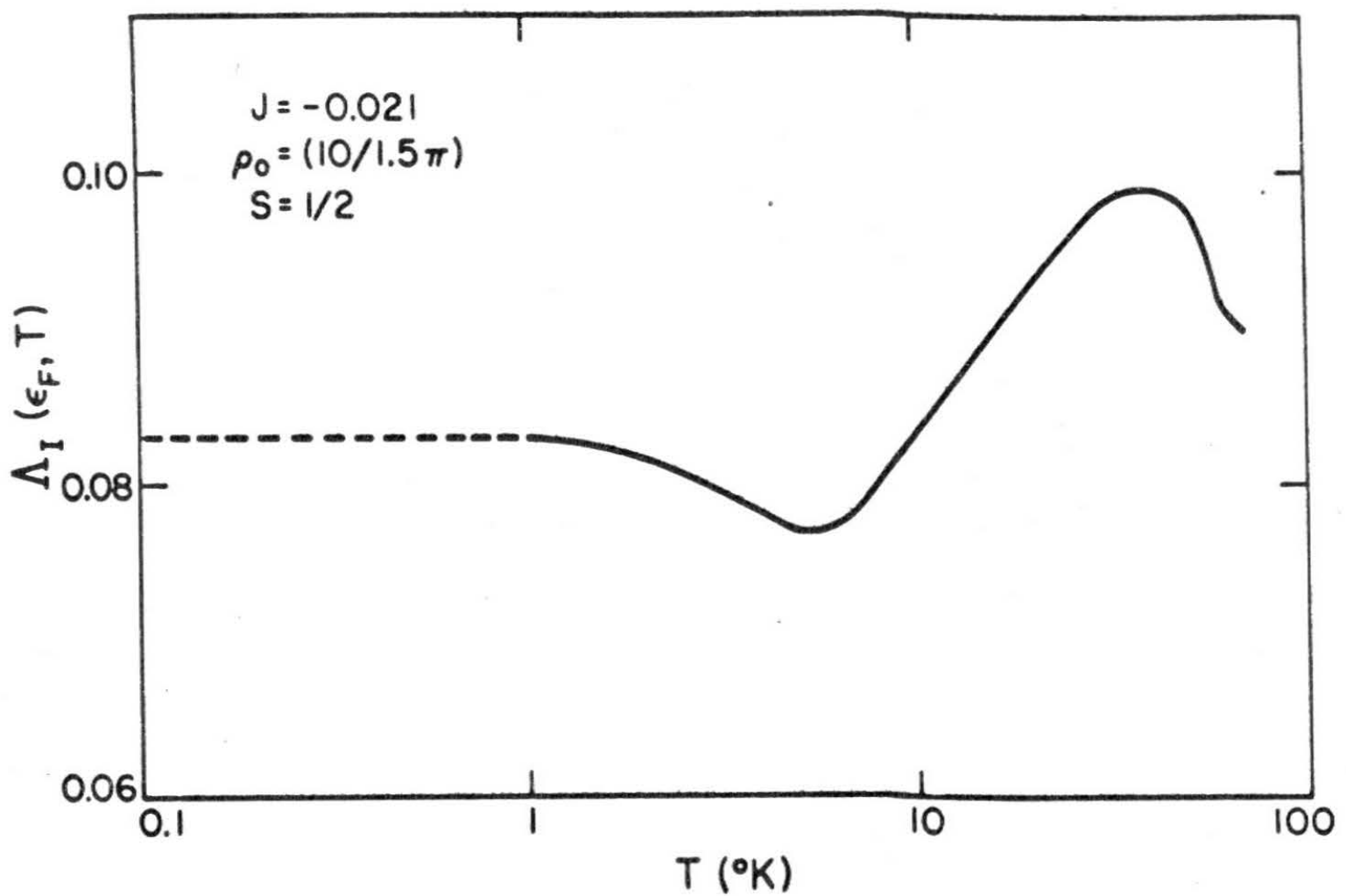

Fig. 14. $\Lambda_{I}\left(\epsilon_{F}, T\right)$ plotted against temperature on logarithmic scale. Note the linear depend-

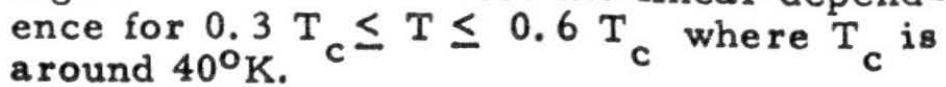


the curve below this temperature is extrapolation, anticipating no pecularities in the nume, rically evaluated, $n_{I}\left(f_{P}, T\right)$ in this region. Thus,

$$
\Delta \mathrm{C} \sim \mathrm{T}, \text { for } \mathrm{T} \leq 10^{-2} \mathrm{~T}_{\mathrm{c}}
$$

We conclude our discussion of the incremental specific heat of the dilute magnetic alloy by comparing our results with those provided by other theories for $T<<T_{c}$ and summarized in the table below;

Applebaum, Kondo $\quad \Delta \mathrm{C} \sim \mathrm{T} \ln \mathrm{T}$

Abrikosov, Suhl, Klein,

Levine

$\Delta \mathrm{C} \sim \mathrm{T}$

Bloomfield, Hamman,

$\Delta C \sim T^{0.57}$

Muleller-Hartmann,

Zittarz

$\Delta C \sim T^{0.57}$ and $\Delta C \sim(\ln T)^{-4}$

Ander son

$$
\text { for } \mathrm{T} \rightarrow 0
$$

$\Delta \mathrm{C} \sim \mathrm{T}^{\frac{1}{2}}$

Comparing these expressions with our results we find that the Applebaum-Kindo expression is valid not for all temperatures below $\mathrm{T}_{\mathrm{c}}$ but in a region approximately $0.2 \mathrm{~T}_{\mathrm{c}}$ to $0.6 \mathrm{~T}_{\mathrm{c}}$. In the limit $\mathrm{T} \rightarrow$ o our result appears to be closest to that of Abrikosov etc., showing an essentially linear dependence. Thus, we find that the previously obtained results are of limited validity and it would be exroneous to extend any one of them out of their 
temperature limits. As a final remark we mention that our result s (IX.13) and (IX.14) are in excellent agreement with the experimental results of typical Kondo systems like CuFe and $A u V_{0}^{(48,49)}$ Realizing that the Kondo temperatures for the se systems are around $15^{\circ} \mathrm{K}$ and $300^{\circ} \mathrm{K}$ respectively, we find that our results are indeed capable of describing the specific heat behavior over a wide range of temperatures. 


\section{APPENDIX 1}

We have already shown in the text that because of the bilinear nature of the interaction $\mathrm{H}_{8-\mathrm{d}}$, a given state of the system with $\mathrm{m}$ electrons knocked out of the background $\phi_{N}$, can be coupled only to states with $(m-1)$, mand $(m+1)$ electrons knocked out of $\phi_{N}$. Represented mathematically (in short but self evident notation) the scattering equations from a single particle to $(2 \mathrm{~m}+1)$ particle states would thus be of the form,

$$
\begin{aligned}
& \left\langle\phi_{N-m}, m, k^{\prime} \sigma^{\prime}, s_{z}^{\prime} \mid \psi_{k}\right\rangle= \\
& =\left[E_{k}-\left\{\left(\varepsilon_{0}+\epsilon_{k^{\prime}}+\epsilon_{k_{1}}+\cdots+\epsilon_{k_{m}}\right)-\left(\epsilon_{k_{1}}+\cdots-\epsilon_{k_{\beta_{m}}}\right)\right\}\right]^{-1} \\
& \cdot(-J / N)\left[\sum_{k^{\prime \prime} \sigma^{\prime \prime} s_{z}^{\prime \prime}}\left\langle\phi_{N-(m-1)}(m-1), k^{\prime \prime} \sigma_{j}^{\prime \prime} s_{Z}^{\prime \prime} \mid \psi_{k}\right\rangle\right. \\
& +\sum \sum \sum\left\langle\phi_{N-m}, m, k^{\prime \prime} \sigma^{\prime \prime} ; S_{z}{ }^{\prime \prime} \mid \psi_{\alpha}\right\rangle \\
& k^{\prime \prime} \sigma^{n} S_{\varepsilon}^{\prime \prime m} \\
& \left.+\sum_{k \cdot \sigma^{\prime \prime} S_{g}^{\prime \prime}} \sum_{(m+1)}\left\langle\phi_{N-(m+1)},(m+1), k^{\prime \prime} \sigma^{\prime \prime} ; S_{z}^{\prime \prime} / \psi_{k}\right\rangle\right]
\end{aligned}
$$

The summations over $m$ and $(m+1)$ on the R. H. S. of equation (Al.1) correspond to the possible combinations of electrons 
and holes in the intermediate states, similar to situation we had in considering one to three particle scattering equations in the text. The first term, as we mentioned above does not involve any sum over electron + hole combinations in the intermediate states for there is one and only one way in which a electron thole pair can be created from this state and is specified by our final $m$ electron state. This corresponds to the situation with single particle intermediate states in single particle to three particle scattering equations.

As before, the neglect of $(2 \mathrm{~m}+3)$ particle contributions in single particle to $(2 m+1)$ particle scattering equations of the form (A. 1.) shall provide a closed system of equations for $(2 \mathrm{~m}+1)$ particle projections. We can again solve this system by our summation technique and obtain the $(2 m+1)$ particle projection sums in terms of the single $(2 m-1)$ particle projections.

This procedure is in principle true for any $\mathrm{m}$. Thus, if we put $m=N$, the total number of electrons, we shall again have equations of the form (Al. 1) with one very critical difference. We shall no longer have intermediate states with $(N+1)$ electrons, for there are only $\mathrm{N}$ electrons in the system. Thus, in principle, one can obtain the $(2 \mathrm{~N}+1)$ particle projection sums in terms of the single $(2 N-1)$ particle projection sum occurring in the highest order equations of the form, 
$-165-$

$$
\begin{aligned}
& \left\langle\phi_{N-N}, N, k^{\prime} r^{\prime}, s_{Z}^{\prime} \mid \psi_{k}\right\rangle \\
& =\left[E_{k}-\left\{\left(\varepsilon_{0}+\epsilon_{k^{\prime}}+\epsilon_{k_{1}}+\cdots \cdot+\epsilon_{k_{N}}\right)-\left(\epsilon_{k_{\beta_{1}}}+\cdots+\epsilon_{k_{\beta_{N}}}\right)\right\}\right]^{-1} \\
& \cdot\left\{\sum_{k^{\prime \prime} \sigma^{\prime \prime}} \sum_{S_{z}^{\prime \prime}}\left\langle\phi_{N-(N-1)},(N-1), k^{\prime \prime} \sigma^{\prime \prime} ; s_{z}^{\prime \prime} \mid \psi_{k}\right\rangle\right. \\
& \left.+\sum_{k^{n} \sigma^{\prime \prime}} \sum_{s_{z}^{\prime \prime}} \sum_{N}\left\langle\phi_{N-N,} N, k^{n} \sigma^{\prime \prime} ; s_{Z}^{\prime \prime} \mid \psi_{K}\right\rangle\right\} \cdot(-J / N)
\end{aligned}
$$

(A1.2)

Knowing (2 $\mathrm{N}+1)$ particle projection sums, one can eliminate them from the scattering equation for $(2 \mathrm{~N}-1)$ particle projections which would involve $(2 \mathrm{~N}-3),(2 \mathrm{~N}-1)$ and $(2 \mathrm{~N}+1)$ particle projection sums. This shall leave us with equations involving $(2 \mathrm{~N}-3)$ and $(2 \mathrm{~N}-1)$ particle projection sums. This reduction technique can in principle be continued until we obtain the exact contribution of the three particle projection sums involved in our exact single particle to single particle scattering equations, (IV. 18) and (IV. 19), in terms of the single particle projections $\left\langle\phi_{N}, k^{\prime} \varphi ; s_{z} \mid \psi_{k}\right\rangle$ and $\left\langle\phi_{N}, k^{\prime} t ;\left(s_{z}+v\left|\psi_{k}\right\rangle\right.\right.$. This proves our ansatz. 


\section{APPENDIX 2}

We evaluate the integral $\chi(\omega, \beta)$ in this appendix. Let us consider the following integral,

$$
I(x)=\int_{-\infty}^{D} \frac{f\left(\beta x^{\prime}\right)-1 / 2}{x-x^{\prime}+i \eta} d x
$$

(A2. 1)

where $f\left(\beta x^{\prime}\right)$ is the fermi function. Then,

$$
I(x+2 \pi i / \beta)=\int_{-D}^{D} \frac{\left\{f\left(\beta x^{\prime}\right)-1 / 2\right\} d x^{\prime}}{x-x^{\prime}+2 \pi i / \beta+i \eta}
$$

(A2.2)

Putting $x^{\prime \prime}=\left(x^{\prime}-2 \pi i / \beta\right)$ and using the periodicity of the fermi function in the imaginary direction.

$$
\left\{e^{\beta x^{\prime}}+1\right\}^{-1}=\left\{e^{\beta\left(x^{\prime}+2 n \pi i / \beta\right)}+1\right\}^{-1}
$$

we find

$$
I(x+2 \pi i / \beta)=\int_{-D-2 \pi i / \beta}^{D-2 \pi i / \beta} d x^{\prime \prime}\left\{\frac{f\left(\beta x^{\prime \prime}\right)-1 / 2}{x-x^{\prime \prime}+i \eta}\right\}
$$


We now deform the contour indicated by (A2.3) and shown in Fig. (15) by the dashed line to one shown by the solid line. However, in doing so we pick up a contribution from one of the poles of the fermi function, which all lie on the imaginary axis

- Thus we obtain,

$$
\begin{aligned}
I(x+2 \pi i / \beta) & =I(x)-\frac{2 \pi i / \beta}{x+\pi i / \beta} \\
& +\int_{-D-2 \pi i / \beta}^{D} d x^{\prime \prime} \frac{f\left(\beta x^{\prime \prime}\right)-1 / 2}{x-x^{\prime \prime}+i \eta} \\
& +\int_{D}^{J-2 \pi i / \beta} d x^{\prime \prime} \frac{f\left(\beta x^{\prime \prime}\right)-1 / 2}{x-x^{\prime \prime}+i \eta}
\end{aligned}
$$

At this point, to evaluate the integrals over the vertical parts of the solid contour, we assume that $k_{B} T<<$, replace $\left(f-\frac{1}{2}\right)$ by $\pm \frac{1}{2}$ in the first and second integrands respectively and obtain,

$$
\begin{aligned}
I(x+2 \pi i / \beta) & =I(x)-\frac{2 \pi i / \beta}{x+\pi i / \beta} \\
& +(1 / 2) \ln \left\{\frac{D^{2}-(x+2 \pi i / \beta)^{2}}{D^{2}-x^{2}}\right\}
\end{aligned}
$$


Next, we make use of the Digamma function, defined (to within a constant) by the recurrence formula, (43)

$$
\psi(z+1)=\psi(z)+1 / z
$$

We notice that equation (A2.5) is a similar recurrence formula,

$$
\begin{gathered}
{\left[-I(x+2 \pi i / \beta)+(1 / 2) \ln \left\{\frac{(x+2 \pi i / \beta)^{2}-D^{2}}{(2 \pi i / \beta)^{2}}\right\}\right]} \\
=\left[-I(x)+(1 / 2) \ln \left\{\frac{x^{2}-D^{2}}{(2 \pi i / \beta)^{2}}\right\}\right] \\
+\left\{1 / 2+\frac{x}{2 \pi i / \beta}\right\}^{-1}
\end{gathered}
$$

(A2.6)

Examination of (A2.6) and the asymptotic form ${ }^{(43)}$ of the digamma function $\psi(1 / 2+x \beta / 2 \pi i)$, we find that we have chosen the constant of separation $(2 \pi i / \beta)^{2}$. Thus, each of the terms in square brackets in (A2.6) is a digamma function and we have

$$
\begin{aligned}
I(x)= & {\left[\frac{1}{2} \ln \left\{\frac{x^{2}-D^{2}}{(2 \pi i / \beta)^{2}}\right\}\right.} \\
& -\psi(1 / 2+\beta x / 2 \pi i)]
\end{aligned}
$$


$-169-$

Equation (A2. 7) is essentially exact. It describes the end point singularities correctly within terms of order exp. (- $\beta D)$. Since $D>>k_{B} T$, our assertion is essentially correct.

Now we find that the integral of interest to us is given by,

$$
\chi(\omega, \beta)=\rho_{0}\left(\epsilon_{F}\right) \int_{-0}^{D} \frac{\tanh \left(\beta x^{\prime} / 2\right)}{\omega-x^{\prime}+i \eta} d x^{\prime}
$$

Since,

$$
\tanh \left(\beta x^{\prime} / 2\right)=1-2 f\left(\beta x^{\prime}\right)
$$

we have,

$$
\begin{aligned}
X(\omega, \beta) & =-2 \rho_{0}\left(\epsilon_{F}\right) \int_{-D}^{D} \frac{f\left(\beta x^{\prime}\right)-1 / 2}{\omega-x^{\prime}+i \eta} \\
& =-2 \rho_{0}\left(\epsilon_{F}\right) I(\omega, \beta)
\end{aligned}
$$

Thus,

$$
X(\omega, \beta)=-2 \rho_{0}\left(\epsilon_{F}\right)\left[\frac{1}{2} \ln \frac{\omega^{2}-D^{2}}{(2 \pi i / \beta)^{2}}-\psi\left(\frac{1}{2}+\frac{\omega}{2 \pi i / \beta}\right)\right]
$$

(A2. 9) 


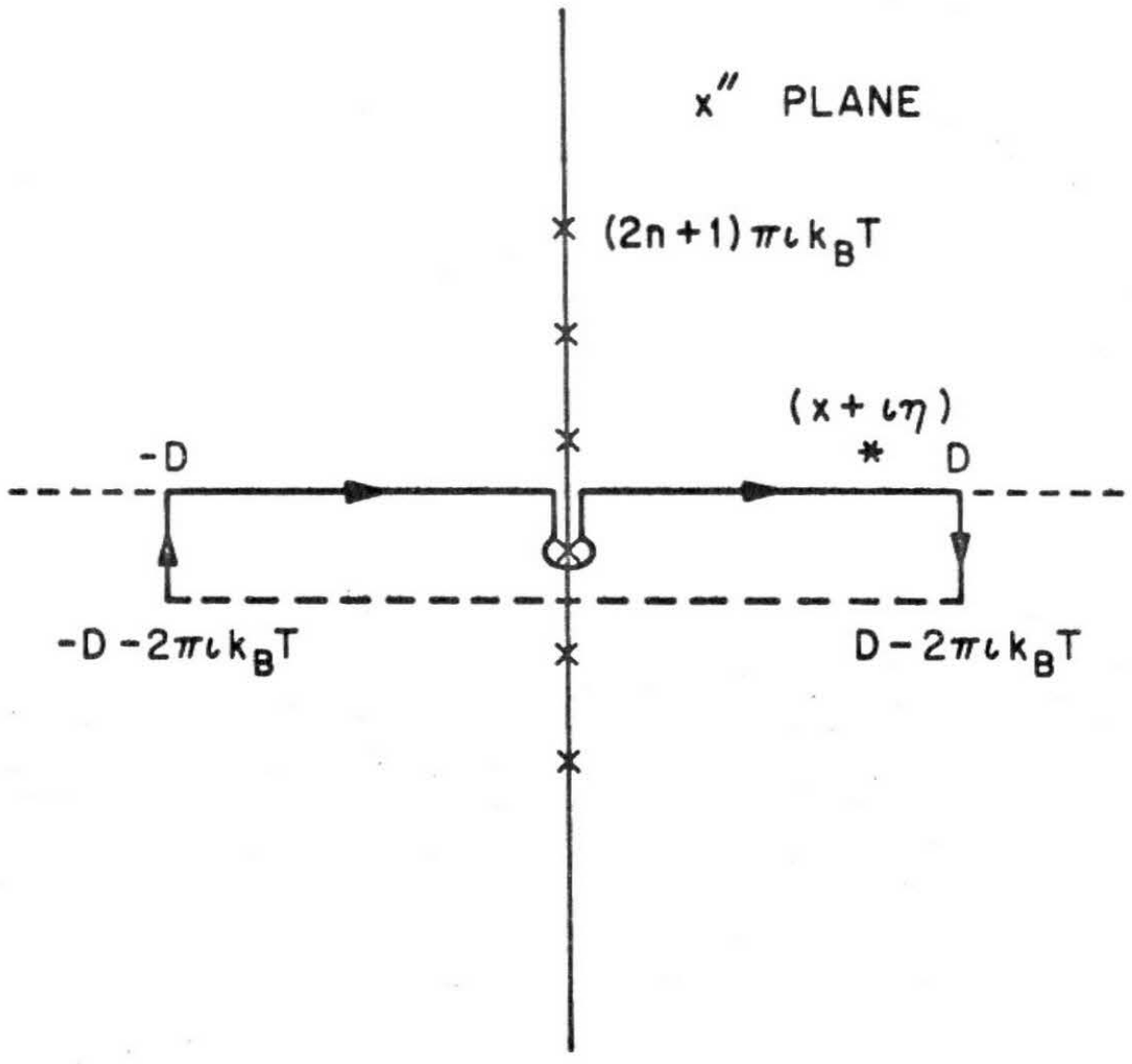

Fig. 15 Contour of integration for (A3), dashed line and the deformed contour, solid line. The $X$ 's are the poles of the integrand. 


\section{REFERENCES}

1. Friedel, J. Can. J. Phys. 34, 1190 (1956).

2. Anderson, P. W. Phys. Rev. 124, 41 (1961)

3. Wolff, P. A. Phys, Rev. 124, 1030 (1961).

4. Anderson, P. W. Les Horeches Ecole d'ete' 1967, The Many Body Problem, Ed. R. Balian and C. DeWitt, New York, Gordon and Breach, 1968.

5. Kondo, J. Solid State Physics, Ed. F. Seitz and D. Turnbull, New York; Academic Press 1969, Vol. 23.

6. Vandenberg, G. J. Progress in Low Temp. Phys., Ed. C. J. Gorter, Page 194, Vol. IV, North Holland Publ., Amsterdam, (1964).

7. Daybell, M. D. and Steyert, W. A. Rev. Mod. Phys. 40, 380 (1968).

8. Heeger, A. Solid State Physics, Ed. F. Seitz and D. Turnbull, New York, Academic Press, 1969, Vol. 23.

9. Kondo, J. Prog. Theo. Phys. (Kyoto) 32, 37 (1964).

10. Nagaoka, Y. Phys. Rev. 130, A1112 (1965).

11. Zubarev, D. N. Soviet Phys. Usph. (Eng. Trans.) $\underline{3}$, $320(1960)$.

12. Bloomfield, P. E. and Hamman, D. R. Phys. Rev. 164, 856 (1967).

13. Zittarz, J. and Mueller-Hartman, E. Z. Physik 212, 380 (1968). 
14. Suhl, H. Phys. Rev. 130 , A515 (1965); Physics 2, 39 (1965); Phys. Rev. 141, 483 (1966).

15. Suhl, H., and Wong D., Physics 3, 17 (1967).

16. Kondo, J., Prog. Theo. Phys. (Kyoto) 40, 695 (1968).

17. Silverstein, S. D., and Duke, C. B., Phys. Rev. 161, 456 (1967).

18. Brenig, W., and Gotze, W., Z. Physik, 217, 188 (1968).

19. Abrikosov, A. A., Physics 2, 5 (1965).

20. Doinach, S., Phys. Rev. 144, 302 (1966).

21. Applebaum J, and Kondo, J., Phys. Rev. 170, 542 (1968).

22. Yosida, K., Phys. Rev. 147, 223 (1966).

23. Cooper, L. N., Phys. Rev. 104, 1189 (1956).

24. Kondo, J., Prog. Theo. Phys. (Kyoto) 34, 204 (1965).

25. Takano, F., and Ogawa, T., Prog. Theo. Phys. (Kyoto) 35, 343 (1966).

26. Gorkov, L. P., Soviet Phys. JETP (Eng. Trans.) 7, 305 (1958).

27. Abrikosov, Gorkov and Dzyaloshienskii., Quantum Field Theoretical Methods in Statistical Physics, Pergamon Press (1965).

28. Zener, C. Phys. Rev. 21, 440 (1951).

29. Kasuya, T., Prog. Theo. Phys. (Kyoto) 16, 45 (1956).

30. Schrieffer, J. R., and Wolff, P. A., Phys. Rev. 149, $491(1966)$. 
31. Coglin, B., and Schrieffer, J. R., Phys. Rev. 125, 247 (1969).

32. Schweber, S. S., An Introduction to Relativistic Field Theory; Harper and Row, New York, (1961).

33. Barton, G. Dispersion Techniques in Field Theory, W. A. Benjamin Inc., New York (1965) Ch. 2.

34. Frøhlich, H., and Nabarro, F. R. N., Proc. Roy. Soc. A175, 382 (1940).

35. Ruderman, M. A., and Kittel, C., Phys. Rev. 96, 99 (1954).

36. Yosida, K., Phys. Rev. 106, 893 (1957).

37. Landshoff, P. V., in Rendiconti Scoula Inter nationale di Fisica "Enrico Fermi", course XXIX, Dispersion Relations and their connection with casuality, Page 137, Academic Press, New York, (1964).

38. Gell-Mann, M., and Goldberger, M. L., Phys. Rev. 91, 398 (1953).

39. Van Hove, L., Phys. Rev. 89, 1189 (1953).

40. Muskhelishvilli, N. I., Singular Integral Equations; translated by J. R. M. Raddock (P. Nordoff Ltd., Groningen, The Netherland s 1953), p. 229.

41. Luttinger, J. M., Phys. Rev. 121, 942 (1961).

42. Nozieres, P., Interacting Fermi Systems; Translated by D. Hone, (W. A. Benjamin, Inc., New York, 1964). Ch. 4. 
43. Abramowitz, M. in Handbook of Mathematical Functions; Edited by M. Abramowitz and I. A. Stegun, (Dover Publications, Inc., New York, 1965) Page 258, Eq. (6-3-5).

44. Schrieffer, J. R., J. Appl. Phys. 38, 1143 (1967).

45. Heeger, A. J. et al., Phys. Rev. 172, 302 (1968).

46. Mott, N. F., and Hones, H., in Theory of the Properties of Metals and Alloys; Dover Publications Inc., New York (1958), Ch. 6 .

47. Franck, J. P., Manchester, F. D., and Martin, D. L., Proc. Roy. Soc. (London) A263, 499 (1961).

48. Brock, J. C. F. et al., Solid State Comm. , 1139 (1970). 49. Star, W. M. et al., J. Appl. Phys. 41, 1152 (1970). 50. Cottet, H. et al., Helve Phys. Acta. 41, 755 (1968). 